\title{
On logarithmic extension of overconvergent isocrystals
}

\author{
Atsushi Shiho *
}

\begin{abstract}
In this paper, we establish a criterion for an overconvergent isocrystal on a smooth variety over a field of characteristic $p>0$ to extend logarithmically to its smooth compactification whose complement is a strict normal crossing divisor. This is a generalization of a result of Kedlaya, who treated the case of unipotent monodromy. Our result is regarded as a $p$-adic analogue of the theory of canonical extension of regular singular integrable connections on smooth varieties of characteristic 0 .
\end{abstract}

\section{Contents}

1 Log- $\nabla$-modules and $\Sigma$-unipotence

$2 \quad$ S-unipotence, generization and log-convergence $\quad 19$

3 Monodromy of isocrystals and logarithmic extension 35

\section{Introduction}

Let $K$ be a complete discrete valuation field of mixed characteristic $(0, p)$ with ring of integers $O_{K}$ and residue field $k$, and let $X \hookrightarrow \bar{X}$ be an open immersion of smooth $k$-varieties such that $Z=\bar{X}-X$ is a simple normal crossing divisor. Denote the log structure on $\bar{X}$ associated to $Z$ by $M$. The purpose of this paper is to give a criterion for an overconvergent isocrystal on $(X, \bar{X}) / K$ to extend to an isocrystal on log convergent site $\left((\bar{X}, M) / O_{K}\right)_{\text {conv }}$. This criterion is established by Kedlaya in [10] in the case of unipotent monodromy. In this paper, we generalize his result to the case of arbitrary monodromy (although we need certain ' $p$-adic non-Liouvilleness assumption'.)

${ }^{*}$ Graduate School of Mathematical Sciences, University of Tokyo, 3-8-1 Komaba, Meguro-ku, Tokyo 153-8914, JAPAN. E-mail address: shiho@ms.u-tokyo.ac.jp Mathematics Subject Classification (2000): 12H25 (primary), 14F30 (secondary) 
Our result can be regarded as a narutal $p$-adic analogue of the theory of canonical extension of regular singular integrable connections on algebraic varieties developped in [8]. So first we give a brief review of this theory.

First let us consider the local situation. Let $K$ be a discrete valuation field of equal characterisic 0 and denote the ring of integers by $O_{K}$. Let us fix a norm $|\cdot|$ corresponding to the valuation of $K$ and fix a uniformizer $t$ of $O_{K}$. Assume we are given a derivation $d: K \longrightarrow K d t=K \operatorname{dlog} t$ on $K$. Then we can define the notion of a connection $\nabla: E \longrightarrow E \operatorname{dlog} t$ on a finite dimensional vector space $E$ over $K$. Let us assume given such $(E, \nabla)$ and let $\partial$ be the composite map $E \stackrel{\nabla}{\longrightarrow} E \operatorname{dlog} t \stackrel{\Rightarrow}{\longrightarrow} E$, where the second map sends $e \operatorname{dlog} t$ to $e(e \in E)$. Then $(E, \nabla)$ is called regular singular if the spectral norm $|\partial|_{\mathrm{sp}}:=\lim _{n \rightarrow \infty}\left|\partial^{n}\right|^{1 / n}$ (where $\left|\partial^{n}\right|$ denotes the operator norm of $\left.\partial^{n}\right)$ of $\partial$ is equal to $1\left(=|d|_{\mathrm{sp}}\right)([8$, II 1.9, 1.11]). It is known ([8, II 1.12]) that $(E, \nabla)$ is regular singular if and only if it comes from a $\log$ connection $\nabla: E_{0} \longrightarrow E_{0} \mathrm{~d} \log t$ on a finitely generated free $O_{K}$-module $E_{0}$, that is, it extends to $O_{K}$ logarithmically.

In global situation, we can define the notion of regular singularity in the following way: Let $j: X \hookrightarrow \bar{X}$ be an open immersion of smooth $\mathbb{C}$-varieties such that $Z=$ $\bar{X}-X$ is a simple normal crossing divisor. Let us denote the log structure on $\bar{X}$ associated to $Z$ by $M$. Then a locally free $\mathcal{O}_{X}$-module $E$ endowed with an integrable connection $\nabla: E \longrightarrow E \otimes \Omega_{X / \mathbb{C}}^{1}$ is called regular singular if, for any generic point $\eta$ of $Z$, the restriction of $(E, \nabla)$ to $\left(j_{*} \mathcal{O}_{X}\right)_{\eta}(=\mathbb{C}(X)$ endowed with the discrete valuation associated to $\eta)$ is regular singular ( $[8$, II $4.2,4.4])$. Let us assume given such $(E, \nabla)$. Then, if $(E, \nabla)$ is unipotent (that is, the monodromy representation associated to $(E, \nabla)$ is unipotent along each irreducible component of $Z)$, it extends uniquely to an integrable log connection on $(\bar{X}, M)$ which has nilpotent residues ([8, II 5.2]). In general case, if we fix a section $\tau: \mathbb{C} / \mathbb{Z} \longrightarrow \mathbb{C}$ of the canonical projection, we can extend $(E, \nabla)$ uniquely to an integrable $\log$ connection on $(\bar{X}, M)$ whose exponents along each irreducible component of $Z$ are in $\tau(\mathbb{C} / \mathbb{Z})([\mathbb{8}$, II 5.4]).

Now let us return to the $p$-adic situation. First we consider the local situation. Let $K$ be a complete non-Archimedean valued field with ring of integers $O_{K}$ and residue field $k$ of characteristic $p>0$. For $\lambda \in\{0\} \cup p^{\mathbb{Q}<0}$, let $A_{K}^{1}[\lambda, 1)$ be the rigid analytic annulus with radius in $[\lambda, 1)$ over $K$. Consider a locally free module $E$ of finite rank on $A_{K}^{1}[\lambda, 1)$ endowed with a connection $\nabla: E \longrightarrow E d t$ (where $t$ is the coordinate of the annulus $A_{K}^{1}[\lambda, 1)$ ) and define $\partial$ by the composite map $E \stackrel{\nabla}{\longrightarrow} E d t \stackrel{=}{\longrightarrow} E$, where the second map sends $e d t$ to $e(e \in E)$. For $\rho \in[\lambda, 1) \cap p^{\mathbb{Q}}$, Let us denote the spectral norm on $\Gamma\left(A_{K}^{1}[\rho, \rho], \mathcal{O}\right)$ by $|\cdot|_{\rho}$. We call that $(E, \nabla)$ satisfies the Robba condition if the spectral norm $|\partial|_{\mathrm{sp}, \rho}=\lim _{n \rightarrow \infty}\left|\partial^{n}\right|_{\rho}^{1 / n}$ of $\partial$ at $\rho$ is equal to the spectral norm $|d|_{\mathrm{sp}, \rho}$ of $d\left(=\right.$ derivation on $\left.\mathcal{O}_{A_{K}^{1}[\lambda, 1)}\right)$ at $\rho$ for any $\rho \in[\lambda, 1) \cap p^{\mathbb{Q}}$. (See $[7$, p.136, 11.1].) For such $(E, \nabla)$ of $\operatorname{rank} \mu$, one can define the notion of exponent in the sense of Christol-Mebkhout, which is an element of $\mathbb{Z}_{p}^{\mu}$ modulo certain equivalence relation (see [7, 11.5] for detail). The Robba condition is considered as a $p$-adic analogue of the notion of regular singularity. Indeed, it is 
known $([6,6-2.6],[7,12.1]$ plus some calculation) that if $(E, \nabla)$ satisfies the Robba condition and its exponent (in the sense of Christol-Mebkhout) has p-adically nonLiouville difference (see [7, 10.8] for definition), $(E, \nabla)$ extends to a locally free module with log connection on $A_{K}^{1}[0,1)$.

Let us consider now the global situation: Let $K, O_{K}, k$ be as above and assume that $K$ is discretely valued. Let $X \hookrightarrow \bar{X}$ be an open immersion of smooth $k$-varieties such that $Z=\bar{X}-X=\bigcup_{i=1}^{r} Z_{i}$ is a simple normal crossing divisor. Let us denote the $\log$ structure on $\bar{X}$ associated to $Z$ by $M$. We will define, for an overconvergent isocrystal $\mathcal{E}$ on $(X, \bar{X}) / K$, the notion of the Robba condition. (See Definition 3.13 for detail.) Roughly speaking, we call that $\mathcal{E}$ satisfies the Robba condition if, for any generic point $\eta$ of $Z$, we can take certain annulus $A_{L}^{1}[\lambda, 1$ ) over $\operatorname{Spm} L$ (which is a field defined by using certain lift around $\eta$ ) such that the connection on $A_{L}^{1}[\lambda, 1)$ induced by $\mathcal{E}$ satisfies the Robba condition. We call $\mathcal{E}$ is (NLD) when the exponents (in the sense of Christol-Mebkhout) of the connections on the annuli have $p$-adically non-Liouville difference for any $\eta$. For an overconvergent isocrystal $\mathcal{E}$ on $(X, \bar{X}) / K$ satisfying the Robba condition which is (NLD), we can define the set $\bar{\Sigma}_{\mathcal{E}}$ of exponents of $\mathcal{E}$, which is a subset of $\left(\mathbb{Z}_{p} / \mathbb{Z}\right)^{r}$. (See $\S 3$ for detail.)

With this terminology, we can state our main result as follows: Let $\mathcal{E}$ be an overconvergent isocrystal on $(X, \bar{X}) / K$ satisfying the Robba condition which is (NLD). Then, if we fix a section $\tau:\left(\mathbb{Z}_{p} / \mathbb{Z}\right)^{r} \longrightarrow \mathbb{Z}_{p}^{r}$ of the canonical projection, we can extend $\mathcal{E}$ uniquely to an isocrystal on $\log$ convergent site $\left((\bar{X}, M) / O_{K}\right)_{\text {conv }}$ whose exponents along $Z$ are contained in $\tau\left(\bar{\Sigma}_{\mathcal{E}}\right)$. This is a $p$-adic analogue of the logarithmic extension of integrable connections which we explained above. We would like to remark here that this result is proved by Kedlaya ([10]) in the case where $\mathcal{E}$ has unipotent monodromy (the case $\bar{\Sigma}_{\mathcal{E}}=\{0\}$ and $\tau(0)=0$ ).

The strategy of the proof is basically the same as that in [10], which we briefly explain here. By the assumption of the Robba condition and the condition (NLD), $\mathcal{E}$ extends logarithmically to the discs $A_{L}^{1}[0,1)$ at each generic point of $Z$. First we prove that this condition implies the log-extendability of $\mathcal{E}$ to relative disc over ] $Z^{0}$ [, where $Z^{0}$ is the smooth locus of $Z$ and ] · [ denotes certain rigid analytic space defined by using some local lift of $Z^{0}$. We prove this by using the proposition called 'generizarion' (see Proposition 2.4). Next we prove the log-extendability of $\mathcal{E}$ to certain relative disc over a strict neighborhood of $] Z^{0}[$ in $] Z[$, by using the proposition called 'overconvergent generization' (see Proposition 2.7). Then we extend $\mathcal{E}$ logarithmically to the boundary step by step, along each irreducible component of $Z$.

Let us explain the content of each section. In Section 1, we prove basic properties on log- $\nabla$-modules on rigid spaces. We introduce the notion of $\log -\nabla$-modules with exponents in $\Sigma$ and the notion of $\Sigma$-unipotent $\log$ - $\nabla$-modules $\left(\Sigma \subseteq \bar{K}^{r}\right.$ for some $r$ ), which are the generalization of $\log -\nabla$-modules with nilpotent residues and unipotent $\log -\nabla$-modules introduced in [10, §3]. In Section 2, we prove the three key propositions which we need for the proof of our main result. The first one, called generization, asserts that the property of $\Sigma$-unipotence is 'generic on the 
base'. The second one, called overconvergent generization, asserts that the property of $\Sigma$-unipotence can be extended 'overconvergently on the base'. The third one asserts that, under the condition of log-convergence, the property of 'having exponents in $\Sigma$ ' implies the $\Sigma$-unipotence. They are also generalization of the corresponding results of Kedlaya in [10], but we would like to point out here that our results in this section are slightly different from those in Kedlaya partly because Kedlaya's argument in [10,3.5.3] and [10, 3.6.2] seems to contain an error 1 and partly because of the technical reason. In Section 3, we prove the main result of this paper, using the results in the previous section. We introduce the notion of an isocrystal on log convergent site with exponents in $\Sigma$ and the notion of an overconvergent isocrystal having $\Sigma$-unipotent monodromy. Also we introduce the notion of an overconvergent isocrystal satisfying the Robba condition and the property (NLD), and check that an overconvergent isocrystal satisfying the Robba condition and the property (NLD) has $\Sigma$-unipotent monodromy (for certain $\Sigma$ ), by using generization. Then we prove that, under some condition on $\Sigma$, an overconvergent isocrystal having $\Sigma$-unipotent monodromy can be extended uniquely to an isocrystal on log convergent site with exponents in $\Sigma$, by using the three key propositions proved in the previous section.

A part of this work was done during the author's stay at Université de Rennes I. The author would like to thank to Pierre Berthelot for giving me an opportunity to stay there and to the members there for the hospitality. The author is partly supported by Grant-in-Aid for Young Scientists (B), the Ministry of Education, Culture, Sports, Science and Technology of Japan and JSPS Core-to-Core program 18005 whose representative is Makoto Matsumoto.

\section{Convention}

(1) Throughout this paper, $k$ is a field of characteristic $p>0$ and a $k$-variety means a reduced separated scheme of finite type over $k$.

(2) Throughout this paper, $K$ is a field of characteristic 0 complete with respect to the non-Archimedean absolute value $|\cdot|: K \longrightarrow \mathbb{R}_{\geq 0}$ with residue field $k$ of characteristic $p>0$. Let us put $\Gamma^{*}:=\sqrt{\left|K^{\times}\right|} \cup\{0\}$ and let $O_{K}$ be the the ring of integers of $K$. In Section 3, we will assume moreover that the absolute value $|\cdot|$ is discretely valued. We denote the algebraic closure of $K$ by $\bar{K}$. For a $p$-adic formal scheme $P$ topologically of finite type over $O_{K}$, we denote the associated rigid space by $P_{K}$.

(3) We use freely the notion concerning isocrystals on log convergent site and overconvergent isocrystals. For the former, see [14], [15] and [10, §6]. For the latter, see [4] and [10, §2].

\footnotetext{
${ }^{1}$ After putting the first version of this paper to ArXiv, Kedlaya informed me how to correct the errors in the paper [10, which is different from the argument in this paper. His correction is written in [11, Appendix A].
} 


\section{$1 \quad$ Log- $\nabla$-modules and $\Sigma$-unipotence}

In this section, first we recall the notion of $\left(\log _{-}\right) \nabla$-modules and the residues of $\log -\nabla$-modules, following [10, 2.3]. Then we recall the notion of exponents of the residues, following [2] and introduce the notion of $\Sigma$-unipotence for log- $\nabla$-modules for a subset $\Sigma$ of $\bar{K}^{r}$ (for some $r$ ): It is a generalization of the notion of unipotence for $\log -\nabla$-modules introduced in [10, 3.2.5]. After that, we prove some basic properties on $\log -\nabla$-modules with exponents in $\Sigma$ and $\Sigma$-unipotent $\log -\nabla$-modules, which are generalization of the results in [10, 3.2].

First let us recall the definition of $\left(\log _{-}\right) \nabla$-modules. For a rigid space $X$ over $K$, let $\Omega_{X / K}^{1}$ be the sheaf of continuous differentials on $X$ over $K$ and for a morphism $f: X \longrightarrow Y$ of rigid spaces over $K$, let us put $\Omega_{X / Y}^{1}:=\Omega_{X / K}^{1} / f^{*} \Omega_{Y / K}^{1}$.

Definition 1.1 ([10, 2.3.4]). Let $f: X \longrightarrow Y$ be a morphism of rigid spaces over $K$. A $\nabla$-module on $X$ relative to $Y$ is a coherent $\mathcal{O}_{X}$-module $E$ endowed with an integrable $f^{-1} \mathcal{O}_{Y}$-linear connection $\nabla: E \longrightarrow E \otimes_{\mathcal{O}_{X}} \Omega_{X / Y}^{1}$. In the case $Y=\operatorname{Spm} K$, we omit the term 'relative to $Y$ '.

It is known $([4,2.2 .3])$ that when $X$ is a smooth rigid space, any $\nabla$-module on $X$ (relative to $\operatorname{Spm} K$ ) is automatically locally free.

Next, when we are given a morphism $f: X \longrightarrow Y$ of rigid spaces over $K$ and let $x_{1}, \ldots, x_{r}$ be elements in $\Gamma\left(X, \mathcal{O}_{X}\right)$. Then we define the module of logarithmic differentials $\omega_{X / Y}^{1}$ (denoted by $\Omega_{X / Y}^{1, \log }$ in [10]) by

$$
\omega_{X / Y}^{1}:=\left(\Omega_{X / Y}^{1} \oplus \bigoplus_{i=1}^{r} \mathcal{O}_{X} \cdot \operatorname{dlog} x_{1}\right) / N,
$$

where $N$ is the sheaf locally generated by $\left(d x_{i}, 0\right)-\left(0, x_{i} \operatorname{dlog} x_{i}\right)(1 \leq i \leq r)$.

Definition $1.2([10,2.3 .7])$. Let $f: X \longrightarrow Y$ be a morphism of rigid spaces over $K$ and let $x_{1}, \ldots, x_{r}$ be elements in $\Gamma\left(X, \mathcal{O}_{X}\right)$. Then a log- $\nabla$-module on $X$ with respect to $x_{1}, \ldots, x_{r}$ relative to $Y$ is a locally free $\mathcal{O}_{X}$-module $E$ endowed with an integrable $f^{-1} \mathcal{O}_{Y}$-linear $\log$ connection $\nabla: E \longrightarrow E \otimes_{\mathcal{O}_{X}} \omega_{X / Y}^{1}$. In the case $Y=\operatorname{Spm} K$, we omit the term 'relative to $Y$ '.

Note that, in the case of $\log -\nabla$-modules, the local freeness is not automatic even in the case $Y=\operatorname{Spm} K$. So it is built in the definition.

Let $f: X \longrightarrow Y, x_{1}, \ldots, x_{r}$ be as in Definition 1.2 and let $(E, \nabla)$ be a $\log -\nabla$ module on $X$ with respect to $x_{1}, \ldots, x_{r}$ relative to $Y$. For $1 \leq i \leq r$, let us put $D_{i}:=\left\{x_{i}=0\right\} \subseteq X$ and $M_{i}:=\operatorname{Im}\left(\Omega_{X / Y}^{1} \oplus \bigoplus_{j \neq i} \mathcal{O}_{X} \mathrm{~d} \log x_{j} \longrightarrow \omega_{X / Y}^{1}\right)$. Then the composite map

$$
E \stackrel{\nabla}{\longrightarrow} E \otimes_{\mathcal{O}_{X}} \omega_{X / Y}^{1} \longrightarrow E \otimes_{\mathcal{O}_{X}}\left(\omega_{X / Y}^{1} / M_{i}\right) \cong E \otimes_{\mathcal{O}_{X}} \mathcal{O}_{D_{i}} \mathrm{~d} \log x_{i} \cong E \otimes_{\mathcal{O}_{X}} \mathcal{O}_{D_{i}}
$$

naturally induces an element $\operatorname{res}_{i}$ in $\operatorname{End}_{\mathcal{O}_{D_{i}}}\left(E \otimes_{\mathcal{O}_{X}} \mathcal{O}_{D_{i}}\right)$. We call it the residue of $(E, \nabla)$ along $D_{i}$. 
Keep the above notation and assume that $Y=\operatorname{Spm} K, X$ is a smooth affinoid rigid space and that the zero loci of $x_{i}$ are smooth and meet transversally. Then, by the argument of $[2,1.5 .3]$, we see that there exists a polynomial $P_{i}(x) \in K[x](1 \leq$ $i \leq r)$ satisfying $P_{i}\left(\operatorname{res}_{i}\right)=0$. Take $P_{i}(x)$ to be minimal and monic satisfying $P_{i}\left(\operatorname{res}_{i}\right)=0$. Then we call the roots of $P_{i}(x)$ (in $\left.\bar{K}\right)$ the exponents of $(E, \nabla)$ along $D_{i}$. When $X$ is not necessarily affinoid, the set of exponents of $(E, \nabla)$ is defined as the union of the sets of the exponents of $\left.(E, \nabla)\right|_{U}$ for affinoid admissible opens $U \subseteq X$.

Let $X$ be a smooth rigid space endowed with $x_{1}, \ldots, x_{r} \in \Gamma\left(X, \mathcal{O}_{X}\right)$ such that the zero loci of $x_{i}$ are smooth and meet transversally. For a subset $\Sigma=\prod_{i=1}^{r} \Sigma_{i}$ in $\bar{K}^{r}$ $\left(\Sigma_{i} \subseteq \bar{K}\right)$, we define the category $\mathrm{LNM}_{X, \Sigma}$ as the category of log- $\nabla$-modules on $X$ with respect to $x_{1}, \ldots, x_{r}$ such that all the exponents along $D_{i}$ are contained in $\Sigma_{i}$ $(1 \leq i \leq r)$.

As in [10], we call a subinterval $I \subseteq[0, \infty)$ aligned if any endpoint of $I$ at which it is closed is contained in $\Gamma^{*}$. We call a subinterval $I \subseteq[0, \infty)$ quasi-open when it is open at non-zero endpoints. For an aligned interval $I$, we define the rigid space $A_{K}^{n}(I)$ by $A_{K}^{n}(I):=\left\{\left(t_{1}, \ldots, t_{n}\right) \in \mathbb{A}_{K}^{n, \text { an }}|\forall i,| t_{i} \mid \in I\right\}$.

Following [10, 3.2.4], we use the following convention: For a smooth affinoid rigid space $X$ endowed with $x_{1}, \ldots, x_{r} \in \Gamma\left(X, \mathcal{O}_{X}\right)$ such that the zero loci of $x_{i}$ are smooth and meet transversally, we put $\omega_{X \times A_{K}^{n}[0,0] / K}^{1}:=\omega_{X / K}^{1} \oplus \bigoplus_{i=1}^{n} \mathcal{O}_{X} d \log t_{i}$, where dlog $t_{i}$ is the free generator 'corresponding to the $i$-th coordinate on $A_{K}^{n}[0,0]$ '. Using this, we can define the notion of a $\log -\nabla$-module $(E, \nabla)$ on $X \times A_{K}^{n}[0,0]$ with respect to $x_{1}, \ldots, x_{r}, t_{1}, \ldots, t_{n}$ and the notion of the residue, the exponents of $(E, \nabla)$ along $\left\{t_{i}=0\right\}$ in natural way: To give a $\log -\nabla$-module $(E, \nabla)$ on $X \times A_{K}^{n}[0,0]$ with respect to $x_{1}, \ldots, x_{r}, t_{1}, \ldots, t_{n}$ is equivalent to give a $\log -\nabla$-module $(E, \nabla)$ on $X$ with respect to $x_{1}, \ldots, x_{r}$ and commuting endomorphisms $\partial_{i}:=t_{i} \frac{\partial}{\partial t_{i}}$ of $(E, \nabla)(1 \leq i \leq n)$. Also, we can define the category $\operatorname{LNM}_{X \times A_{K}^{n}[0,0], \Sigma}$ for $\Sigma=\prod_{i=1}^{r+n} \Sigma_{i} \subseteq \bar{K}^{r+n}$ as above: A $\log -\nabla$-module $(E, \nabla)$ on $X \times A_{K}^{n}[0,0]$ with respect to $x_{1}, \ldots, x_{r}, t_{1}, \ldots, t_{n}$, regarded as a $\log -\nabla$-module $(E, \nabla)$ on $X$ with respect to $x_{1}, \ldots, x_{r}$ endowed with commuting endomorphisms $\partial_{i}:=t_{i} \frac{\partial}{\partial t_{i}}(1 \leq i \leq n)$, is in the category $\operatorname{LNM}_{X \times A_{K}^{n}[0,0], \Sigma}$ if and only if $(E, \nabla)$ is in $\operatorname{LNM}_{X, \prod_{i=1}^{r} \Sigma_{i}}$ and all the eigenvalues of $\partial_{i}$ (which are a priori contained in $\bar{K})$ are in $\Sigma_{r+i}(1 \leq i \leq n)$.

For an aligned interval $I \subseteq[0, \infty)$ and $\xi:=\left(\xi_{1}, \ldots, \xi_{n}\right) \in K^{n}$, we define the log$\nabla$-module $\left(M_{\xi}, \nabla_{M_{\xi}}\right)$ on $A_{K}^{n}(I)$ with respect to $t_{1}, \ldots, t_{n}$ (which are the coordinates) as the log- $\nabla$-module $\left(\mathcal{O}_{A_{K}^{n}(I)}, d+\sum_{i=1}^{n} \xi_{i} \operatorname{dlog} t_{i}\right)$. Now we introduce the notion of (potential) $\Sigma$-constance and (potential) $\Sigma$-unipotence for $\log -\nabla$-modules:

Definition 1.3 (cf. [10, 3.2.5]). Let $X$ be a smooth rigid space endowed with $x_{1}$, $\ldots, x_{r} \in \Gamma\left(X, \mathcal{O}_{X}\right)$ whose zero loci are smooth and meet transversally. Let $I \subseteq[0, \infty)$ be an aligned interval and fix $\Sigma:=\prod_{i=1}^{r+n} \Sigma_{i} \subseteq \bar{K}^{r+n}$.

(1) An object $(E, \nabla)$ in $\operatorname{LNM}_{X \times A_{K}^{n}(I), \Sigma}\left(\right.$ where $X \times A_{K}^{n}(I)$ is endowed with $x_{1}, \ldots, x_{r}$, 
$t_{1}, \ldots, t_{n}$, where $t_{i}$ 's are the coordinates in $\left.A_{K}^{n}(I)\right)$ is called $\Sigma$-constant if $(E, \nabla)$ has the form $\pi_{1}^{*}\left(F, \nabla_{F}\right) \otimes \pi_{2}^{*}\left(M_{\xi}, \nabla_{M_{\xi}}\right)$ for some $\left(F, \nabla_{F}\right) \in \operatorname{LNM}_{X, \prod_{i=1}^{r} \Sigma}$ and $\xi \in$ $\prod_{i=r+1}^{r+n}\left(\Sigma_{i} \cap K\right)$, where $\pi_{1}: X \times A_{K}^{n}(I) \longrightarrow X, \pi_{2}: X \times A_{K}^{n}(I) \longrightarrow A_{K}^{n}(I)$ denote the projections.

(2) An object $(E, \nabla)$ in $\operatorname{LNM}_{X \times A_{K}^{n}(I), \Sigma}$ is called $\Sigma$-unipotent if $(E, \nabla)$ admits a filtration

$$
0=E_{0} \subset E_{1} \subset \cdots \subset E_{m}=E
$$

by sub log- $\nabla$-modules whose successive quotients are $\Sigma$-constant $\log -\nabla$-modules.

(3) An object $(E, \nabla)$ in $\mathrm{LNM}_{X \times A_{K}^{n}(I), \Sigma}$ is called potentially $\Sigma$-constant (resp. potentially $\Sigma$-unipotent) if it is $\Sigma$-constant (resp. $\Sigma$-unipotent) after some finite extension of the field $K$.

We denote the category of $\Sigma$-unipotent (resp. potentially $\Sigma$-unipotent) $\log -\nabla$ modules on $X \times A_{K}^{n}(I)$ with respect to $x_{1}, \ldots, x_{r}, t_{1}, \ldots, t_{n}$ by $\operatorname{ULNM}_{X \times A_{K}^{n}(I), \Sigma}$ (resp. $\left.\mathrm{ULNM}_{X \times A_{K}^{n}(I), \Sigma}^{\prime}\right)$.

Remark 1.4. Let the notation be as above and assume that $r=0$ and that $I$ does not contain 0 . In this case, it is easy to see that the $\log -\nabla$-modules $M_{\xi}$ and $M_{\xi^{\prime}}\left(\xi, \xi^{\prime} \in \Sigma\right)$ are isomorphic if $\xi-\xi^{\prime}$ is contained in $\mathbb{Z}^{n}$. From this fact, it is easy to see that the notion of (potential) $\Sigma$-unipotence only depends on the image $\bar{\Sigma}$ of $\Sigma$ in $(\bar{K} / \mathbb{Z})^{n}$ in the following sense: An object $(E, \nabla)$ in $\operatorname{LNM}_{X \times A_{K}^{n}(I), \Sigma}$ is (potentially) $\Sigma$-unipotent if and only if it is $\tau(\bar{\Sigma})$-unipotent for some (or any) section $\tau:(\bar{K} / \mathbb{Z})^{n} \longrightarrow \bar{K}^{n}$ of the canonical projection $\bar{K}^{n} \longrightarrow(\bar{K} / \mathbb{Z})^{n}$. So, in this case, we will say also that $(E, \nabla)$ is (potentially) $\bar{\Sigma}$-unipotent, by abuse of terminology.

Following [10, 3.2.6], we introduce an important functor $\mathcal{U}_{I}$ :

Definition 1.5. Let $X, I, \Sigma$ be as in Definition 1.3. Then we define the functor $\mathcal{U}_{I}: \mathrm{LNM}_{X \times A_{K}^{n}[0,0], \Sigma} \longrightarrow \mathrm{LNM}_{X \times A_{K}^{n}(I), \Sigma}$ as follows: For an object in $\operatorname{LNM}_{X \times A_{K}^{n}[0,0], \Sigma}$ regarded as $\left((E, \nabla),\left\{\partial_{i}\right\}_{i=1}^{n}\right)\left((E, \nabla) \in \mathrm{LNM}_{X, \prod_{i=1}^{r} \Sigma_{i}}\right)$ is sent by $\mathcal{U}_{I}$ to $\pi^{*} E$ (where $\pi: X \times A_{K}^{n}(I) \longrightarrow X$ denotes the projection) endowed with the connection

$$
\mathbf{v} \mapsto \pi^{*} \nabla(\mathbf{v})+\sum_{i=1}^{n} \pi^{*}\left(\partial_{i}\right)(\mathbf{v}) \operatorname{dlog} t_{i}
$$

We have the following remark, which is parallel to [10, 3.2.7]:

Remark 1.6. Let the notation be as above.

(1) In the case $X=\operatorname{Spm} K(r=0)$ and $I=[0,0]$, a log- $\nabla$-module on $X \times A_{K}^{n}(I)$ is nothing but a finite dimensional $K$-vector space $E$ endowed with commuting endomorphisms $\partial_{i}(1 \leq i \leq n)$. It is (potentially) $\Sigma$-constant if and only if each $\partial_{i}$ is equal to the multiplication by $\xi_{i}$ for some $\xi_{i} \in \Sigma_{i} \cap K$. It is $\Sigma$ unipotent (resp. potentially $\Sigma$-unipotent) if and only if each $\partial_{i}$ has eigenvalues 
in $\Sigma_{i} \cap K$ (resp. $\Sigma_{i}$ ). So, in this case, $\Sigma$-constance is equivalent to potential $\Sigma$-constance and $\Sigma$-unipotence (resp. potential $\Sigma$-unipotence) is equivalent to being contained in the category $\mathrm{LNM}_{X \times A_{K}^{n}(I), \Sigma \cap K^{n}}\left(\right.$ resp. $\left.\operatorname{LNM}_{X \times A_{K}^{n}(I), \Sigma}\right)$.

(2) From the remark (1), we easily see that, in the case $X=\operatorname{Spm} K(r=0)$ and $I$ arbitrary, an object in the image of $\mathcal{U}_{I}$ is potentially $\Sigma$-unipotent and it is $\Sigma$-unipotent if $\Sigma \subseteq K^{n}$.

Before investigating the properties of $\log -\nabla$-modules with exponents in $\Sigma$ and (potentially) $\Sigma$-unipotent log- $\nabla$-modules, we consider several conditions on the set $\Sigma$. To do this, first we recall the notion of the type of a number in $\bar{K}$ and that of $p$-adic non-Liouvilleness:

Definition 1.7. (1) For $a \in \bar{K}$, define the type type $(a) \in[0,1]$ of a by the radius of convergence of the series $\sum_{s \in \mathbb{N}, \neq a} \frac{X^{s}}{a-s}$.

(2) $a \in \bar{K}$ is called $p$-adically non-Liouville if type $(a)=\operatorname{type}(-a)=1$ holds.

Definition 1.8. A set $\Sigma \subseteq \bar{K}$ is called (NID) (resp. (PTD), (NLD)) if, for any $\alpha, \beta \in \Sigma, \alpha-\beta$ is not a non-zero integer (resp. the type of $\alpha-\beta$ is positive, $\alpha-\beta$ is p-adically non-Liouville). A set $\Sigma:=\prod_{i=1}^{r} \Sigma_{i} \subseteq \bar{K}^{r}$ is called (NID) (resp. (PTD), (NLD)) if so is $\Sigma_{i}$ for all $1 \leq i \leq r$.

We say a set $\bar{\Sigma}:=\prod_{i=1}^{r} \bar{\Sigma}_{i} \subseteq(\bar{K} / \mathbb{Z})^{r}$ is (PTD) (resp. (NLD)) if, for some (or any) section $\tau:(\bar{K} / \mathbb{Z})^{r} \longrightarrow \bar{K}^{r}$ of the canonical projection, $\tau(\bar{\Sigma})$ is (PTD) (resp. $(\mathrm{NLD}))$. Note that $\tau(\bar{\Sigma})$ is automatically (NID).

Now we prove the analogue of [10, 3.2.8], which is a starting point of the study of log- $\nabla$-modules with exponents in $\Sigma$ :

Lemma 1.9. Let $X$ be a smooth rigid space endowed with $x_{1}, \ldots, x_{r} \in \Gamma\left(X, \mathcal{O}_{X}\right)$ whose zero loci are smooth and meet transversally. Let $\Sigma:=\prod_{i=1}^{r+s+1} \Sigma_{i}$ be a subset of $\bar{K}^{r+s}$ such that $\Sigma_{r+1}$ is (NID) and (PTD), let $a \in(0, \infty) \cap \Gamma^{*}$ and let $(E, \nabla)$ be an object in $\mathrm{LNM}_{X \times A_{K}^{1}[0, a] \times A_{K}^{s}[0,0], \Sigma}$ such that $\left.E\right|_{X \times\{0\}}$ is free. Then there exists an element $b \in(0, a] \cap \Gamma^{*}$ such that the restriction of $E$ to $X \times A_{K}^{1}[0, b] \times A_{K}^{s}[0,0]$ is in the essential image of $\mathcal{U}_{[0, b]}^{\prime}: \mathrm{LNM}_{X \times A_{K}^{1}[0,0] \times A_{K}^{s}[0,0], \Sigma} \longrightarrow \mathrm{LNM}_{X \times A_{K}^{1}[0, b] \times A_{K}^{s}[0,0], \Sigma}$, where $\mathcal{U}_{[0, b]}^{\prime}$ is the functor naturally induced by $\mathcal{U}_{[0, b]}: \operatorname{LNM}_{X \times A_{K}^{1}[0,0], \prod_{i=1}^{r+1} \Sigma_{i}} \longrightarrow$ $\mathrm{LNM}_{X \times A_{K}^{1}[0, b], \prod_{i=1}^{r+1} \Sigma_{i}}$.

Proof. We give a proof, following [10, 3.2.8]. For a commutative ring $A$ and $n \in \mathbb{N}$, we denote the set of $n \times n$ matrices with entries in $A$ by $\operatorname{Mat}_{n}(A)$. Also, we denote the coodinate of $A_{K}^{1}[0, a]$ by $t$ and denote the differential operator $t \frac{\partial}{\partial t}$ simply by $\partial$.

Let us take a basis $\mathbf{e}_{1}, \ldots, \mathbf{e}_{n}$ of $\left.E\right|_{X \times\{0\}}$. Then, as in the proof of [10, 3.2.8], we see that a lift of this basis (which we denote again by $\mathbf{e}_{1}, \ldots, \mathbf{e}_{n}$ ) forms a basis of $E$ on $X \times A_{K}^{1}[0, a]$ if we replace $a$ by a smaller value. Define the matrix $N=$ 
$\left(N_{j l}\right) \in \operatorname{Mat}_{n}\left(\mathcal{O}\left(X \times A_{K}^{1}[0, a]\right)\right)$ by $\partial\left(\mathbf{e}_{l}\right)=\sum_{j=1}^{n} N_{j l} \mathbf{e}_{j}$ and write $N=\sum_{i=0}^{\infty} N_{i} t^{i}$, $N_{i} \in \operatorname{Mat}_{n}(\mathcal{O}(X))$.

First we prove the lemma in the case $s=0$. The key claim for the proof is the following:

claim.

(1) There exists a unique matrix $M \in \operatorname{Mat}_{n}(\mathcal{O}(X)[[t]])$ congruent to identity modulo $t$ satisfying $N M+\partial M=M N_{0}$.

(2) The matrix $M$ in (1) converges on $X \times A_{K}^{1}[0, b]$ for some $b \in(0, a] \cap \Gamma^{*}$.

Let us prove the assertion (1) of the claim. If we write $M=\sum_{i=0}^{\infty} M_{i} t^{i}$ with $M_{0}$ identity, we should have

$$
i M_{i}+N_{0} M_{i}-M_{i} N_{0}=-\sum_{j=0}^{i-1} N_{i-j} M_{j}
$$

for $i>0$. Let $g: \operatorname{Mat}_{n}(\mathcal{O}(X)) \longrightarrow \operatorname{Mat}_{n}(\mathcal{O}(X))$ be the $\mathcal{O}(X)$-linear map defined by $g(Y)=N_{0} Y-Y N_{0}$. If we know that $g+i \cdot$ id is invertible for $i>0$, we can uniquely determine $M_{i}$ 's $(i \geq 0)$ inductively by the equation (1.1). So it suffice to prove that $g+i \cdot$ id $(i>0)$ is invertible. To prove this, we may enlarge $K$.

Since $N_{0}$ is the residue of $E$ along $\{t=0\}$, there exists a polynomial $P(x) \in K[x]$ of the form $P(x)=\prod_{i=1}^{m}\left(x-\xi_{i}\right)^{n_{i}}$ with $\xi_{i} \in \Sigma_{r+1}$ such that $P\left(N_{0}\right)=0$ holds. By enlarging $K$, we may assume that $\xi_{i}$ 's belong to $K$. Then we have a decomposition

$$
\mathcal{O}(X)^{n}=\left.E\right|_{X \times\{0\}}=\bigoplus_{k=1}^{m} E_{k}
$$

such that $N_{0}-\xi_{k}$. id acts on $E_{k}$ nilpotently. According to this decomposition, we have the decomposition

$$
\operatorname{Mat}_{n}(\mathcal{O}(X))=\operatorname{End}_{\mathcal{O}(X)}\left(\left.E\right|_{X \times\{0\}}\right)=\bigoplus_{k, l=1}^{m} \operatorname{Hom}_{\mathcal{O}(X)}\left(E_{k}, E_{l}\right),
$$

and on each $\operatorname{Hom}_{\mathcal{O}(X)}\left(E_{k}, E_{l}\right), g$ is defined by $g(Y)=\left(N_{0}-\xi_{l} \cdot\right.$ id $)(Y)-Y\left(N_{0}-\xi_{k}\right.$. id $)+\left(\xi_{l}-\xi_{k}\right) Y$. Hence $g-\left(\xi_{l}-\xi_{k}\right) \cdot \mathrm{id}=(g+i \cdot \mathrm{id})-\left(\xi_{l}-\xi_{k}-i\right)$ acts nilpotently on $\operatorname{Hom}_{\mathcal{O}(X)}\left(E_{k}, E_{l}\right)$. Since $\Sigma_{r+1}$ is (NID), $\xi_{l}-\xi_{k}-i$ is non-zero for any $k, l$. It implies that $g+i \cdot$ id is invertible. So we have proved the assertion (1).

Let us prove the assertion (2) of the claim. Since $N$ is in $\operatorname{Mat}_{n}\left(\mathcal{O}\left(X \times A_{K}^{1}[0, a]\right)\right)$, there exists a constant $C>1$ satisfying $\left|N_{i}\right| \leq C a^{-i}$. (Here || denotes any fixed Banach norm on $X$ andd || for a matrix is defined as the maximum of the norms of its entries.) Let us define $\mathcal{O}(X)$-linear maps $h_{1, i}, h_{2}: \operatorname{Mat}_{n}(\mathcal{O}(X)) \longrightarrow \operatorname{Mat}_{n}(\mathcal{O}(X))$ $(i>0)$ by the composite maps

$$
\operatorname{Mat}_{n}(\mathcal{O}(X))=\bigoplus_{k, l=1}^{m} \operatorname{Hom}_{\mathcal{O}(X)}\left(E_{k}, E_{l}\right) \longrightarrow \bigoplus_{k, l=1}^{m} \operatorname{Hom}_{\mathcal{O}(X)}\left(E_{k}, E_{l}\right)=\operatorname{Mat}_{n}(\mathcal{O}(X))
$$


where the arrow in the middle is defined by $\left(Y_{k l}\right)_{k, l} \mapsto\left(\left(\xi_{l}-\xi_{k}+i\right) Y_{k l}\right)_{k, l}$ in the case of $h_{1, i}$ and $\left(Y_{k l}\right)_{k, l} \mapsto\left(\left(g-\left(\xi_{l}-\xi_{k}\right) \text { id }\right)\left(Y_{k l}\right)\right)_{k, l}$ in the case of $h_{2}$. Then we have $g+i \cdot \operatorname{id}=h_{1, i}+h_{2}$ and $h_{1, i}$ (resp. $\left.h_{2}\right)$ is invertible (resp. nilpotent). Let us take a natural number $e$ satisfying $h_{2}^{e}=0$. Then the inverse of the map $g+i \cdot$ id is given by $(g+i \cdot \mathrm{id})^{-1}=\sum_{j=0}^{e-1}(-1)^{j}\left(h_{1, i}^{-1}\right)^{j+1} h_{2}^{j}$.

Let us replace the constant $C$ by a larger value so that it satisfies the inequalities $\left|h_{1, i}^{-1}\right| \leq C \max _{k, l}\left|\left(\xi_{l}-\xi_{k}+i\right)^{-1}\right|,\left|h_{2}\right| \leq C$ and let us put

$$
A_{i}:=\prod_{j=1}^{i} \max \left(\max _{k, l}\left|\left(\xi_{l}-\xi_{k}+j\right)^{-1}\right|, 1\right) .
$$

With these notation, we prove the inequality $\left|M_{i}\right| \leq A_{i}^{e} C^{2 e i} a^{-i}$ by induction on $i$. It is obviously true for $i=0$. If the inequality is true for $i-1$, we have

$$
\begin{aligned}
\left|(g+i \cdot \mathrm{id})\left(M_{i}\right)\right| & =\left|\sum_{j=0}^{i-1} N_{i-j} M_{j}\right| \\
& \leq \max _{0 \leq j \leq i-1}\left(C a^{-i+j} \cdot A_{j}^{e} C^{2 e j} a^{-j}\right) \leq A_{i-1}^{e} C^{2 e(i-1)+1} a^{-i} .
\end{aligned}
$$

Hence we have

$$
\begin{aligned}
\left|M_{i}\right| & =\left|\sum_{j=0}^{e-1}(-1)^{j}\left(h_{1, i}^{-1}\right)^{j+1} h_{2}^{j}\left((g+i \cdot \mathrm{id})\left(M_{i}\right)\right)\right| \\
& \leq \max _{0 \leq j \leq e-1}\left(C^{j+1}\left(\max _{k, l}\left|\left(\xi_{l}-\xi_{k}+i\right)^{-1}\right|\right)^{j+1} C^{j}\right) \cdot A_{i-1}^{e} C^{2 e(i-1)+1} a^{-i} \\
& \leq A_{i}^{e} C^{2 e i} a^{-i}
\end{aligned}
$$

and so the desired inequality is proved.

By [9, VI, Lemma 1.2], we have an equality of the series

$$
\sum_{s=0}^{\infty} \frac{X^{s}}{\prod_{i=1}^{s}(i-\alpha)}=\alpha e^{X} \sum_{s=0}^{\infty} \frac{(-X)^{s}}{s !} \cdot \frac{1}{\alpha-s}
$$

for $\alpha \in \bar{K}-\mathbb{N}$. From this, we easily see that the radius of convergence of the left hand side is positive if type $(\alpha)$ is positive. Putting $\alpha=\xi_{k}-\xi_{l}$ (which is in $\bar{K}-\mathbb{N}$ with positive type since $\Sigma_{r+1}$ is (NID) and (PTD)), we see that, for some $\rho>0$, the inequality $A_{i}<\rho^{-i}$ holds for all $i>0$. Then, if we take $b \in(0, a] \cap \Gamma^{*}$ to be a number satisfying $0<b<\rho^{e} C^{-2 e} a$, we have $\left|M_{i}\right|<b^{-i}$ for all $i>0$. Hence $M$ converges on $X \times A_{K}^{1}[0, b]$ and so the assertion (2) of the claim is proved.

By the claim, the matrix $M=\left(M_{j l}\right)$ is an invertible matrix in $\operatorname{Mat}_{n}(\mathcal{O}(X \times$ $\left.\left.A_{K}^{1}[0, b]\right)\right)$. Define the vectors $\mathbf{v}_{1}, \ldots, \mathbf{v}_{n} \in \Gamma\left(X \times A_{K}^{1}[0, b], E\right)$ by $\mathbf{v}_{l}=\sum_{j} M_{j l} \mathbf{e}_{j}$. Then $\mathbf{v}_{1}, \ldots, \mathbf{v}_{n}$ forms a basis of $E$ on $X \times A_{K}^{1}[0, b]$ and the operator $\partial$ acts on this basis via the matrix $N_{0}$. Also, by the formal power series computation, we see that 
the $\mathcal{O}_{X^{-}}$-span of this basis (which we denote by $F$ ) is characterized as the subspace of $E$ on which $P(\partial)$ acts as zero. Hence the restriction of $(E, \nabla)$ to $F$ naturally defines a $\log -\nabla$-module on $X$ whose exponents along $\left\{x_{i}=0\right\}$ are in $\Sigma_{i}(1 \leq i \leq r)$, endowed with the operator $\partial=N_{0}$. That is, $F$ defines an object in $\operatorname{LNM}_{X \times A_{K}^{1}[0,0], \Sigma}$ and it is easy to see that $E=\mathcal{U}_{[0, b]}(F)$ holds. Hence the proof of the lemma is finished in the case $s=0$.

Next let us treat the general case. Let us rewrite $(E, \nabla)$ as $\left(\left(E, \nabla^{\prime}\right),\left\{\partial_{i}\right\}_{i=1}^{s}\right)$, where $\left(E, \nabla^{\prime}\right) \in \operatorname{LNM}_{X \times A_{K}^{1}[0, a], \prod_{i=1}^{r+1} \Sigma_{i}}$ and $\partial_{i}(1 \leq i \leq s)$ are commuting endomorphisms on $\left(E, \nabla^{\prime}\right)$ with eigenvalues in $\Sigma_{r+i+1}$. Then we can apply the argument up to the previous paragraph to $\left(E, \nabla^{\prime}\right)$ : So let us take $\partial, b, N_{0}$, the basis $\mathbf{v}_{1}, \ldots, \mathbf{v}_{n} \in \Gamma\left(X \times A_{K}^{1}[0, b], E\right)$ and $F$ as above, and let $L_{i}=\sum_{j=0}^{\infty} L_{i j} t^{j}(1 \leq i \leq s)$ be the matrix expression of $\partial_{i}$ with respect to the basis $\mathbf{v}_{1}, \ldots, \mathbf{v}_{n}$. Since $\partial_{i}$ 's are compatible with $\partial$, we have equalities $N_{0} L_{i}+\partial L_{i}=L_{i} N_{0}(1 \leq i \leq s)$, that is, the equalities

$$
j L_{i j}+N_{0} L_{i j}-L_{i j} N_{0}=O \quad(1 \leq i \leq s, j \geq 0) .
$$

As we see above, the map $Y \mapsto j Y+N_{0} Y-Y N_{0}$ is invertible for any positive integer $j$. Hence $L_{i j}=O$ for $j>0$, that is, $\partial_{i}$ 's come from endomorphisms of $F$. So $\left(\left(F,\left.\nabla^{\prime}\right|_{F}\right),\left\{\left.\partial_{i}\right|_{F}\right\}_{i=1}^{s}\right)$ naturally defines an object in $\operatorname{LNM}_{X \times A_{K}^{1}[0,0] \times A_{K}^{s}[0,0], \Sigma}$ which is sent to $\left(\left(E, \nabla^{\prime}\right),\left\{\partial_{i}\right\}_{i=1}^{s}\right)=(E, \nabla)$ by $\mathcal{U}_{[0, b]}^{\prime}$. So the proof in general case is also finished.

Using Lemma 1.9, we obtain the following (cf. [10, 3.2.12]):

Lemma 1.10. Let $X$ be a smooth rigid space endowed with $x_{1}, \ldots, x_{r} \in \Gamma\left(X, \mathcal{O}_{X}\right)$ whose zero loci are smooth and meet transversally. Let $a \in(0, \infty) \cap \Gamma^{*}$, let $\Sigma=$ $\prod_{i=1}^{r+n+m} \Sigma_{i}$ be a subset of $\bar{K}^{r+n+m}$ such that $\prod_{i=r+1}^{r+n+m} \Sigma_{i}$ is (NID) and (PTD) and let $(E, \nabla)$ be an object in $\mathrm{LNM}_{X \times A_{K}^{n}[0, a] \times A_{K}^{m}[0,0], \Sigma}$ such that $\left.E\right|_{X \times\{0\}}$ is free. Then, there exists $b \in(0, a] \cap \Gamma^{*}$ such that the restriction of $(E, \nabla)$ to $X \times A_{K}^{n}[0, b] \times A_{K}^{m}[0,0]$ is in the essential image of $\mathcal{U}_{[0, b]}^{\prime}: \operatorname{LNM}_{X \times A_{K}^{n+m}[0,0], \Sigma} \longrightarrow \operatorname{LNM}_{X \times A_{K}^{n}[0, b] \times A_{K}^{m}[0,0], \Sigma}$, where $\mathcal{U}_{[0, b]}^{\prime}$ is the functor naturally induced by $\mathcal{U}_{[0, b]}: \operatorname{LNM}_{X \times A_{K}^{n}[0,0], \prod_{i=1}^{r+n} \Sigma_{i}} \longrightarrow$ $\mathrm{LNM}_{X \times A_{K}^{n}[0, b], \prod_{i=1}^{r+n} \Sigma_{i}}$.

Proof. We prove the lemma by induction on $n$. Let $i: X \times A_{K}^{n-1}[0, a] \times A_{K}^{m+1}[0,0] \hookrightarrow$ $X \times A_{K}^{n}[0, a] \times A_{K}^{m}[0,0]$ be the zero locus of the $n$-th coordinate function of $A_{K}^{n}[0, a]$. Then, by induction hypothesis, $i^{*}(E, \nabla)$ is in the essential image of

$$
\mathcal{U}_{[0, b]}^{\prime}: \operatorname{LNM}_{X \times A_{K}^{n+m}[0,0], \Sigma} \longrightarrow \operatorname{LNM}_{X \times A_{K}^{n-1}[0, b] \times A_{K}^{m+1}[0,0], \Sigma}
$$

for some $b$. Then, if we write $i^{*}(E, \nabla)=\mathcal{U}_{[0, b]}^{\prime}\left(\left(E^{\prime}, \nabla^{\prime}\right)\right)$, we have the isomorphism

$$
\left.E\right|_{X \times\{0\}}=\left.\left(i^{*} E\right)\right|_{X \times\{0\}}=\left.\left(\pi^{*} E^{\prime}\right)\right|_{X \times\{0\}}=E^{\prime},
$$

where $\pi: X \times A_{K}^{n-1}[0, b] \longrightarrow X$ denotes the projection. Hence $E^{\prime}$ is free and so $i^{*} E=\pi^{*} E^{\prime}$ is also free. Hence we may apply Lemma [1.9 and we see that the 
restriction of $(E, \nabla)$ to $X \times A_{K}^{n}[0, b] \times A_{K}^{m}[0,0]$ is in the essential image of

$$
\mathcal{U}_{[0, b]}^{\prime}: \operatorname{LNM}_{X \times A_{K}^{n-1}[0, b] \times A_{K}^{m+1}[0,0], \Sigma} \longrightarrow \operatorname{LNM}_{X \times A_{K}^{n}[0, b] \times A_{K}^{m}[0,0], \Sigma}
$$

for some smaller $b$. Then, by using the induction hypothesis again, we see that the restriction of $(E, \nabla)$ to $X \times A_{K}^{n}[0, b] \times A_{K}^{m}[0,0]$ is in the essential image of

$$
\mathcal{U}_{[0, b]}^{\prime}: \operatorname{LNM}_{X \times A_{K}^{n+m}[0,0], \Sigma} \longrightarrow \operatorname{LNM}_{X \times A_{K}^{n}[0, b] \times A_{K}^{m}[0,0], \Sigma}
$$

for some even smaller $b$, as desired.

Using Lemma 1.10, we can prove the following proposition, which is the analogue of [10, 3.2.14]:

Proposition 1.11. Let $X$ be a smooth rigid space endowed with $x_{1}, \ldots, x_{r} \in \Gamma\left(X, \mathcal{O}_{X}\right)$ whose zero loci are smooth and meet transversally. Let $\Sigma=\prod_{i=1}^{r} \Sigma_{i}$ be a subset of $\bar{K}^{r}$ which is (NID) and (PTD). Then, for a morphism $f:\left(E, \nabla_{E}\right) \longrightarrow\left(F, \nabla_{F}\right)$ in $\operatorname{LNM}_{X, \Sigma}, \operatorname{Ker}(f)$ and $\operatorname{Coker}(f)$ endowed with canonical connections are also in the category $\mathrm{LNM}_{X, \Sigma}$. In particular, $\mathrm{LNM}_{X, \Sigma}$ is an abelian category.

Proof. It suffices to prove that $\operatorname{Ker}(f)$ and $\operatorname{Coker}(f)$ are locally free. So we may replace $K$ by a finite extension and it suffices to check the local freeness on a neighborhood of each point of $X$ (see [10, 3.2.13]). Hence, by [10, 2.3.3] (see also the proof of [10, 3.2.14], [12, 1.18]), we may assume $X=A_{K}^{n}[0, a]$ for some $n \geq r$ and $a \in(0, \infty) \cap \Gamma^{*}$ such that $x_{i}(1 \leq i \leq r)$ are a part of the coordinate of $A_{K}^{n}[0, a]$. Then, by replacing $\Sigma$ by $\Sigma \times\{0\}^{n-r}$, we may assume that the exponents of $E$ and $F$ are contained in $\Sigma$ and by Lemma 1.10 (with $X=\operatorname{Spm} K$ in the notation there), we may assume that $E$ and $F$ are in the image of $\mathcal{U}_{[0, a]}: \operatorname{LNM}_{A_{K}^{n}[0,0], \Sigma} \longrightarrow \operatorname{LNM}_{A_{K}^{n}[0, a], \Sigma}$. Moreover, by enlarging $K$, we may assume that $\Sigma \subseteq K^{n}$.

Let $\mathbf{e}_{1}, \ldots, \mathbf{e}_{l}$ (resp. $\left.\mathbf{f}_{1}, \ldots, \mathbf{f}_{m}\right)$ be a basis of $E\left(\right.$ resp. $F$ ) on which $\partial_{i}:=t_{i} \frac{\partial}{\partial t_{i}}$ acts via matrices over $K$. We can assume moreover that there exists an element $\xi:=\left(\xi_{1}, \ldots, \xi_{r}\right) \in \Sigma$ such that $\partial_{i}\left(\mathbf{e}_{1}\right)=\xi_{i} \mathbf{e}_{1}$. Let us consider the following claim:

claim. $f\left(\mathbf{e}_{1}\right)$ is contained in the $K$-span of $\mathbf{f}_{1}, \ldots, \mathbf{f}_{m}$.

If this is true, we can prove the local freeness of $\operatorname{Ker}(f)$ and $\operatorname{Coker}(f)$ in the same way as [10, 3.2.14], by induction on the rank of $E \oplus F$. Hence it suffices to prove the above claim.

Let us put $f\left(\mathbf{e}_{1}\right)=\sum_{i=1}^{m} a_{i}(t) \mathbf{f}_{i}$. Then, to prove the claim, it suffices to prove the following statement $(*)_{j}$ for each $1 \leq j \leq n$ :

$(*)_{j}$ : For $1 \leq i \leq m, a_{i}(t)$ are constant with respect to the variable $t_{j}$.

By symmetry with respect to the variables, it suffices to prove $(*)_{1}$. To prove 
this, we may change the basis $\mathbf{f}_{1}, \ldots, \mathbf{f}_{m}$ by another basis of the $K$-span of $\mathbf{f}_{1}, \ldots, \mathbf{f}_{m}$. So we may assume that there exist elements $\eta_{1}, \ldots, \eta_{k} \in \Sigma_{1}$ and the sequence $0=m_{0}<m_{1}<m_{2}<\cdots<m_{k}=m$ satisfying

$$
\partial_{1}\left(\mathbf{f}_{m_{i}}\right)=\eta_{i} \mathbf{f}_{m_{i}}(1 \leq i \leq k), \partial_{1}\left(\mathbf{f}_{j}\right)=\eta_{i} \mathbf{f}_{j}+\mathbf{f}_{j+1}\left(m_{i}<j<m_{i+1}\right) .
$$

Let us define $\bar{\nabla}_{E}, \bar{\nabla}_{F}$ as the composite map

$$
\begin{aligned}
& E \stackrel{\bar{\nabla}_{E}}{\longrightarrow} E \otimes \omega_{A_{K}^{n}[0, a] / K}^{1} \longrightarrow E \otimes \omega_{A_{K}^{n}[0, a] / A_{K}^{n-1}[0, a]}^{1}=E \overline{\operatorname{dlog} t_{1}}, \\
& F \stackrel{\bar{\nabla}_{E}}{\longrightarrow} F \otimes \omega_{A_{K}^{n}[0, a] / K}^{1} \longrightarrow F \otimes \omega_{A_{K}^{n}[0, a] / A_{K}^{n-1}[0, a]}^{1}=F \overline{\operatorname{dlog} t_{1}},
\end{aligned}
$$

respectively. Then we have

$$
f\left(\bar{\nabla}_{E}\left(\mathbf{e}_{1}\right)\right)=f\left(\xi_{1} \mathbf{e}_{1} \overline{\operatorname{dlog} t_{1}}\right)=\left(\sum_{i=1}^{m} \xi_{1} a_{i}(t) \mathbf{f}_{i}\right) \overline{\operatorname{dlog} t_{1}} .
$$

On the other hand, we have

$$
\begin{gathered}
\bar{\nabla}_{F}\left(f\left(\mathbf{e}_{1}\right)\right)=\bar{\nabla}_{F}\left(\sum_{i=1}^{m} a_{i}(t) \mathbf{f}_{i}\right)=\left(\sum_{i=1}^{m} \frac{\partial a_{i}}{\partial t_{1}}(t) t_{1} \mathbf{f}_{i}+\sum_{i=1}^{m} a_{i}(t) \partial_{1}\left(\mathbf{f}_{i}\right)\right) \\
=\sum_{i=0}^{k-1}\left\{\left(\frac{\partial a_{m_{i}+1}}{\partial t_{1}}(t) t_{1}+\eta_{i+1} a_{m_{i}+1}(t)\right) \mathbf{f}_{m_{i}+1}\right. \\
\left.+\sum_{j=m_{i}+2}^{m_{i+1}}\left(\frac{\partial a_{j}}{\partial t_{1}}(t) t_{1}+\eta_{i+1} a_{j}(t)+a_{j-1}(t)\right) \mathbf{f}_{j}\right\} \overline{\operatorname{dog} t_{1}}
\end{gathered}
$$

By comparing (1.2) and (1.3), we obtain the equalities

$$
\begin{aligned}
& \left(\xi_{1}-\eta_{i+1}\right) a_{m_{i}+1}(t)=\frac{\partial a_{m_{i}+1}}{\partial t_{1}}(t) t_{1} \quad(0 \leq i \leq k-1) \\
& \left(\xi_{1}-\eta_{i+1}\right) a_{j}(t)=\frac{\partial a_{j}}{\partial t_{1}}(t) t_{1}+a_{j-1}(t), \quad\left(0 \leq i \leq k-1, m_{i}+2 \leq j \leq m_{i+1}\right) .
\end{aligned}
$$

Since $\xi_{1}-\eta_{i+1}$ is not a non-zero integer, we see from (1.4) that $a_{m_{i}+1}(t)(0 \leq i \leq k-1)$ are constant with respect to $t_{1}$. From this, (1.5) and the induction on $j$, we see that $a_{j}(t)\left(0 \leq i \leq k-1, m_{i}+2 \leq j \leq m_{i+1}\right)$ are also constant with respect to $t_{1}$. So we have proved the assertion $(*)_{1}$ and so we are done.

Corollary 1.12. Let $X$ be a smooth rigid space endowed with $x_{1}, \ldots, x_{r} \in \Gamma\left(X, \mathcal{O}_{X}\right)$ whose zero loci are smooth and meet transversally. Let $\Sigma=\prod_{i=1}^{r+n} \Sigma_{i}$ be a subset of $\bar{K}^{r+n}$ such that $\Sigma=\prod_{i=1}^{r} \Sigma_{i}$ is (NID) and (PTD). Then $\operatorname{LNM}_{X \times A_{K}^{n}[0,0], \Sigma}$ is an abelian category.

Proof. Immediate from Proposition 1.11, 
We have the following remark generalizing Remark 1.6, which is the analogue of [10, 3.2.16].

Remark 1.13. Let $X$ be a connected smooth rigid space endowed with $x_{1}, \ldots, x_{r} \in$ $\Gamma\left(X, \mathcal{O}_{X}\right)$ whose zero loci are smooth and meet transversally. Let $\Sigma:=\prod_{i=1}^{r+n} \Sigma_{i}$ be a subset of $\bar{K}^{r+n}$ such that $\Sigma_{i}(1 \leq i \leq r)$ are (NID) and (PTD) and let $I$ be an aligned subinterval of $[0, \infty)$.

(1) In the case $I=[0,0]$, a $\log$ - $\nabla$-module on $X \times A_{K}^{n}(I)$ is potentially $\Sigma$-unipotent if and only if it is in the category $\operatorname{LNM}_{X \times A_{K}^{n}[0,0], \Sigma}$.

We give a proof of this assertion by induction on the rank. (It suffices to prove that an object in $\operatorname{LNM}_{X \times A_{K}^{n}[0,0], \Sigma}$ is potentially $\Sigma$-unipotent.) Let us take an object in $\operatorname{LNM}_{X \times A_{K}^{n}[0,0], \Sigma}$, regarded as an object $(E, \nabla)$ in $\mathrm{LNM}_{X, \prod_{i=1}^{r} \Sigma_{i}}$ endowed with commuting endomorphisms $\partial_{i}(1 \leq i \leq n)$ such that all the eigenvalues of $\partial_{i}$ are in $\Sigma_{i}$ for any $1 \leq i \leq n$. We may enlarge $K$ in order that all the eigenvalues are in $K$. Since $\partial_{i}$ are commuting endomorphisms of $(E, \nabla), E_{1}:=\bigcap_{i=1}^{n} \operatorname{Ker}\left(\partial_{i}-\xi_{i}\right)$ is an subobject of $(E, \nabla)$ in $\operatorname{LNM}_{X, \prod_{i=1}^{r} \Sigma_{i}}$ and it is non-zero for some $\left(\xi_{1}, \ldots, \xi_{n}\right) \in \prod_{i=1}^{n} \Sigma_{r+i}$. It is easy to see that $E_{1}$ is $\Sigma$-constant, and the quotient $E / E_{1}$ is potentially $\Sigma$-unipotent by induction hypothesis. Hence $E$ is potentially $\Sigma$-unipotent, as desired.

The same argument shows that, in the case $I=[0,0]$ and $\prod_{i=r+1}^{r+n} \Sigma_{i} \subseteq K^{n}$, a $\log$ - $\nabla$-module on $X \times A_{K}^{n}(I)$ is $\Sigma$-unipotent if and only if it is in the category $\operatorname{LNM}_{X \times A_{K}^{n}[0,0], \Sigma}$. In particular, $\Sigma$-unipotence is equivalant to potential $\Sigma$-unipotence in this case.

(2) From (1), we easily see that, under the assumption here (with $I$ arbitrary), an object in the essential image of the functor $\mathcal{U}_{I}: \operatorname{ULNM}_{X \times A_{K}^{n}[0,0], \Sigma}^{\prime}=$ $\operatorname{LNM}_{X \times A_{K}^{n}[0,0], \Sigma} \longrightarrow \operatorname{LNM}_{X \times A_{K}^{n}(I), \Sigma}$ is potentially $\Sigma$-unipotent, and it is $\Sigma$ unipotent if $\prod_{i=r+1}^{r+n} \Sigma_{i} \subseteq K^{n}$.

Next we prove an important property of the functor $\mathcal{U}_{I}$, which is an analogue of [10, 3.3.2]:

Proposition 1.14. Let $X$ be a smooth rigid space endowed with $x_{1}, \ldots, x_{r} \in \Gamma\left(X, \mathcal{O}_{X}\right)$ whose zero loci are smooth and meet transversally and let I be a quasi-open subinterval of positive length in $[0, \infty)$. Let $\Sigma=\prod_{i=1}^{r+n} \Sigma_{i}$ be a subset of $\bar{K}^{r+n}$ which is (NID) and (NLD). Then, for any objects $E, E^{\prime}$ in $\mathrm{ULNM}_{X \times A_{K}^{n}[0,0], \Sigma}^{\prime}\left(=\operatorname{LNM}_{X \times A_{K}^{n}[0,0], \Sigma}\right.$ by Remark 1.13), the canonical morphism

$$
\operatorname{Ext}^{i}\left(E, E^{\prime}\right) \longrightarrow \operatorname{Ext}^{i}\left(\mathcal{U}_{I}(E), \mathcal{U}_{I}\left(E^{\prime}\right)\right)
$$

is an isomorphism for $i=0,1$. (Here $\mathrm{Ext}^{i}$ denotes the extension group in the category $\operatorname{LNM}_{X \times A_{K}^{n}[0,0], \Sigma}$ and $\operatorname{LNM}_{X \times A_{K}^{n}(I), \Sigma}$. 
Proof. Let us put $F=E^{\vee} \otimes E^{\prime}$ (as $\log -\nabla$-module on $X \times A_{K}^{n}[0,0]$ with respect to $\left.x_{i}, \ldots, x_{r}, t_{1}, \ldots, t_{n}\right)$. Then, by [10, 3.3.1], we have

$$
\begin{aligned}
\operatorname{Ext}^{i}\left(E, E^{\prime}\right) & \cong H^{i}\left(X, F \otimes \omega_{X \times A_{K}^{n}[0,0] / K}^{\bullet}\right), \\
\operatorname{Ext}^{i}\left(\mathcal{U}_{I}(E), \mathcal{U}_{I}\left(E^{\prime}\right)\right) & \cong H^{i}\left(X, \mathcal{U}_{I}(F) \otimes \omega_{X \times A_{K}^{n}(I) / K}^{\bullet} .\right.
\end{aligned}
$$

Hence it suffices to prove that the map

$$
H^{i}\left(X, F \otimes \omega_{X \times A_{K}^{n}[0,0] / K}^{\bullet}\right) \longrightarrow H^{i}\left(X, \mathcal{U}_{I}(F) \otimes \omega_{X \times A_{K}^{n}(I) / K}^{\bullet}\right)
$$

is an isomorphism for $i \geq 0$. To prove this, we can enlarge $K$. So, by five lemma and the exactness of $\mathcal{U}_{I}$, we can reduce to the case where $E, E^{\prime}$ are $\Sigma$-constant. Also, we can reduce to the case where $X$ is affinoid (by considering the Cech spectral sequence), and we can assume that $F$ is a free $\mathcal{O}_{X}$-module. Hence $F$ has the form $F_{0} \otimes \pi^{*}\left(M_{-\xi} \otimes M_{\xi^{\prime}}\right)$ for some $\xi, \xi^{\prime} \in \prod_{i=1}^{n} \Sigma_{r+i}$ and for some log- $\nabla$-module $F_{0}$ on $X$ with respect to $x_{1}, \ldots, x_{r}$ with $F_{0}$ free as $\mathcal{O}_{X}$-module. (Here $\pi$ denotes the projection $X \times A_{K}^{n}[0,0] \longrightarrow A_{K}^{n}[0,0]$.) In particular, if we regard $F$ as a log- $\nabla$-module on $X \times A_{K}^{n}[0,0]$ with respect to $t_{1}, \ldots, t_{n}$ relative to $X$, it is a finite direct sum of $\pi^{*}\left(M_{-\xi} \otimes M_{\xi^{\prime}}\right)$.

Now let us consider the Katz-Oda type spectral sequence for the diagram $X \times$ $A_{K}^{n}[0,0] \longrightarrow X \longrightarrow \operatorname{Spm} K$ and the diagram $X \times A_{K}^{n}(I) \longrightarrow X \longrightarrow \operatorname{Spm} K$. From the first diagram, we obtain the spectral sequence

$E_{2}^{p, q}=H^{p}\left(\Gamma\left(X, \omega_{X / K}^{\bullet}\right) \otimes H^{q}\left(X, F \otimes \omega_{X \times A_{K}^{n}[0,0] / X}^{\bullet}\right)\right) \Longrightarrow H^{p+q}\left(X, F \otimes \omega_{X \times A_{K}^{n}[0,0] / K}^{\bullet}\right)$

and from the second diagram, we obtain the spectral sequence

$$
\begin{aligned}
E_{2}^{p, q} & =H^{p}\left(\Gamma\left(X, \omega_{X / K}^{\bullet}\right) \otimes H^{q}\left(X \times A_{K}^{n}(I), \mathcal{U}_{I}(F) \otimes \omega_{X \times A_{K}^{n}(I) / X}^{\bullet}\right)\right) \\
& \Longrightarrow H^{p+q}\left(X \times A_{K}^{n}(I), \mathcal{U}_{I}(F) \otimes \omega_{X \times A_{K}^{n}(I) / K}^{\bullet} .\right.
\end{aligned}
$$

Hence it suffices to prove that the map

$$
H^{q}\left(X, F \otimes \omega_{X \times A_{K}^{n}[0,0] / X}^{\bullet} \longrightarrow H^{q}\left(X \times A_{K}^{n}(I), \mathcal{U}_{I}(F) \otimes \omega_{X \times A_{K}^{n}(I) / X}^{\bullet}\right)\right.
$$

is an isomorphism. Since the map (1.6) depends only on the structure of $F$ as a log$\nabla$-module on $X \times A_{K}^{n}[0,0]$ with respect to $t_{1}, \ldots, t_{n}$ relative to $X$, we may suppose that $F$ has the form $\pi^{*}\left(M_{-\xi} \otimes M_{\xi^{\prime}}\right)=\pi^{*}\left(M_{\alpha}\right)$, where we put $\alpha_{i}:=\xi_{i}^{\prime}-\xi_{i}, \alpha:=\left(\alpha_{1}, \ldots, \alpha_{n}\right)$. In this case, the map (1.6) is the map between cohmologies induced by the following map of complexes

$$
g: \Gamma\left(X, \omega_{X \times A_{K}^{n}[0,0] / X}^{\bullet} \longrightarrow \Gamma\left(X \times A_{K}^{n}(I), \omega_{X \times A_{K}^{n}(I) / X}^{\bullet},\right.\right.
$$

where the complexes $\omega_{X \times A_{K}^{n}[0,0] / X}^{\bullet}, \omega_{X \times A_{K}^{n}(I) / X}^{\bullet}$ are the relative de Rham complex associated to the map $d+\sum_{i=1}^{n} \alpha_{i} \mathrm{~d} \log t_{i}$. Let

$$
h: \Gamma\left(X \times A_{K}^{n}(I), \omega_{X \times A_{K}^{n}(I) / X}^{\bullet} \longrightarrow \Gamma\left(X, \omega_{X \times A_{K}^{n}[0,0] / X}^{\bullet}\right)\right.
$$


be the map induced by the map $\mathcal{O}\left(X \times A_{K}^{n}(I)\right) \longrightarrow \mathcal{O}(X)$ of 'taking the constant coefficient'. Then the composite $h \circ g$ is the identity. Hence it suffices to prove that the composite map $g \circ h$ is homotopic to the identity. We construct the homotopy $\varphi$ in a similar way to [10,3.3.2]. For a monomial of the form

$$
t_{1}^{i_{1}} \cdots t_{n}^{i_{n}} \operatorname{dlog} t_{j_{1}} \wedge \cdots \wedge \operatorname{dlog} t_{j_{k}}, \quad\left(i_{1}, \ldots, i_{n} \in \mathbb{Z}, 1 \leq j_{1}<\cdots j_{k} \leq n\right),
$$

let $l:=l\left(i_{1}, \ldots, i_{n}\right)$ be the least integer with $i_{l} \neq 0$ and send it by $\varphi$ to 0 if $l \notin$ $\left\{j_{1}, \ldots, j_{k}\right\}$ and to

$$
\frac{1}{i_{l}+\alpha_{l}} t_{1}^{i_{1}} \cdots t_{n}^{i_{n}} \operatorname{dlog} t_{j_{1}} \wedge \cdots \operatorname{dlog} t_{j_{s-1}} \wedge \operatorname{dlog} t_{j_{s+1}} \wedge \cdots \operatorname{dlog} t_{j_{k}}
$$

when $l=j_{s}$. (It is well-defined because $\alpha_{l}$ is not a non-zero integer.) We claim that we can extend $\varphi$ in natural way to define a map

$$
\varphi: \Gamma\left(X \times A_{K}^{n}(I), \omega_{X \times A_{K}^{n}(I) / X}^{k}\right) \longrightarrow \Gamma\left(X \times A_{K}^{n}(I), \omega_{X \times A_{K}^{n}(I) / X}^{k-1}\right) .
$$

To prove this, it suffices to prove that, for a formal power series $\sum_{\mathbf{i}} a_{\mathbf{i}} t_{1}^{i_{1}} \cdots t_{n}^{i_{n}}$ (where $\mathbf{i}=\left(i_{1}, \ldots, i_{n}\right)$ and $a_{\mathbf{i}} \in \Gamma\left(X, \mathcal{O}_{X}\right)$ ) convergent on $X \times A_{K}^{n}(I)$, so is the series $\sum_{\mathbf{i}} \frac{a_{\mathbf{i}}}{i_{l(\mathbf{i})}+\alpha_{l(\mathbf{i})}} t_{1}^{i_{1}} \cdots t_{n}^{i_{n}}$. We can prove this claim easily by using the fact that $\alpha_{1}, \ldots, \alpha_{n}$ are $p$-adically non-Liouville (which follows from the assumption that $\Sigma$ is (NLD)). Then we can easily check by formal power series computation that the map $\varphi$ gives a homotopy between $g \circ h$ and the identity. So we are done.

Corollary 1.15 (cf. [10, 3.3.4]). Let $X, x_{1}, \ldots, x_{r}, I, \Sigma$ be as in Proposition 1.14. Then $\mathcal{U}_{I}$ induces equivalences of categories

$$
\mathrm{ULNM}_{X \times A_{K}^{n}[0,0], \Sigma} \stackrel{=}{\longrightarrow} \operatorname{ULNM}_{X \times A_{K}^{n}(I), \Sigma}, \quad \mathrm{ULNM}_{X \times A_{K}^{n}[0,0], \Sigma}^{\prime} \stackrel{=}{\longrightarrow} \mathrm{ULNM}_{X \times A_{K}^{n}(I), \Sigma}^{\prime} .
$$

In particular, we have the canonical equivalence $\mathrm{ULNM}_{X \times A_{K}^{n}(I), \Sigma}=\mathrm{ULNM}_{X \times A_{K}^{n}(I), \Sigma}^{\prime}$ if $\prod_{i=r+1}^{r+n} \Sigma_{i} \subseteq K^{n}$.

Proof. It is immediate from Proposition 1.14 that the two functors are fully faithful. Moreover, since any $\Sigma$-unipotent object can be written as a successive extension by $\Sigma$-constant objects and since any $\Sigma$-constant object is in the essential image of $\mathcal{U}_{I}$, we see that the former functor is essentially surjective, by using Proposition 1.14 again.

Let us prove that the latter functor is also essentially surjective. Take an object $(E, \nabla)$ in $\mathrm{ULNM}_{X \times A_{K}^{n}(I), \Sigma}^{\prime}$. Then there exists a finite Galois extension $K \subseteq K^{\prime}$ such that the restriction $\left(E^{\prime}, \nabla^{\prime}\right)$ of $(E, \nabla)$ to $X_{K^{\prime}} \times A_{K^{\prime}}^{n}(I)$ (where $X_{K^{\prime}}$ is the rigid analytic space over $K^{\prime}$ naturally induced by $\left.X\right)$ is $\Sigma$-unipotent. Then, since the functor $\mathcal{U}_{I, K^{\prime}}$ : $\mathrm{ULNM}_{X_{K^{\prime}} \times A_{K^{\prime}}^{n}[0,0], \Sigma} \longrightarrow \mathrm{ULNM}_{X_{K^{\prime}} \times A_{K^{\prime}}^{n}(I), \Sigma}$ is an equivalence of categories, $\left(E^{\prime}, \nabla^{\prime}\right)$ comes from an object $\left(F^{\prime}, \nabla^{\prime}\right)$ in $\mathrm{ULNM}_{X_{K^{\prime}} \times A_{K^{\prime}}^{n}[0,0], \Sigma}$. Moreover, since $\left(E^{\prime}, \nabla^{\prime}\right)$ is 
endowed with descent data $\iota_{\sigma}: \sigma^{*}\left(E^{\prime}, \nabla^{\prime}\right) \stackrel{=}{\longrightarrow}\left(E^{\prime}, \nabla^{\prime}\right)\left(\sigma \in \operatorname{Gal}\left(K^{\prime} / K\right)\right),\left(F^{\prime}, \nabla^{\prime}\right)$ is also endowed with the descent data which is sent to $\left\{\iota_{\sigma}\right\}_{\sigma}$ by $\mathcal{U}_{I, K^{\prime}}$. Then $\left(F^{\prime}, \nabla^{\prime}\right)$ descends uniquely to an object $(F, \nabla)$ in $\operatorname{ULNM}_{X \times A_{K}^{n}[0,0], \Sigma}^{\prime}$ which is sent to $(E, \nabla)$ by $\mathcal{U}_{I}$. Hence the latter functor is also essentially surjective.

The last assertion follows from the two equivalences of categories and Remark $1.13(1)$.

Corollary 1.16 (cf. [10, 3.3.6]). Let $X, x_{1}, \ldots, x_{r}, \Sigma$ be as in Proposition 1.14 and let $I$ be an aligned interval of positive length. Then $\mathcal{U}_{I}$ induces fully-faithful functors $\mathrm{ULNM}_{X \times A_{K}^{n}[0,0], \Sigma}^{\left({ }^{\prime}\right)} \longrightarrow \mathrm{ULNM}_{X \times A_{K}^{n}(I), \Sigma}^{\left({ }^{\prime}\right)}$

Proof. It is easy to see that the functor $\mathcal{U}_{I}$ is faithful. On the other hand, if we take a quasi-open subinterval $J \subset I$ of positive length, we have the faithful restriction functor $\mathrm{ULNM}_{X \times A_{K}^{n}(I), \Sigma}^{\left({ }^{\prime}\right)} \longrightarrow \mathrm{ULNM}_{X \times A_{K}^{n}(J), \Sigma}^{\left({ }^{\prime}\right)}$ such that the composite functor

$$
\mathrm{ULNM}_{X \times A_{K}^{n}[0,0], \Sigma}^{\left({ }^{\prime}\right)} \stackrel{\mathcal{U}_{I}}{\longrightarrow} \operatorname{ULNM}_{X \times A_{K}^{n}(I), \Sigma}^{\left({ }^{\prime}\right)} \longrightarrow \mathrm{ULNM}_{X \times A_{K}^{n}(J), \Sigma}^{\left({ }^{\prime}\right)},
$$

which is nothing but $\mathcal{U}_{J}$, is an equivalence of categories by Corollary 1.15. From this, we see that the functor $\mathcal{U}_{I}$ is full.

The following proposition is the analogue of [10, 3.2.20].

Proposition 1.17. Let $X, x_{1}, \ldots, x_{r}, \Sigma$ is as in Proposition 1.14 and let I be a quasiopen subinterval or a closed aligned subinterval of positive length in $[0, \infty)$. Then $\mathrm{ULNM}_{X \times A_{K}^{n}(I), \Sigma}^{(')}$ is an abelian subcategory of $\mathrm{LNM}_{X \times A_{K}^{n}(I), \Sigma}$. Moreover, for any $E \in \mathrm{ULNM}_{X \times A_{K}^{n}(I), \Sigma}^{\left({ }^{\prime}\right)}$, any subquotient of $E$ in the category $\operatorname{LNM}_{X \times A_{K}^{n}(I), \Sigma}$ belongs to the category $\mathrm{ULNM}_{X \times A_{K}^{n}(I), \Sigma}^{\left({ }^{\prime}\right)}$.

Proof. It suffices to prove the latter assertion and it suffices to work only in the case of $\mathrm{ULNM}_{X \times A_{K}^{n}(I), \Sigma}$. Take $E \in \mathrm{ULNM}_{X \times A_{K}^{n}(I), \Sigma}$ and let $F$ be a subquotient of $E$ in the category $\operatorname{LNM}_{X \times A_{K}^{n}(I), \Sigma}$. First we prove the proposition in the case $I$ is quasi-open, assuming that the proposition is true in the closed aligned case. Write $I$ as the union $I=\bigcup_{m=1}^{\infty}\left[a_{m}, b_{m}\right]$ of closed aligned intervals. Then $\left.F\right|_{X \times A_{K}^{n}\left[a_{m}, b_{m}\right]}$ are $\Sigma$-unipotent by assumption. Then we can write $\left.F\right|_{X \times A_{K}^{n}\left(a_{m}, b_{m}\right)}=\mathcal{U}_{\left(a_{m}, b_{m}\right)}\left(G_{m}\right)$ for some $G_{m} \in \mathrm{LNM}_{X \times A_{K}^{n}[0,0], \Sigma}$, and the compatibility of $\left.F\right|_{X \times A_{K}^{n}\left(a_{m}, b_{m}\right)}$ 's with respect to $m$ implies that $G_{m}(m \in \mathbb{N})$ does not depend on $m$, which we denote by $G$. Then we have $\left.F\right|_{X \times A_{K}^{n}\left(a_{m}, b_{m}\right)}=\mathcal{U}_{\left(a_{m}, b_{m}\right)}(G)$ and hence $F=\mathcal{U}_{I}(G)$. Hence $F$ is $\Sigma$ unipotent, as desired. So it suffices to prove the proposition in the case where $I$ is a closed aligned interval of positive length.

To prove the proposition, it suffices to see that the subquotients of a $\Sigma$-constant object $E$ is again $\Sigma$-constant. Then it suffices to check one of the subobjects or the quotients are $\Sigma$-constant, by considering the dual and noting that $-\Sigma:=$ $\left\{\left(\xi_{i}\right) \mid \forall i,-\xi_{i} \in \Sigma_{i}\right\}$ is again (NID) and (NLD). 
First we prove that, for $n=1$ and $X=\operatorname{Spm} K(r=0)$, being $\Sigma$-constant is stable by taking quotients. Let $E$ be a $\Sigma$-constant object and let $f: E \longrightarrow E^{\prime}$ be a surjection in $\mathrm{LNM}_{A_{K}^{1}(I), \Sigma}$. Then, for some $\xi=\left(\xi_{1}, \ldots, \xi_{n}\right) \in \Sigma \cap K^{n}, E \otimes M_{-\xi}$ is $\{0\}^{n}$-constant, that is, constant in the sense of [10] and $f$ induces the surjection $E \otimes M_{-\xi} \longrightarrow E^{\prime} \otimes M_{-\xi}$ in $\operatorname{LNM}_{A_{K}^{1}(I),\{0\}^{n}}$. Let $F^{\prime}$ be the image of $\{x \in E \otimes$ $\left.M_{-\xi} \mid \forall i, t_{i} \frac{\partial}{\partial t_{i}}(x)=0\right\}$ in $E^{\prime} \otimes M_{-\xi}$. Then the map $F^{\prime} \otimes_{K} \mathcal{O}_{A_{K}^{n}(I)} \longrightarrow E^{\prime} \otimes M_{-\xi}$ is suejective by construction and injective by [10, 3.2.19]. Hence it is an isomorphism, that is, $E^{\prime} \otimes M_{-\xi}$ is $\{0\}^{n}$-constant. Hence $E^{\prime}$ is $\Sigma$-constant (more precisely, $\prod_{i=1}^{n}\left\{\xi_{i}\right\}$ constant).

Next we check that, for $n$ and $X$ arbitrary, being $\Sigma$-constant is stable by taking subobject. Let $E$ be a $\Sigma$-constant object and let us take a subobject $F \hookrightarrow E$ in $\mathrm{LNM}_{X \times A_{K}^{n}(I), \Sigma}$. First we reduce to the case $n=1$ and $X$ arbitrary. To do this, we put $Y:=X \times A_{K}^{n-1}(I)$. Then $E$ is $\Sigma$-constant on $Y \times A_{K}^{1}(I)$. By the proposition in the case $n=1$, we see that $F$ is also $\Sigma$-constant on $Y \times A_{K}^{1}(I)$. By definition of $\Sigma$-constance, we have $E=\mathcal{U}_{I}\left(E^{\prime}\right), F=\mathcal{U}_{I}\left(F^{\prime}\right)$ for some $E^{\prime}, F^{\prime} \in \operatorname{LNM}_{Y \times A_{K}^{1}[0,0], \Sigma}$. Then, by Corollary 1.16, the inclusion $F \hookrightarrow E$ comes from an inclusion $F^{\prime} \hookrightarrow E^{\prime}$. Let us express $E^{\prime}, F^{\prime}$ as $\left(E^{\prime \prime}, \partial_{E}\right),\left(F^{\prime \prime}, \partial_{F}\right)$, where $E^{\prime \prime}, F^{\prime \prime}$ are objects in $\operatorname{LNM}_{Y, \prod_{i=1}^{n-1} \Sigma_{i}}=$ $\mathrm{LNM}_{X \times A_{K}^{n-1}(I), \prod_{i=1}^{n-1} \Sigma_{i}}$ and $\partial_{E}, \partial_{F}$ are endomorphisms on $E^{\prime \prime}, F^{\prime \prime}$, respectively. Then $E^{\prime \prime}$ is $\prod_{i=1}^{r+n-1} \Sigma_{i}$-consant and $\partial_{E}$ is the multiplication by some $\xi \in \Sigma_{r+n} \cap K$. Then, $F^{\prime \prime}$ is also $\prod_{i=1}^{r+n-1} \Sigma_{i}$-constant by induction hypothesis and $\partial_{F}=\left.\partial_{E}\right|_{F}$ is also the multiplication by $\xi$. Hence $F^{\prime}=\left(F^{\prime \prime}, \partial_{F}\right)$ is $\Sigma$-constant and so $F=\mathcal{U}_{I}\left(F^{\prime}\right)$ is $\Sigma$-constant on $X \times A_{K}^{n}(I)$.

Finally we prove the claim in the previous paragraph in the case $n=1$. Let $F \hookrightarrow E$ be as above. Note first that, to prove the $\Sigma$-constance of $F$, we may replace $K$ by its finite Galois extension $K^{\prime}$ : Indeed, if we denote the restriction of $F$ to $X_{K^{\prime}} \times A_{K^{\prime}}^{1}(I)$ by $F_{K^{\prime}}$, it carries the descent data $\iota_{\sigma}: \sigma^{*} F_{K^{\prime}} \stackrel{=}{\longrightarrow} F_{K^{\prime}}(\sigma \in$ $\left.\operatorname{Gal}\left(K^{\prime} / K\right)\right)$. Then, if we have $F_{K^{\prime}}=\mathcal{U}_{I}\left(F_{0, K^{\prime}}\right)$ for some $\Sigma$-constant object $F_{0, K^{\prime}}$ on $X_{K^{\prime}} \times A_{K^{\prime}}^{1}[0,0], \iota_{\sigma}$ 's descend uniquely to the descent data on $F_{0, K^{\prime}}$, thanks to the full-faithfulness of $\mathcal{U}_{I}$ (Corollary 1.16). Hence $F_{0, K^{\prime}}$ descends to a $\Sigma$-constant object $F_{0}$ in $X_{K} \times A_{K}^{1}[0,0]$ satisfying $\mathcal{U}_{I}\left(F_{0}\right)=F$, as desired. So we may assume that $A_{K}^{1}(I)$ admits a $K$-rational point $x$. Now let us take $\xi \in \Sigma_{r+1}$ such that $E \otimes \pi^{*} M_{-\xi}$ is $\prod_{i=1}^{r} \Sigma_{i} \times\{0\}$-constant, where $\pi$ denotes the projection $X \times A_{K}^{1}(I) \longrightarrow X$. Denote the section $X \hookrightarrow X \times A_{K}^{1}(I)$ associated to $x$ also by $x$ and put $F^{\prime}:=\operatorname{Im}\left(x^{*}(F \otimes\right.$ $\left.\left.\pi^{*} M_{-\xi}\right) \longrightarrow x^{*}\left(E \otimes \pi^{*} M_{-\xi}\right)\right)$. Then we have

$$
\pi^{*} F^{\prime} \otimes M_{\xi}=\operatorname{Im}\left(\pi^{*} x^{*}\left(F \otimes \pi^{*} M_{-\xi}\right) \longrightarrow \pi^{*} x^{*}\left(E \otimes \pi^{*} M_{-\xi}\right)\right) \otimes M_{\xi} \hookrightarrow E .
$$

To prove the claim, it suffices to prove the equality $F=\pi^{*} F^{\prime} \otimes M_{\xi}$. Hence it suffices to prove that the composition maps $F \hookrightarrow E \longrightarrow E /\left(\pi^{*} F^{\prime} \otimes M_{\xi}\right)$ and $\pi^{*} F^{\prime} \otimes M_{\xi} \hookrightarrow$ $E \longrightarrow E / F$ are zero. To check this, we may replace $X$ by $X^{\prime}:=$ the complement of the zero loci of $x_{i}$ 's. (Then one can ignore the part ' $\prod_{i=1}^{r} \Sigma_{i}$ '.) Moreover, it suffices to check on a neighborhood of each point of $X^{\prime}$. Hence we may assume that $X^{\prime}$ is equal to a closed polydisc of radius $p^{-m}$ for some $m$ (after possibly enlarging $K$ ). 
Let $L$ be the completion of the fraction field of $\mathcal{O}\left(X^{\prime}\right)$ with respect to the spectrum norm on $\mathcal{O}\left(X^{\prime}\right)$. (This is possible because $\mathcal{O}\left(X^{\prime}\right)$ is a reduced affinoid algebra whose reduction is an integral domain.) Then it suffices to check the vanishing of the above two maps after we pull them back to $A_{L}^{1}(I)$. Then it is true because we already know that $F$ is $\{\xi\}$-constant on $A_{L}^{1}(I)$. So we proved the claim and hence the proof of the proposition is finished.

Using Corollary 1.15 and Proposition 1.17, we can prove the following, which is an analogue of [10, 3.3.8]:

Proposition 1.18. Let $X$ be a smooth rigid space endowed with $x_{1}, \ldots, x_{r} \in \Gamma\left(X, \mathcal{O}_{X}\right)$ whose zero loci are smooth and meet transversally and Let $U$ be the complement of these zero loci. Let $\Sigma=\prod_{i=1}^{r} \Sigma_{i}$ be a subset of $\bar{K}^{r}$ which is (NID) and (NLD). Let $E$ be an object in $\operatorname{LNM}_{X, \Sigma}$ and let $F$ be a sub $\nabla$-module of the restriction of $E$ to $U$. Then $F$ uniquely extends to a subobject $\widetilde{F}$ of $E$ in the category $\operatorname{LNM}_{X, \Sigma}$.

Proof. The proof is the same as [10, 3.3.8]. By induction, it suffices to check the following claim: Let $Z$ be the zero locus of $t_{r}$ and suppose that we are given a sub

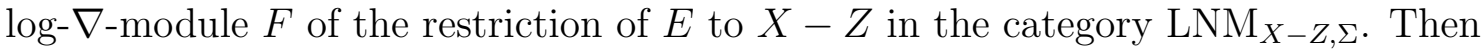
$F$ uniquely extends to a subobject $\widetilde{F}$ of $E$ in the category $\operatorname{LNM}_{X, \Sigma}$.

Since the claim is local, we may assume that $Z, X$ are affinoid. Then, by [12, 1.18], we may assume moreover that $X$ has the form $Z \times A_{K}^{1}[0, a]$ for some $a \in$ $(0, \infty) \cap \Gamma^{*}$ and that the restriction of $E$ to $Z$ is free. Also, we may shrink $X$ in order that $X=Z \times A_{K}^{1}[0, a)$ with $a$ replaced by a smaller positive value. So, by Lemma 1.9 and Remark 1.13, we may assume that $E$ is potentially $\Sigma$-unipotent and $E$ has the form $\mathcal{U}_{[0, a)}(G)$ for some $G \in \operatorname{LNM}_{Z \times A_{K}^{1}[0,0], \Sigma}$. Since $F$ is a subobject of $\left.E\right|_{Z \times A_{K}^{1}(0, a)}$ in the category $\operatorname{LNM}_{Z \times A_{K}^{1}(0, a), \Sigma}$ and $\left.E\right|_{Z \times A_{K}^{1}(0, a)}$ is potentially $\Sigma$ unipotent, we see by Proposition 1.17 that $F$ is also potentially $\Sigma$-unipotent, that is, $F$ has the form $\mathcal{U}_{(0, a)}(H)$ for some $H \in \operatorname{LNM}_{Z \times A_{K}^{1}[0,0], \Sigma}$. By Corollary 1.15, we see that the inclusion $\left.F \hookrightarrow E\right|_{Z \times A_{K}^{1}(0, a)}$ is induced by some injection $H \hookrightarrow G$ in $\operatorname{LNM}_{Z \times A_{K}^{1}[0,0], \Sigma}$. Then, if we put $\widetilde{F}:=\mathcal{U}_{[0, a)}(H)$, it defines a sub $\log -\nabla$-module of $E$ which extends $F$. To see the uniqueness of the extension, we may work locally as above. Hence we may assume that the extensions should be potentially $\Sigma$-unipotent and then the uniqueness follows from Corollary 1.15.

\section{L-unipotence, generization and log-convergence}

In this section, we prove three key propositions in this paper. The first one, called generization, asserts that the property of $\Sigma$-unipotence is 'generic on the base'. It is an analogue of [10, 3.4.1, 3.4.3]. The second one, called overconvergent generization, asserts that one can extend the the property of $\Sigma$-unipotence 'overconvergently on the base'. It is an analogue of [10, 3.5.3]. The third one asserts that, under the condition of log-convergence, the property of 'having exponents in $\Sigma$ ' implies the 
$\Sigma$-constance. It is an analogue of [10, 3.6.2, 3.6.9] and a variant of [2, 6.5.2] (see also [1, [5]).

Here we explain the slight difference of the results in this section from the corresponding ones in the paper [10]. It seems to us that there is an error in the proof of [10, 3.5.3] and [10, 3.6.2]. (See Remark 2.8 for detail.) By this reason and some technical reason, our analogues corresponding to them are slightly weaker than the results in [10] when restricted to the unipotent case. On the other hand, our analogue concerning generization is slightly more generalized than in [10], since we need a slightly generalized version to prove the main result in the next section.

First we recall the notion concerning the convergence of multisequences and introduce a notation:

Definition 2.1 ([10, 2.4.1]). For an affinoid space $X$, a coherent $\mathcal{O}_{X}$-module $E$ and $\eta \in[0, \infty)$, a multisequence $\left\{\mathbf{v}_{I}\right\}_{I}$ of elements in $\Gamma(X, E)$ indexed by $I=$ $\left(i_{1}, \ldots, i_{n}\right) \in \mathbb{N}^{n}$ is called $\eta$-null if, for any multisequence $\left\{c_{I}\right\}_{I}$ in $K$ with $\left|c_{I}\right| \leq \eta^{|I|}$ (where $\left.|I|:=\sum_{j=1}^{n} i_{j}\right)$, the multisequence $\left\{c_{I} \mathbf{v}_{I}\right\}_{I}$ converges to zero in $\Gamma(X, E)$ with respect to the topology induced by the affinoid topology on $X$.

Definition 2.2. Let $X$ be an affinoid rigid space endowed with sections $x_{1}, \ldots, x_{r} \in$ $\Gamma\left(X, \mathcal{O}_{X}\right)$, let $I \subseteq[0, \infty)$ be a closed aligned subinterval and let $E$ be a log- $\nabla$-module on $X \times A_{K}^{n}(I)$. Then, for $\xi:=\left(\xi_{1}, \ldots, \xi_{n}\right) \in K^{n}$, we define $H_{X, \xi}^{0}\left(X \times A_{K}^{n}(I), E\right)$ by

$$
H_{X, \xi}^{0}\left(X \times A_{K}^{n}(I), E\right):=\left\{\mathbf{e} \in \Gamma\left(X \times A_{K}^{n}(I), E\right) \mid \forall i,\left(t_{i} \frac{\partial}{\partial t_{i}}-\xi_{i}\right)(\mathbf{e})=0\right\} .
$$

Then the key lemma for the generization is the following (this is the analogue of $[10,3.4 .1])$ :

Lemma 2.3. Let $A$ be an affinoid algebra such that $X:=\operatorname{Spm} A$ is smooth and endowed with $x_{1}, \ldots, x_{r} \in \Gamma\left(X, \mathcal{O}_{X}\right)$ whose zero loci are smooth and meet transversally and let $A \subseteq L$ be one of the following:

(1) $L$ is an affinoid algebra over $K$ satisfying the following conditions: $\operatorname{Spm} L$ is smooth, the zero loci of $x_{1}, \ldots, x_{r}$ are smooth and meet transversally on $\operatorname{Spm} L$ and the supremum norm on $L$ restricts to the supremum norm on $A$.

(2) $L$ is a field containing $A$ which is complete for a norm restricting to the supremum norm on $A$.

Let $I$ be a quasi-open subinterval of positive length in $[0,1)$ and let us write $\operatorname{Spm} L \times$ $A_{K}^{n}(I)$ simply by $A_{L}^{n}(I)$ in the case (1). (In the case $(2), A_{L}^{n}(I)$ is already defined as polyannulus over L.) Let $\Sigma:=\Sigma^{\prime} \times \prod_{i=1}^{n} \Sigma_{i}$ be a subset of $\bar{K}^{r} \times \mathbb{Z}_{p}^{n}$ which is (NID) and (NLD) and let $E$ be an object in $\mathrm{LNM}_{X \times A_{K}^{n}(I), \Sigma}$ such that the induced object $F \in \mathrm{LNM}_{A_{L}^{n}(I), \Sigma}$ is $\Sigma$-unipotent. Then, for any closed aligned subinterval $[b, c]$ of $I$, there exists an element $\xi \in \prod_{i=1}^{n} \Sigma_{i}$ such that $H_{X, \xi}^{0}\left(X \times A_{K}^{n}(I), E\right) \neq 0$.

Proof. Since $F$ is $\Sigma$-unipotent, we have $F=\mathcal{U}_{I}(W)$ for some $W \in \operatorname{LNM}_{A_{L}^{n}[0,0], \Sigma}$ and $W$ is regarded as a $\log -\nabla$-module on $\operatorname{Spm} L$ with respect to $x_{1}, \ldots, x_{r}$ (resp. a finite 
dimensional $L$-vector space) endowed with commuting endomorphisms $N_{i}:=t_{i} \frac{\partial}{\partial t_{i}}$ with eigenvalues in $\Sigma_{i}(1 \leq i \leq n)$ in the case (1) (resp. (2)). Let $\xi_{i k} \in \Sigma_{i}$ $\left(1 \leq k \leq n_{i}\right)$ be the eigenvalues of $N_{i}$ and let $P_{i}(x)=\prod_{k=1}^{n_{i}}\left(x-\xi_{i k}\right)^{m_{i k}} \in K[x]$ be the minimal monic polynomial satisfying $P_{i}\left(N_{i}\right)=0$. Let us put $m:=\max _{i, k}\left(m_{i k}\right)$. For $\mathbf{k}:=\left(k_{1}, \ldots, k_{n}\right)\left(1 \leq k_{i} \leq n_{i}\right)$, let us define

$$
\begin{aligned}
& W_{\mathbf{k}}:=\left\{x \in W \mid \forall i,\left(N_{i}-\xi_{i k_{i}}\right)^{m}(x)=0\right\}, \\
& W_{\mathbf{k}}^{\prime}:=\left\{x \in W \mid \forall i,\left(N_{i}-\xi_{i k_{i}}\right)(x)=0\right\} .
\end{aligned}
$$

Then, for some $\mathbf{k}, W_{\mathbf{k}}^{\prime}$ is non-zero. Hence we may assume that $W_{\mathbf{1}}^{\prime}$ (where $\mathbf{1}:=$ $(1, \ldots, 1))$ is non-zero. Then there exist polynomials $Q_{i}(x)$ dividing $P_{i}(x)(1 \leq i \leq n)$ such that the map $\prod_{i=1}^{n} Q\left(N_{i}\right): W \longrightarrow W$ is a non-zero map whose image is contained in $W_{\mathbf{1}}^{\prime}$. Also, there exist $L$-linear maps $\varphi_{\mathbf{k}}: W \longrightarrow W\left(\mathbf{k}=\left(k_{1}, \ldots, k_{n}\right)\right.$, $\left.1 \leq k_{i} \leq n_{i}\right)$ whose image is contained in $W_{\mathbf{k}}$ such that $\sum_{\mathbf{k}} \varphi_{\mathbf{k}}$ is equal to the identity map on $W$.

Now define the sequence of operators $D_{l}(l \in \mathbb{N})$ on $E$ by

$$
D_{l}:=\prod_{i=1}^{n}\left\{Q_{i}\left(t_{i} \frac{\partial}{\partial t_{i}}\right) \prod_{k=1}^{n_{i}} \prod_{j=1}^{l}\left(\frac{j-\left(t_{i} \frac{\partial}{\partial t_{i}}-\xi_{i k}\right)}{j-\left(\xi_{i 1}-\xi_{i k}\right)} \cdot \frac{j+\left(t_{i} \frac{\partial}{\partial t_{i}}-\xi_{i k}\right)}{j+\left(\xi_{i 1}-\xi_{i k}\right)}\right)^{m}\right\} .
$$

Take an aligned closed subinterval $[d, e]$ in $I$ with $d<b, c<e$ if $b>0$ and $d=0, c<e$ if $b=0$. We prove the following claim:

claim 1. For any $\mathbf{v} \in \Gamma\left(X \times A_{K}^{n}[d, e], E\right)$, the sequence $\left\{D_{l}(\mathbf{v})\right\}_{l}$ converges to an element in $H_{X, \xi}^{0}\left(X \times A_{K}^{n}[b, c], E\right)$, where $\xi:=\left(\xi_{11}, \ldots, \xi_{n 1}\right)$.

To prove the convergence, it suffice to check in $\Gamma\left(A_{L}^{n}[b, c], F\right)$. So we can write $\mathbf{v}$ as $\mathbf{v}:=\sum_{J} t_{1}^{j_{1}} \cdots t_{n}^{j_{n}} \mathbf{v}_{J}$ with $\mathbf{v}_{J} \in W$. Let us put $\mathbf{v}_{J, \mathbf{k}}:=\varphi_{\mathbf{k}}\left(\mathbf{v}_{J}\right)$. Then we have

$$
\begin{aligned}
D_{l}(\mathbf{v}) & =\sum_{\mathbf{k}}\left\{\sum_{J} t_{1}^{j_{1}} \cdots t_{n}^{j_{n}} .\right. \\
& \left.\prod_{i=1}^{n}\left\{Q_{i}\left(N_{i}+j_{i}\right) \prod_{k=1}^{n_{i}} \prod_{j=1}^{l}\left(\frac{j-\left(N_{i}+j_{i}-\xi_{i k}\right)}{j-\left(\xi_{i 1}-\xi_{i k}\right)} \cdot \frac{j+\left(N_{i}+j_{i}-\xi_{i k}\right)}{j+\left(\xi_{i 1}-\xi_{i k}\right)}\right)^{m}\right\}\left(\mathbf{v}_{J, \mathbf{k}}\right)\right\} .
\end{aligned}
$$

We compute the term

$$
\prod_{i=1}^{n}\left\{Q_{i}\left(N_{i}+j_{i}\right) \prod_{k=1}^{n_{i}} \prod_{j=1}^{l}\left(\frac{j-\left(N_{i}+j_{i}-\xi_{i k}\right)}{j-\left(\xi_{i 1}-\xi_{i k}\right)} \cdot \frac{j+\left(N_{i}+j_{i}-\xi_{i k}\right)}{j+\left(\xi_{i 1}-\xi_{i k}\right)}\right)^{m}\right\}\left(\mathbf{v}_{J, \mathbf{k}}\right)
$$

for each $J, \mathbf{k}$. First, in the case $J=\mathbf{0}:=(0, \ldots, 0)$, we have $\prod_{i=1}^{n} Q\left(N_{i}\right)\left(\mathbf{v}_{\mathbf{0}, \mathbf{k}}\right) \in W_{\mathbf{1}}^{\prime}$. Hence the term (2.1) is equal to

$$
\prod_{i=1}^{n} \prod_{k=1}^{n_{i}} \prod_{j=1}^{l}\left(\frac{j-\left(\xi_{i 1}-\xi_{i k}\right)}{j-\left(\xi_{i 1}-\xi_{i k}\right)} \cdot \frac{j+\left(\xi_{i 1}-\xi_{i k}\right)}{j+\left(\xi_{i 1}-\xi_{i k}\right)}\right)^{m}\left(\prod_{i=1}^{n} Q\left(N_{i}\right)\left(\mathbf{v}_{\mathbf{0}, \mathbf{k}}\right)\right)=\prod_{i=1}^{n} Q\left(N_{i}\right)\left(\mathbf{v}_{\mathbf{0}, \mathbf{k}}\right) \text {. }
$$


Second, let us consider the case where $J:=\left(j_{1}, \ldots, j_{n}\right)$ contains some factor $j_{i}$ with $\left|j_{i}\right| \leq l$. If we put $\mathbf{k}:=\left(k_{1}, \ldots, k_{n}\right)$, then the term (2.1) contains a factor

$$
\left(\frac{j_{i}-\left(N_{i}+j_{i}-\xi_{i k_{i}}\right)}{j_{i}-\left(\xi_{i 1}-\xi_{j k_{i}}\right)}\right)^{m}=(\text { const }) \cdot\left(N_{i}-\xi_{i k_{i}}\right)^{m}
$$

or a factor

$$
\left(\frac{-j_{i}+\left(N_{i}+j_{i}-\xi_{i k_{i}}\right)}{-j_{i}+\left(\xi_{i 1}-\xi_{j k_{i}}\right)}\right)^{m}=(\mathrm{const}) \cdot\left(N_{i}-\xi_{i k_{i}}\right)^{m} .
$$

Hence we can conclude that the term (2.1) is equal to zero in this case. Finally, let us consider the case where any factor $j_{i}$ of $J:=\left(j_{1}, \ldots, j_{n}\right)$ satisfies $\left|j_{i}\right|>l$. In this case, let us rewrite the term (2.1) as

$$
\begin{aligned}
\prod_{i=1}^{n}\left\{Q_{i}\left(\left(N_{i}-\xi_{i k_{i}}\right)+\left(\xi_{i k_{i}}+j_{i}\right)\right)\right. & \prod_{k=1}^{n_{i}} \prod_{j=1}^{l}\left(\frac{\left(j-j_{i}+\xi_{i k}+\xi_{i k_{i}}\right)-\left(N_{i}-\xi_{i k_{i}}\right)}{j-\left(\xi_{i 1}-\xi_{i k}\right)}\right. \\
& \left.\left.\frac{\left(j+j_{i}-\xi_{i k}+\xi_{i k_{i}}\right)+\left(N_{i}-\xi_{i k_{i}}\right)}{j+\left(\xi_{i 1}-\xi_{i k}\right)}\right)^{m}\right\}\left(\mathbf{v}_{J, \mathbf{k}}\right) .
\end{aligned}
$$

Then we see that the operator in (2.2) between parentheses $\{\quad\}$ is a polynomial on $N_{i}-\xi_{i k_{i}}$ and hence it can be written as the form $\sum_{\alpha=0}^{m-1} c_{i, \alpha}^{j_{i}, l}\left(N_{i}-\xi_{i k_{i}}\right)^{\alpha}$ for some $c_{i, \alpha}^{j_{i}, l} \in K$ modulo $\left(N_{i}-\xi_{i k_{i}}\right)^{m}$. Then the term (2.1) can be expressed as

$$
\sum_{\alpha_{1}, \ldots, \alpha_{n}=0}^{m-1} \prod_{i=1}^{n}\left\{c_{i, \alpha_{i}}^{j_{i}, l}\left(N_{i}-\xi_{i k_{i}}\right)^{\alpha_{i}}\right\}\left(\mathbf{v}_{J, \mathbf{k}}\right)
$$

Therefore, we can calculate $D_{l}(\mathbf{v})-\prod_{i=1}^{n} Q_{i}\left(N_{i}\right)\left(\mathbf{v}_{\mathbf{0}}\right)$ as follows:

$$
\begin{aligned}
& D_{l}(\mathbf{v})-\prod_{i=1}^{n} Q_{i}\left(N_{i}\right)\left(\mathbf{v}_{\mathbf{0}}\right) \\
= & \sum_{\mathbf{k}} \sum_{\alpha_{1}, \ldots, \alpha_{n}=0}^{m-1} \sum_{\substack{J \\
\forall i,\left|j_{i}\right|>l}}\left(\prod_{i=1}^{n} c_{i, \alpha_{i}}^{j_{i}, l}\right) t_{1}^{j_{1}} \cdots t_{n}^{j_{n}} \cdot \prod_{i=1}^{n}\left(N_{i}-\xi_{i k_{i}}\right)^{\alpha_{i}}\left(\mathbf{v}_{J, \mathbf{k}}\right) .
\end{aligned}
$$

Since $\prod_{i=1}^{n} Q_{i}\left(N_{i}\right)\left(\mathbf{v}_{\mathbf{0}}\right)$ is killed by $N_{i}-\xi_{i 1}$ for any $i$, the proof of claim 1 is reduced to the following claim:

claim 2. The sequence $\left\{D_{l}(\mathbf{v})-\prod_{i=0}^{n} Q_{i}\left(N_{i}\right)\left(\mathbf{v}_{\mathbf{0}}\right)\right\}$ is $\eta$-null for some $\eta>1$.

By (2.3) and [10, 3.1.11] (see also the proof of [10, 3.4.1]), the claim 2 is reduced to the following: 
claim 3. There exists a constant $A$ such that, for any $\delta>1$, we have the inequality $\left|c_{i, \alpha}^{j^{\prime}, l}\right| \leq$ (const) $\delta^{A\left|j^{\prime}\right|}$ for all $i, \alpha$ and $j^{\prime}$ with $\left|j^{\prime}\right|>l$.

We prove claim 3. Let us define $p_{i, \alpha}^{j^{\prime}}, q_{i, k, \alpha}^{j^{\prime}, l}, r_{i, k, \alpha}^{j^{\prime}, l} \in K$ by

$$
\begin{aligned}
& Q_{i}\left(x-\left(\xi_{i k_{i}}+j^{\prime}\right)\right)=\sum_{\alpha=0}^{m-1} p_{i, \alpha}^{j^{\prime}} x^{\alpha} \bmod x^{m}, \\
& \prod_{j=1}^{l} \frac{\left(j-j^{\prime}+\xi_{i k}-\xi_{i k_{i}}\right)-x}{j-\left(\xi_{i 1}-\xi_{i k}\right)}=\sum_{\alpha=0}^{m-1} q_{i, k, \alpha}^{j^{\prime}, l} x^{\alpha} \bmod x^{m}, \\
& \prod_{j=1}^{l} \frac{\left(j+j^{\prime}-\xi_{i k}+\xi_{i k_{i}}\right)-x}{j+\left(\xi_{i 1}-\xi_{i k}\right)}=\sum_{\alpha=0}^{m-1} r_{i, k, \alpha}^{j^{\prime}, l} x^{\alpha} \quad \bmod x^{m} .
\end{aligned}
$$

Then, to prove the claim 3 , it suffices to prove the same assertion for $p_{i, \alpha}^{j^{\prime}}, q_{i, k, \alpha}^{j^{\prime}, l}$ and $r_{i, k, \alpha}^{j^{\prime}, l}\left(1 \leq k \leq n_{i}\right)$. Since $\xi_{i k_{i}}+j^{\prime}$ is in $\mathbb{Z}_{p},\left|p_{i, \alpha}^{j^{\prime}}\right|$ is equal or less than the maximum of the absolute values of the coefficients of $Q_{i}$ 's, which is independent of $i, \alpha$ and $j^{\prime}$. Hence the claim 3 is true for $\left|p_{i, \alpha}^{j^{\prime}}\right|$. Next, let us note that $\pm\left(\xi_{i 1}-\xi_{i k}\right), \pm\left(\xi_{i k}-\xi_{i k_{i}}\right)$ are in $\mathbb{Z}_{p}-(\mathbb{Z}-\{0\})$ and $p$-adically non-Liouville. Hence the claim 3 for $q_{i, k, \alpha}^{j^{\prime}, l}$ and $r_{i, k, \alpha}^{j^{\prime}, l}$ are reduced to the following claim:

claim 4. Let $\sigma, \tau \in \mathbb{Z}_{p}-(\mathbb{Z}-\{0\})$ be $p$-adically non-Liouville numbers and define $s_{\alpha}^{j^{\prime}, l}$ by

$$
\prod_{j=1}^{l} \frac{\left(j+j^{\prime}+\tau\right)+x}{j+\sigma}=\sum_{\alpha=0}^{m-1} s_{\alpha}^{j^{\prime}, l} x^{\alpha} \quad \bmod x^{m} .
$$

Then, for any $\delta>1$, we have $\left|s_{\alpha}^{j^{\prime}, l}\right| \leq$ (const) $\delta^{(2 m-1)\left|j^{\prime}\right|}$ for any $\alpha$ and $j^{\prime}$ with $\left|j^{\prime}\right|>l$.

We prove this claim. Let us fix $\delta>1$. Then by the $p$-adic non-Liouvilleness of $\tau$, we have, for some constant $C>1$, the inequalities

$$
\left|j+j^{\prime}+\tau\right|^{-1}<C \delta^{\left|j+j^{\prime}\right|}<C \delta^{2\left|j^{\prime}\right|}
$$

for any $j \leq l<\left|j^{\prime}\right|$. Hence we have

$$
\left|s_{\alpha}^{j^{\prime}, l}\right| \leq C^{\alpha} \delta^{2\left|j^{\prime}\right| \alpha}\left|s_{0}^{j^{\prime}, l}\right| \leq C^{m-1} \delta^{2(m-1)\left|j^{\prime}\right|}\left|s_{0}^{j^{\prime}, l}\right|
$$

for any $\alpha$ and $j^{\prime}$ with $\left|j^{\prime}\right|>l$. So it suffices to prove the inequality $\left|s_{0}^{j^{\prime}, l}\right| \leq$ (const) $\delta^{l}$ for any $j^{\prime}$ with $\left|j^{\prime}\right|>l$.

Note that we have $s_{0}^{j^{\prime}, l}=\prod_{j=1}^{l} \frac{j+j^{\prime}+\tau}{j+\sigma}=\prod_{j=1}^{l} \frac{j+j^{\prime}+\tau}{j} \cdot \prod_{j=1}^{l} \frac{j}{j+\sigma}$, and it is easy to see the inequality $\left|\prod_{j=1}^{l} \frac{j+j^{\prime}+\tau}{j}\right| \leq 1$. Moreover, the radius of 
convergence of the series $\sum_{l=0}^{\infty} \frac{X^{l}}{\prod_{j=1}^{l}(j+\sigma)}$ is equal to $p^{-1 /(p-1)}$ by [9, VI Lemma 1.2] and for any $\delta>1$, we have $\left|\prod_{j=1}^{l} j\right| \leq$ (const) $\left(\delta p^{-1 /(p-1)}\right)^{l}$. Therefore, for any $\delta>1$, we have $\left|\prod_{j=1}^{l} \frac{j}{j+\sigma}\right|<$ (const) $\delta^{l}$. So we obtain the inequality $\left|s_{0}^{j^{\prime}, l}\right| \leq$ (const) $\delta^{l}$ and hence we have proved the claim 4 . So the proof of the claims 1,2 and 3 are also finished.

By the claim, we can define, for any $\mathbf{v} \in \Gamma\left(X \times A_{K}^{n}[d, e], E\right)$ the element $f(\mathbf{v}):=\lim _{l} D_{i}(\mathbf{v}) \in H_{X, \xi}^{0}\left(X \times A_{K}^{n}[b, c], E\right)$ and it is equal to $\prod_{i=1}^{n} Q_{i}\left(N_{i}\right)\left(\mathbf{v}_{\mathbf{0}}\right)$. If we take a generator $\left\{\mathbf{w}_{1}, \ldots, \mathbf{w}_{s}\right\}$ of $\Gamma\left(X \times A_{K}^{n}[d, e], E\right)$, we see that the $L$-span of the set $\left\{t^{J} \mathbf{w}_{i} \mid J \in \mathbb{Z}^{n}, 1 \leq i \leq s\right\}$ (resp. $\left\{t^{J} \mathbf{w}_{i} \mid J \in \mathbb{N}^{n}, 1 \leq i \leq s\right\}$ ) is dense in $\Gamma\left(A_{L}^{n}[b, c], F\right)$ when $b>0$ (resp. $\left.b=0\right)$. Hence the $L$-span on the image of this set by $f$ is dense in $\prod_{i=1}^{n} Q_{i}\left(N_{i}\right)(W)$, which is non-zero. Hence there exists an element $\mathbf{v} \in \Gamma\left(X \times A_{K}^{n}[d, e], E\right)$ with $f(\mathbf{v}) \neq 0$. Hence we have $H_{X, \xi}^{0}\left(X \times A_{K}^{n}[b, c], E\right) \neq 0$, as desired.

By using Lemma 2.3, we can prove the following proposition, which is the analogue of [10, 3.4.3]:

Proposition 2.4 (generization). Let $A$ be an affinoid algebra such that $X:=\operatorname{Spm} A$ is smooth and endowed with $x_{1}, \ldots, x_{r} \in \Gamma\left(X, \mathcal{O}_{X}\right)$ whose zero loci are smooth and meet transversally and let $A \subseteq L$ be one of the following:

(1) $L$ is an affinoid algebra over $K$ such that $\operatorname{Spm} L$ is smooth, $x_{i}$ 's are invertible in $L$ and that the supremum norm on $L$ restricts to the supremum norm on $A$.

(2) $L$ is a field containing $A$ which is complete for a norm restricting to the supremum norm on $A$.

Let $I$ be a quasi-open subinterval of positive length in $[0,1)$ and let us define $A_{L}^{n}(I)$ as in Lemma 2.3. Let $\Sigma:=\Sigma^{\prime} \times \prod_{i=1}^{n} \Sigma_{i}$ be a subset of $\bar{K}^{r} \times \mathbb{Z}_{p}^{n}$ which is (NID) and (NLD), and let $E$ be an object in $\mathrm{LNM}_{X \times A_{K}^{n}(I), \Sigma}$ such that the induced object $F \in \mathrm{LNM}_{A_{L}^{n}(I), \Sigma}$ is $\Sigma$-unipotent. Then $E$ is also $\Sigma$-unipotent.

Proof. The proof is similar to that in [10, 3.4.3], although we provide some supplementary argument which was missing there.

Fix any closed aligned subinterval $[b, c] \subseteq I$ of positive length. Then, by Lemma 2.3 , there exists some $\xi:=\left(\xi_{1}, \ldots, \xi_{n}\right) \in \prod_{i=1}^{n} \Sigma_{i}$ such that $H_{E}:=H_{X, \xi}^{0}(X \times$ $\left.A_{K}^{n}[b, c], E\right) \neq 0$. First we prove the following claim:

claim 1. $H_{E}$ is a finitely generated $A$-module and the canonical map $\pi_{X}^{*} H_{E} \longrightarrow E$ (where $\pi_{X}: X \times A_{K}^{n}[b, c] \longrightarrow X$ is the projection) is injective.

Let us take any finitely generated sub $A$-module $M \subseteq H_{E}$ and consider the fol- 
lowing diagram:

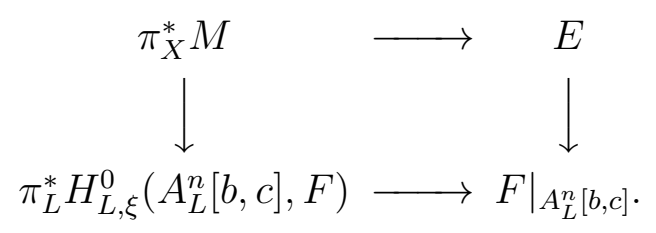

Then the vertical arrows are injective. We prove that the lower horizontal arrow is also injective: Indeed, if we write $\left.F\right|_{A_{L}^{n}[b, c]}$ by $\left.F\right|_{A_{L}^{n}[b, c]}=\mathcal{U}_{[b, c]}(W)$ as in the proof of Lemma 2.3, a formal power series computation shows the equality $H_{L, \xi}^{0}\left(A_{L}^{n}[b, c], F\right)=$ $\left\{x \in W \mid \forall i, N_{i}(x)=\xi_{i} x\right\}$. (Here $N_{i}$ is as in the proof of Lemma 2.3.) Hence we have the injection $H_{L, \xi}^{0}\left(A_{L}^{n}[b, c], F\right) \hookrightarrow W$ which induces the desired injection $\pi_{L}^{*} H_{L, \xi}^{0}\left(A_{L}^{n}[b, c], F\right) \hookrightarrow \pi_{L}^{*} W=\left.F\right|_{A_{L}^{n}[b, c]}$. So, by the diagram (2.4), we see that the map $\pi_{X}^{*} M \longrightarrow E$ is injective for all finitely generated sub $A$-module $M$ of $H_{E}$. By the Noetherianness of $E$, we can choose $M$ so that the image of $\pi_{X}^{*} M$ in $E$ is maximal among the modules of this form. Then, if $M$ is strictly smaller than $H_{E}$, we can choose a finitely generated sub $A$-module $M^{\prime}$ of $H_{E}$ which strictly contains $M$ and then we have a strict injection $\pi_{X}^{*} M \subsetneq \pi_{X}^{*} M^{\prime}$, which contradicts the definition of $M$. Hence we have $M=H_{E}$. So $H_{E}$ is a finitely generated $A$-module and the $\operatorname{map} \pi_{X}^{*} H_{E} \longrightarrow E$ is injective, as desired.

By claim 1, we can naturally regard $H_{E}$ as a coherent $\mathcal{O}_{X}$-module. Let $X^{\prime}$ be the complement of the zero loci of $x_{1}, \ldots, x_{r}$ in $X$ and let us put $G^{\prime}:=\pi_{X^{\prime}}^{*}\left(\left.H_{E}\right|_{X^{\prime}}\right.$ ) (where $\pi_{X^{\prime}}$ denotes the restriction of $\pi_{X}$ to $X^{\prime} \times A_{K}^{n}[b, c]$ ), which is a coherent submodule of $E^{\prime}:=\left.E\right|_{X^{\prime} \times A_{K}^{n}[b, c]}$. Then we prove the following claim:

claim 2. $\quad G^{\prime}$ has a natural structure of a $\Sigma$-constant log- $\nabla$-module on $X^{\prime} \times A_{K}^{n}[b, c]$ which is a subobject of $E^{\prime}$ in $\mathrm{LNM}_{X^{\prime} \times A_{K}^{n}[b, c], \Sigma}$.

Indeed, we see easily that $\left.H_{E}\right|_{X^{\prime}} \subseteq E^{\prime}$ is stable by continuous derivations of $X^{\prime}$ over $K$ and it admits commuting endomorphisms $t_{i}\left(\partial / \partial t_{i}\right)(1 \leq i \leq n)$, which is nothing but the multiplication by $\xi_{i}$. If we forget the endomorphisms $t_{i}\left(\partial / \partial t_{i}\right)$, $\left.H_{E}\right|_{X^{\prime}}$ is regarded as a $\nabla$-module on $X^{\prime}$. Hence it is a locally free $\mathcal{O}_{X^{\prime}}$-module. Then, adding the endomorphisms $t_{i}\left(\partial / \partial t_{i}\right)(1 \leq i \leq n),\left.H_{E}\right|_{X^{\prime}}$ is regarded as an object in $\mathrm{LNM}_{X^{\prime} \times A_{K}^{n}[0,0], \Sigma}$. Then $G^{\prime}=\pi_{X^{\prime}}^{*}\left(\left.H_{E}\right|_{X^{\prime}}\right) \hookrightarrow E^{\prime}$ is naturally regarded as $\mathcal{U}_{[b, c]}\left(\left.H_{E}\right|_{X^{\prime}}\right)$. Hence $G^{\prime}$ is $\Sigma$-constant (in fact $\Sigma^{\prime} \times \prod_{i=1}^{n}\left\{\xi_{i}\right\}$-constant), as desired.

Now let $G \subseteq E$ be the subobject in the category $\operatorname{LNM}_{X \times A_{K}^{n}[b, c], \Sigma}$ extending $G^{\prime} \subseteq E^{\prime}$, which uniquely exists by Proposition 1.18. Also, we put $H_{G}:=H_{X, \xi}^{0}(X \times$ $\left.A_{K}^{n}[b, c], G\right) \subseteq H_{E}$. (Then, by the same argument as the proof of claim 1 , we see that $H_{G}$ is also coherent.) Next we prove the following claim:

claim 3. The canonical map $\pi_{X}^{*} H_{G} \longrightarrow G$ is surjective.

Let us note that the restriction $G_{L}$ of $G$ to $A_{L}^{n}[b, c]$, which is equal to the restriction of $G^{\prime}=\mathcal{U}_{[b, c]}\left(\left.H_{E}\right|_{X^{\prime}}\right)$ to $A_{L}^{n}[b, c]$, is $\Sigma^{\prime} \times \prod_{i=1}^{n}\left\{\xi_{i}\right\}$-constant. Hence, if we take 
$[d, e] \supseteq[b, c]$ as in Lemma 2.3, we can define the map

$$
f=\lim _{l} D_{l}: \Gamma\left(X \times A_{K}^{n}[d, e], G\right) \longrightarrow H_{X, \xi}^{0}\left(X \times A_{K}^{n}[b, c], G\right)=H_{G}
$$

as in Lemma 2.3 with $Q_{i}(x)=1(1 \leq i \leq n)$ in the definition of $D_{l}$. Then the calculation in Lemma 2.3 says that, if we write $G_{L}=\mathcal{U}_{[b, c]}(V)\left(V \in \operatorname{LNM}_{A_{L}^{n}[0,0], \Sigma}\right)$ and if we express an element $\mathbf{v} \in \Gamma\left(X \times A_{K}^{n}[d, e], G\right)$ by $\mathbf{v}=\sum_{J} \mathbf{v}_{J} t^{J}\left(\mathbf{v}_{J} \in V\right)$, we have $f(\mathbf{v})=\mathbf{v}_{\mathbf{0}}$, where $\mathbf{0}=(0, \ldots, 0)$.

Now we prove the claim 3 in the case $b>0$. Note that, for $\mathbf{v} \in \Gamma\left(X \times A_{K}^{n}[d, e], G\right)$, the series $\mathbf{v}=\sum_{J \in \mathbb{Z}^{n}} \mathbf{v}_{J} t^{J}$ converges in $G_{L}$, hence in $G$. Hence we obtain the equality $\mathbf{v}=\sum_{J \in \mathbb{Z}^{n}} f\left(t^{-J} \mathbf{v}\right) t^{J}$ in $G$. From this equality, we see that $\mathbf{v}$ is contained in $\pi_{X}^{*} H_{G}$ for any $\mathbf{v} \in \Gamma\left(X \times A_{K}^{n}[d, e], G\right)$. Since $\Gamma\left(X \times A_{K}^{n}[b, c], G\right)$ is dense in $\Gamma\left(X \times A_{K}^{n}[d, e], G\right)$, the canonical map $\pi_{X}^{*} H_{G} \longrightarrow G$ is surjective in the case $b>0$.

The claim 3 is true also in the case $b=0$ which we can prove in exactly the same way as [10, 3.4.3]. (The proof is more complicated, but the idea is similar. See [10, 3.4.3] for detail.)

By the claims 1 and 3 , we see that the canonical map $\pi_{X}^{*} H_{G} \longrightarrow G$ is in fact an isomorphism. Now we prove that $G$ is $\Sigma$-constant. If we enlarge $K$ in order that $A_{K}^{n}[b, c]$ contains a $K$-rational point $x$ and if $i_{x}: X \longrightarrow X \times A_{K}^{n}[b, c]$ denotes the section of $\pi_{X}$ induced by $x$, then we have $H_{G}=i_{x}^{*} \pi_{X}^{*} H_{G} \stackrel{\cong}{\longrightarrow} i_{x}^{*} G$. Hence $H_{G}$ is a locally free $\mathcal{O}_{X}$-module. (Note that this conclusion is true without enlarging $K$.) So $H_{G}=H_{X, \xi}^{0}\left(X \times A_{K}^{n}[b, c], G\right)$ is naturally regarded as an object in $\mathrm{LNM}_{X \times A_{K}^{n}[0,0], \Sigma}$ and we have $G=\mathcal{U}_{[b, c]}\left(H_{G}\right)$. Hence $G$ is $\Sigma$-constant.

Then, by induction on the rank of $E$, we can prove that $E / G$ is $\Sigma$-unipotent on $X \times A_{K}^{n}(b, c)$ when $b \neq 0$ and on $X \times A_{K}^{n}[0, c)$ when $b=0$. Hence so is $E$. Since $[b, c] \subseteq I$ is arbitrary, we see that $E$ is $\Sigma$-unipotent on $X \times A_{K}^{n}(I)$. So the proof is finished.

We have the following variant of Proposition 2.4, which is also important.

Corollary 2.5. Let $A$ be an affinoid algebra such that $X:=\operatorname{Spm} A$ is smooth and let $L$ be an affinoid algebra containing $A$ such that $\operatorname{Spm} L$ is smooth. Let I be a quasiopen interval of positive length in $[0,1)$, let $[b, c] \subseteq I$ be a closed aligned subinterval of positive length, let $\Sigma:=\prod_{i=1}^{n} \Sigma_{i}$ be a subset of $\mathbb{Z}_{p}^{n}$ which is (NID) and (NLD), and let $E$ be an object in $\operatorname{LNM}_{X \times A_{K}^{n}(I), \Sigma}$ such that the induced object $F \in \operatorname{LNM}_{\operatorname{Spm} L \times A_{K}^{n}(I), \Sigma}$ is $\Sigma$-unipotent and that $H_{X, \xi}^{0}\left(X \times A_{K}^{n}[b, c], E\right)$ is non-zero for some $\xi \in \Sigma$. Then $\left.E\right|_{X \times A_{K}^{n}[b, c]}$ contains a non-zero $\Sigma$-constant subobject $G \in \operatorname{LNM}_{X \times A_{K}^{n}[b, c], \Sigma}$.

Proof. By the proof of claims 1 and 2 in Proposition 2.4, we see that the $\mathcal{O}_{X \times A_{K}^{n}[b, c]^{-}}$ span of $H_{X, \xi}^{0}\left(X \times A_{K}^{n}[b, c], E\right)$ naturally defines a non-zero $\Sigma$-constant subobject $G \in \mathrm{LNM}_{X \times A_{K}^{n}[b, c], \Sigma}$. (Note that, since we do not have 'the log structure $x_{1}, \ldots, x_{r}$ ' on $X$ in the situation here, we have $X=X^{\prime}$ in the notation of the proof of Proposition 2.4. Note also that, although we do not assume that the spectral norm of $L$ is compatible with that of $A$, this does not cause any problem because we did not use 
this assumption in the proof of the claims 1 and 2 in Proposition 2.4.) So we are done.

Remark 2.6. Lemma 2.3 and Proposition 2.4 are slightly more generalized than the corresponding results in [10,3.4.1,3.4.3] in the sense that we treat the case (1). (In [10], only the case (2) is treated.) Also, Corollary 2.5 (which allows us to prove a kind of generization in the case where the spectrum norm on $L$ is not compatible with that on $A$ ) seems to be important. (See Remark 2.8 below.)

Next we prove the proposition called overconvergent generization, which is an analogue of (a slightly weaker form of) [10, 3.5.3]:

Proposition 2.7 (overconvergent generization). Let $P$ be a p-adic affine formal scheme topologically of finite type over $O_{K}$ and let $X \subseteq P_{k}$ be an open dense subscheme such that $P$ is formally smooth over $O_{K}$ on a neighborhood of $X$. Let $I \subseteq[0,1)$ be a quasi-open subinterval of positive length, let $V$ be a strict neighborhood of $] X{ }_{P}$ in $P_{K}$ and let $\Sigma=\prod_{i=1}^{n} \Sigma_{i}$ be a subset of $\mathbb{Z}_{p}^{n}$ which is (NID) and (NLD). Let $E$ be an object in $\mathrm{LNM}_{V \times A_{K}^{n}(I), \Sigma}$ whose restriction to $\mathrm{LNM}_{] X[P} \times A_{K}^{n}(I), \Sigma$ is $\Sigma$-unipotent. Then, for any closed aligned subinterval $[b, c] \subseteq I$ of positive length, there exists a strict neighborhood $V^{\prime}$ of $] X{ }_{P}$ in $P_{K}$ contained in $V$ such that the restriction of $E$ to $V^{\prime} \times A_{K}^{n}[b, c]$ is also $\Sigma$-unipotent.

Proof. We may assume that there exists $g \in \Gamma\left(P, \mathcal{O}_{P}\right)$ such that $X$ is defined as the locus $\{g \neq 0\}$ in $P_{k}$. For $\lambda \in[0,1) \cap \Gamma^{*}$, let us put $V_{\lambda}:=\left\{x \in P_{K}|| g(x) \mid \geq \lambda\right\}$. Then we may assume that $V=V_{\lambda_{0}}$ for some $\lambda_{0} \in[0,1) \cap \Gamma^{*}$ and that $V$ is smooth.

Take a sequence of aligned closed subintervals $[b, c] \subseteq\left[b^{\prime}, c^{\prime}\right] \subseteq[d, e]$ of $I$ with $d<b^{\prime}<b, c<c^{\prime}<e$ if $b>0$ and $d=b^{\prime}=0, c<c^{\prime}<e$ if $b=0$. Then, by the method of Lemma 2.3, we see that there exists an element $\mathbf{v} \in \Gamma\left(V \times A_{K}^{n}[d, e], E\right)$ such that $\left\{D_{l}(\mathbf{v})\right\}$ converges to a non-zero element in $H_{] X{ }_{P}, \xi}^{0}(] X\left[\times A_{K}^{n}\left[b^{\prime}, c^{\prime}\right], E\right)$ for some $\xi \in \Sigma$.

Let us take an affinoid open $W \subseteq V \times A_{K}^{n}[d, e]$ such that $E$ is free on $W$. We prove the following claim:

claim 1. On $W$, the sequence $\left\{D_{l}(\mathbf{v})-D_{l-1}(\mathbf{v})\right\}_{l}$ is $\rho$-null for some $\rho>0$.

First, we have

$$
\begin{aligned}
& \left|D_{l}(\mathbf{v})-D_{l-1}(\mathbf{v})\right| \\
= & \left|\left(\prod_{i=1}^{n} \prod_{k=1}^{n_{i}}\left(\frac{l-\left(t_{i} \frac{\partial}{\partial t_{i}}-\xi_{i k}\right)}{l-\left(\xi_{i 1}-\xi_{i k}\right)} \cdot \frac{l+\left(t_{i} \frac{\partial}{\partial t_{i}}-\xi_{i k}\right)}{l+\left(\xi_{i 1}-\xi_{i k}\right)}\right)^{m}-1\right) D_{l-1}(\mathbf{v})\right| \\
= & \mid\left(\prod _ { i = 1 } ^ { n } \prod _ { k = 1 } ^ { n _ { i } } \left(\frac{1}{l-\left(\xi_{i 1}-\xi_{i k}\right)} \cdot \frac{1}{l+\left(\xi_{i 1}-\xi_{i k}\right)} \cdot\right.\right. \\
& \left.\left.\quad\left(l-\left(t_{i} \frac{\partial}{\partial t_{i}}-\xi_{i k}\right)\right) \cdot\left(l+\left(t_{i} \frac{\partial}{\partial t_{i}}-\xi_{i k}\right)\right)\right)^{m}-1\right) D_{l-1}(\mathbf{v}) \mid
\end{aligned}
$$




$$
\leq C \cdot\left|\prod_{i=1}^{n} \prod_{k=1}^{n_{i}}\left(\frac{1}{l-\left(\xi_{i 1}-\xi_{i k}\right)} \cdot \frac{1}{l+\left(\xi_{i 1}-\xi_{i k}\right)}\right)\right|^{m} \cdot\left|D_{l-1}(\mathbf{v})\right| .
$$

(Here $C>1$ is a constant which is independent of $l$, which is written by using the action of the operators $t_{i} \frac{\partial}{\partial t_{i}}$ on the basis of $\left.E\right|_{W}$.) If we put $A_{l}:=$ $C \cdot\left|\prod_{i=1}^{n} \prod_{k=1}^{n_{i}}\left(\frac{1}{l-\left(\xi_{i 1}-\xi_{i k}\right)} \cdot \frac{1}{l+\left(\xi_{i 1}-\xi_{i k}\right)}\right)\right|^{m}$, we see from (2.5) the inequality $\left|D_{l}(\mathbf{v})-D_{l-1}(\mathbf{v})\right| \leq A_{l}\left|D_{l-1}(\mathbf{v})\right|$. Then we can prove the inequality

$$
\left|D_{l}(\mathbf{v})-D_{l-1}(\mathbf{v})\right| \leq\left(\prod_{j=i}^{l} A_{j}\right)\left|D_{i-1}(\mathbf{v})\right| \quad(1 \leq i \leq l)
$$

by descending induction: Indeed, (2.6) for $i=l$ is already shown, and if (2.6) is true for $i$, we have

$$
\begin{aligned}
\left|D_{l}(\mathbf{v})-D_{l-1}(\mathbf{v})\right| & \leq\left(\prod_{j=i}^{l} A_{j}\right)\left|D_{i-1}(\mathbf{v})\right| \\
& \leq\left(\prod_{j=i}^{l} A_{j}\right) \max \left(\left|D_{i-1}(\mathbf{v})-D_{i-2}(\mathbf{v})\right|,\left|D_{i-2}(\mathbf{v})\right|\right) \\
& \leq\left(\prod_{j=i}^{l} A_{j}\right) \max \left(A_{i-1}\left|D_{i-2}(\mathbf{v})\right|,\left|D_{i-2}(\mathbf{v})\right|\right)=\left(\prod_{j=i-1}^{l} A_{j}\right)\left|D_{i-2}(\mathbf{v})\right| .
\end{aligned}
$$

In particular, we have the inequality $\left|D_{l}(\mathbf{v})-D_{l-1}(\mathbf{v})\right| \leq\left(\prod_{j=1}^{l} A_{j}\right)|\mathbf{v}|$. Let us calculate $\prod_{j=1}^{l} A_{j}$. By definition, we have

$$
\prod_{j=1}^{l} A_{j}=C^{l}\left|\prod_{i=1}^{n} \prod_{k=1}^{n_{i}}\left\{\left(\prod_{j=1}^{l} \frac{1}{j-\left(\xi_{i 1}-\xi_{i k}\right)}\right)^{m}\left(\prod_{j=1}^{l} \frac{1}{j+\left(\xi_{i 1}-\xi_{i k}\right)}\right)^{m}\right\}\right| .
$$

Since $\pm\left(\xi_{i 1}-\xi_{i k}\right)$ are $p$-adically non-Liouville, we see from [9, VI Lemma 1.2] that, for any $\zeta>p^{1 /(p-1)}$, we have the inequalities

$$
\left|\prod_{j=1}^{l} \frac{1}{j \pm\left(\xi_{i 1}-\xi_{i k}\right)}\right| \leq(\text { const }) \cdot \zeta^{l} .
$$

Hence we have $\prod_{j=1}^{l} A_{j} \leq$ (const) $\cdot\left(C \zeta^{2 m}\right)^{l}$. Therefore, if we put $\rho:=\left(C \zeta^{2 m}\right)^{-2}$, we see that the sequence $\left\{D_{l}(\mathbf{v})-D_{l-1}(\mathbf{v})\right\}_{l}$ is $\rho$-null. So we have proved the claim 1 .

When $W \cap(] X\left[\times A_{K}^{n}[d, e]\right)$ is not empty, we see from claim 2 in Lemma 2.3 that the sequence $\left\{D_{l}(\mathbf{v})-D_{l-1}(\mathbf{v})\right\}_{l}$ is $\eta$-null for some $\eta>1$ on $] X\left[\times A_{K}^{n}\left[b^{\prime}, c^{\prime}\right]\right.$. On the other hand, by claim 1 , it is $\rho$-null for some $\rho>0$ on $W \cap\left(V \times A_{K}^{n}\left[b^{\prime}, c^{\prime}\right]\right)$. 
Hence, by [10, 3.5.2], we see that it is 1-null on $W \cap\left(V_{\lambda} \times A_{K}^{n}\left[b^{\prime}, c^{\prime}\right]\right)$ for some $\lambda \in\left(\lambda_{0}, 1\right) \cap \Gamma^{*}$. On the other hand, when $W \cap(] X\left[\times A_{K}^{n}[d, e]\right)$ is empty, then we see that $W \cap\left(V_{\lambda} \times A_{K}^{n}\left[b^{\prime}, c^{\prime}\right]\right)$ is also empty for some $\lambda$. (See the proof of [10, 3.5.3].)

By taking a covering of $V \times A_{K}^{n}[d, e]$ by affinoids $W$ on which $E$ is free and argueing as above, we see that there exists some $\lambda \in\left(\lambda_{0}, 1\right) \cap \Gamma^{*}$ such that the limit $\lim _{l} D_{l}(\mathbf{v})$ exists on $V_{\lambda} \times A_{K}^{n}\left[b^{\prime}, c^{\prime}\right]$, which is non-zero for some $\mathbf{v}$. Hence we have $H_{V_{\lambda}, \xi}^{0}\left(V_{\lambda} \times A_{K}^{n}\left[b^{\prime}, c^{\prime}\right], E\right) \neq 0$. Then, by Corollary 2.5 (with $A=\Gamma\left(V_{\lambda}, \mathcal{O}\right), L=\Gamma(] X[$, $\mathcal{O})$ ), we see that $E$ contains a $\Sigma$-constant subobject $G$ on $V_{\lambda} \times A_{K}^{n}\left[b^{\prime}, c^{\prime}\right]$. Then, $E / G$ satisfies the assumption of the proposition required for $E$ if we replace $I$ by $\left(b^{\prime}, c^{\prime}\right)$. Hence, by induction on the rank of $E$, we can assume that $E / G$ is $\Sigma$-unipotent on $V_{\lambda} \times A_{K}^{n}[b, c]$ for some $\lambda$. Hence $E$ is $\Sigma$-unipotent on $V_{\lambda} \times A_{K}^{n}[b, c]$ for some $\lambda$ and so we are done.

Remark 2.8. Proposition 2.7 is slightly weaker than the corresponding result in [10, 3.5.3], because we do not put 'the log structure' on $P_{K}$ here. However, it seems to us that there is an error in the proof of [10, 3.5.3]: At the final step of the proof there, it is written that 'the role of $L$ in the proof of Proposition 3.4.3 is played by a complete field containing $\mathcal{O}(] X[)$ whose norm is compatible with the norm on $\mathcal{O}\left(V_{\lambda}\right)^{\prime}$. But such a field $L$ does not exist in general because the reduction of the affinoid algebra $\mathcal{O}\left(V_{\lambda}\right)$ is not an integral domain in general. (See also the statement of [10, 3.4.1].)

Note that, in the proof of [10, 3.4.3], he uses the fact that the norm of $L$ is compatible with that of $\mathcal{O}(V)$ : It is used in the proof of the assertion corresponding to the claim 3 in Proposition 2.4 here. On the other hand, we saw that, in the proof of Corollary 2.5, we do not need the compatibility of the norms of $L$ and that of $A$. Moreover, in the proof of Proposition 2.7, we used only Corollary 2.5, not Proposition 2.4. This is the reason that our Proposition 2.7 is slightly weaker. However, as we will see in the next section, this does not cause any problem in proving our main result.

We would like to note that there is another way to fix the above-mentioned error, which is explained in [11, Appendix A].

Now we proceed to prove the third key proposition. To do this, we introduce the notion of log-convergence of a log- $\nabla$-module.

Definition 2.9. Let $X$ be a smooth affinoid rigid space endowed with $x_{1}, \ldots, x_{r} \in$ $\Gamma\left(X, \mathcal{O}_{X}\right)$ whose zero loci are smooth and meet transversally, let $a \in(0,1] \cap \Gamma^{*}$ and let $E$ be a log- $\nabla$-module on $X \times A_{K}^{n}[0, a)$ with respect to $x_{1}, \ldots, x_{r}, t_{1}, \ldots, t_{n}$. Then $E$ is called log-convergent if, for any $a^{\prime} \in(0, a) \cap \Gamma^{*}, \eta \in(0,1)$ and $\mathbf{v} \in \Gamma\left(X \times A_{K}^{n}\left[0, a^{\prime}\right], E\right)$, the multisequence

$$
\left\{\frac{1}{i_{1} ! \cdots i_{n} !}\left(\prod_{j=1}^{n} \prod_{l=0}^{i_{j}-1}\left(t_{j} \frac{\partial}{\partial t_{j}}-l\right)\right)(\mathbf{v})\right\}_{i_{1}, \ldots, i_{n}}
$$

is $\eta$-null on $X \times A_{K}^{n}\left[0, a^{\prime}\right]$. 
For a multi-index $I:=\left(i_{1}, \ldots, i_{n}\right) \in \mathbb{N}^{n}$ and a multi-variable $x:=\left(x_{1}, \ldots, x_{n}\right)$, we put $P_{I}(x):=\frac{1}{i_{1} ! \cdots i_{n} !}\left(\prod_{j=1}^{n} \prod_{l=0}^{i_{j}-1}\left(x_{j}-l\right)\right)$. Then the multi-sequence in Definition 2.9 can be written as $\left\{P_{I}\left(t \frac{\partial}{\partial t}\right)(\mathbf{v})\right\}_{I}$ with this notation. The operator $P_{I}\left(t \frac{\partial}{\partial t}\right)$ is the same as the operator $t^{I}\left(\frac{\partial}{\partial t}\right)^{I}$ in the notation of [2, 3.7.2].

Remark 2.10. The definition of log-convergence given above is closely related to the notion of 'overconvergence' defined in [2, 5.1] in the case $a=1$ : Indeed, they are equivalent if $r=0$ and if $E$ has the form $\pi^{*} F$ for some coherent sheaf $F$ on $X$, where $\pi$ denotes the projection $X \times A_{K}^{n}[0,1) \longrightarrow X$.

It is known $([2,3.7 .3])$ that, for $f \in \Gamma\left(X \times A_{K}^{n}\left[0, a^{\prime}\right], \mathcal{O}\right)$ and $\mathbf{v} \in \Gamma(X \times$ $\left.A_{K}^{n}\left[0, a^{\prime}\right], E\right)$, we have

$$
P_{I}\left(t \frac{\partial}{\partial t}\right)(f \mathbf{v})=\sum_{0 \leq I^{\prime} \leq I} P_{I^{\prime}}\left(t \frac{\partial}{\partial t}\right)(f) \cdot P_{I-I^{\prime}}\left(t \frac{\partial}{\partial t}\right)(\mathbf{v}) .
$$

Hence we have

$$
\begin{aligned}
\left|P_{I}\left(t \frac{\partial}{\partial t}\right)(f \mathbf{v})\right| & \leq \sup _{0 \leq I^{\prime} \leq I}\left|P_{I^{\prime}}\left(t \frac{\partial}{\partial t}\right)(f)\right|\left|P_{I-I^{\prime}}\left(t \frac{\partial}{\partial t}\right)(\mathbf{v})\right| \\
& \leq|f| \sup _{0 \leq I^{\prime} \leq I}\left|P_{I-I^{\prime}}\left(t \frac{\partial}{\partial t}\right)(\mathbf{v})\right|,
\end{aligned}
$$

where the second inequality follows from [2, 5.2]. Hence, for $\mathbf{v}=\sum_{j} f_{j} \mathbf{v}_{j}$, we have

$$
\left|P_{I}\left(t \frac{\partial}{\partial t}\right)(\mathbf{v})\right| \leq \sup _{j}\left(\left|f_{j}\right| \sup _{0 \leq I^{\prime} \leq I}\left|P_{I-I^{\prime}}\left(t \frac{\partial}{\partial t}\right)\left(\mathbf{v}_{j}\right)\right|\right) \text {. }
$$

So we have

$$
\left|P_{I}\left(t \frac{\partial}{\partial t}\right)(\mathbf{v})\right| \eta^{|I|} \leq \sup _{j}\left(\left|f_{j}\right| \sup _{0 \leq I^{\prime} \leq I}\left|P_{I-I^{\prime}}\left(t \frac{\partial}{\partial t}\right)\left(\mathbf{v}_{j}\right)\right| \eta^{\left|I-I^{\prime}\right| / 2}\right) \cdot \eta^{|I| / 2}
$$

for $\eta<1$. From (2.8), we see that, to check the $\eta$-nullity of the multisequence (2.7) for all $\mathbf{v} \in \Gamma\left(X \times A_{K}^{n}\left[0, a^{\prime}\right], E\right)$, it suffices to check it only for a set of generators of $\Gamma\left(X \times A_{K}^{n}\left[0, a^{\prime}\right], E\right)$. Also, (2.8) shows that $E$ is log-convergent if and only if the multi-sequence $\left|P_{I}\left(t \frac{\partial}{\partial t}\right)\right| \eta^{|I|}$ converges to zero for any $a^{\prime} \in(0, a) \cap \Gamma^{*}$, where $\left|P_{I}\left(t \frac{\partial}{\partial t}\right)\right|$ denotes the operator norm of $P_{I}\left(t \frac{\partial}{\partial t}\right)$ on $\Gamma\left(X \times A_{K}^{n}\left[0, a^{\prime}\right], E\right)$.

Note that the trivial $\log -\nabla$-module $(\mathcal{O}, d)$ on $X \times A_{K}^{n}[0,1)$ is log-convergent, since we have $P_{I}\left(t \frac{\partial}{\partial t}\right)(1)=0$ for $I$ with $|I|>0$. Also, we remark here that, if there exists a exact sequence

$$
0 \longrightarrow E^{\prime} \longrightarrow E \longrightarrow E^{\prime \prime} \longrightarrow 0
$$

of $\log$ - $\nabla$-modules on $X \times A_{K}^{n}[0, a)$ with respect to $x_{1}, \ldots, x_{r}, t_{1}, \ldots, t_{n}$ with $E^{\prime}, E^{\prime \prime} \log$ convergent, then $E$ is also log-convergent: Indeed, if we fix an isomorphism $E \cong E^{\prime} \oplus$ 
$E^{\prime \prime}$ as $\mathcal{O}_{X \times A_{K}^{n}\left[0, a^{\prime}\right]}$-modules and if we write $t_{j} \frac{\partial}{\partial t_{j}}$ on $E$ as $\left(\begin{array}{cc}\left(t_{j} \frac{\partial}{\partial t_{j}}\right)^{\prime} & \Gamma_{j} \\ 0 & \left(t_{j} \frac{\partial}{\partial t_{j}}\right)^{\prime \prime}\end{array}\right)$

(where $\left(t_{j} \frac{\partial}{\partial t_{j}}\right)^{\prime},\left(t_{j} \frac{\partial}{\partial t_{j}}\right)^{\prime \prime}$ are the operator $t_{j} \frac{\partial}{\partial t_{j}}$ for $E^{\prime}, E^{\prime \prime}$, respectively), we obtain, by a tedious calculation, an inequality

$$
\left|P_{I}\left(t \frac{\partial}{\partial t}\right)\right| \leq\left(\sup _{0 \leq \alpha \leq|I|}|1 / \alpha|\right) \cdot \sup _{I^{\prime}+I^{\prime \prime}<I}\left(\left|P_{I^{\prime}}\left(\left(t \frac{\partial}{\partial t}\right)^{\prime}\right)\right|\left|P_{I^{\prime \prime}}\left(\left(t \frac{\partial}{\partial t}\right)^{\prime \prime}\right)\right|\right) \cdot \sup _{j}\left|\Gamma_{j}\right| \text {. }
$$

Hence we have, for $\eta<1$, the inequality

$$
\begin{aligned}
& \left|P_{I}\left(t \frac{\partial}{\partial t}\right)\right| \eta^{|I|} \\
& \leq \text { (const) } \cdot \log |I| \cdot \sup _{I^{\prime}+I^{\prime \prime}<I}\left(\left|P_{I^{\prime}}\left(\left(t \frac{\partial}{\partial t}\right)^{\prime}\right)\right|\left|P_{I^{\prime \prime}}\left(\left(t \frac{\partial}{\partial t}\right)^{\prime \prime}\right)\right| \eta^{\left(\left|I^{\prime}\right|+\left|I^{\prime \prime}\right|\right) / 2}\right) \cdot \eta^{|I| / 2} .
\end{aligned}
$$

From this we see that the $\log$ convergence of $E^{\prime}$ and $E^{\prime \prime}$ implies that of $E$.

Now we prove some relations between log-convergence and $\Sigma$-unipotence. The following (certainly well-known) lemma, which can be regarded as a variant of [10, 3.6.1], shows that $\Sigma$-unipotent $\log -\nabla$-module is log-convergent under certain condition:

Lemma 2.11. Let $X$ be a smooth affinoid rigid space endowed with $x_{1}, \ldots, x_{r} \in$ $\Gamma\left(X, \mathcal{O}_{X}\right)$ whose zero loci are smooth and meet transversally. Let $\Sigma=\Sigma^{\prime} \times \prod_{i=1}^{n} \Sigma_{i}$ be a subset of $\bar{K}^{r} \times \mathbb{Z}_{p}^{n}$ and let $E$ be a $\Sigma$-unipotent log- $\nabla$-module on $X \times A_{K}^{n}[0,1)$ with respect to $x_{1}, \ldots, x_{r}, t_{1}, \ldots, t_{n}$. Then $E$ is log-convergent.

Proof. Since the log-convergence is closed under extension, we may assume that $E$ is $\Sigma$-constant. Hence we may assume that $E$ has the form $\pi_{1}^{*} F \otimes \pi_{2}^{*} M_{\xi}$ for some $F \in \operatorname{LNM}_{X, \Sigma^{\prime}}$ and $\xi=\left(\xi_{1}, \ldots, \xi_{n}\right) \in \mathbb{Z}_{p}^{n}$, where $\pi_{1}: X \times A_{K}^{n}[0,1) \longrightarrow X$, $\pi_{2}: X \times A_{K}^{n}[0,1) \longrightarrow A_{K}^{n}[0,1)$ are projections. If we take a generator $\mathbf{v}_{1}, \ldots, \mathbf{v}_{d}$ of $\Gamma(X, F)$, it forms a generator of $\Gamma\left(X \times A_{K}^{n}[0, a], E\right)$ for any $a \in(0,1) \cap \Gamma^{*}$ and we have the inequality

$$
\begin{aligned}
\left|P_{I}\left(t \frac{\partial}{\partial t}\right)\left(\mathbf{v}_{s}\right)\right| & =\left|\frac{1}{i_{1} ! \cdots i_{n} !} \prod_{j=1}^{n} \prod_{l=0}^{i_{j}-1}\left(\xi_{j}-l\right)\left(\mathbf{v}_{s}\right)\right| \\
& =\left|\prod_{j=1}^{n} \frac{\xi_{j}\left(\xi_{j}-1\right) \cdots\left(\xi_{j}-i_{j}+1\right)}{i_{j} !}\left(\mathbf{v}_{s}\right)\right| \leq\left|\mathbf{v}_{s}\right| .
\end{aligned}
$$

From this we see that $E$ is log-convergent. 
The following proposition, which is the third key proposition in this section, shows that under some condition, log-convergence implies $\Sigma$-unipotence. This is a variant of the transfer theorems in [5], [1, [2, 6.5.2] and [10, 3.6.2].

Proposition 2.12. Let $A$ be an integral affinoid algebra such that $X:=\operatorname{Spm} A$ is smooth and endowed with $x_{1}, \ldots, x_{r} \in A$ whose zero loci are smooth and meet transversally. Assume moreover that there exists $A \subseteq L$ satisfying one of the conditions (1), (2) in Proposition 2.4. Let $\Sigma=\Sigma^{\prime} \times \prod_{i=1}^{n} \Sigma_{i}$ be a subset of $\bar{K}^{r} \times \mathbb{Z}_{p}^{n}$ which is (NID) and (NLD). Then, if $E$ is an object in $\operatorname{LNM}_{X \times A_{K}^{n}[0,1), \Sigma}$ which is log-convergent, it is $\Sigma$-unipotent.

Proof. Let $F$ be the object in $\operatorname{LNM}_{A_{L}^{n}[0,1), \Sigma}$ induced by $E$. Then $F$ is also logconvergent. By Proposition 2.4, it suffices to prove that $F$ is $\Sigma$-unipotent, and it is enough to prove that $F$ is $\Sigma$-unipotent on $A_{L}^{n}[0, a)$ for any $a \in(0,1) \cap \Gamma^{*}$. By induction on the rank of $F$, we see that it suffices to prove the following claim: Let $a^{\prime}<a$ be elements in $(0,1) \cap \Gamma^{*}$. Then, if $F$ is an object in $\operatorname{LNM}_{A_{L}^{n}[0, a), \Sigma}$ which is log-convergent, $F$ contains a $\Sigma$-constant subobject on $A_{L}^{n}\left[0, a^{\prime}\right)$. We will prove this claim.

Let $F_{0}$ be the pull-back of $F$ by the map $\operatorname{Spm} L \hookrightarrow A_{L}^{n}[0,1)$ of 'inclusion into the origin'. Then $F_{0}$ admits commuting endomorphisms $N_{i}(1 \leq i \leq n)$ coming from the residues along $t_{i}=0$. Let $\xi_{i k} \in \Sigma_{i}\left(1 \leq k \leq n_{i}\right)$ be the eigenvalues of $N_{i}$ and let $P_{i}(x)=\prod_{k=1}^{n_{i}}\left(x-\xi_{i k}\right)^{m_{i k}}$ be the minimal polynomial of $N_{i}$. Let us put $m=\max _{i, k}\left(m_{i k}\right)$. For $\mathbf{k}:=\left(k_{1}, \ldots, k_{n}\right)\left(1 \leq k_{i} \leq n_{i}\right)$, let us define $F_{0, \mathbf{k}}^{\prime}:=$ $\left\{x \in F_{0} \mid \forall i,\left(N_{i}-\xi_{i k_{i}}\right)(x)=0\right\}$. We may assume that $F_{0, \mathbf{1}}^{\prime} \neq\{0\}$, where we put $\mathbf{1}:=(1, \ldots, 1)$. Let us take polynomials $Q_{i}(x)(1 \leq i \leq n)$ dividing $P_{i}(x)$ such that the map $\prod_{i=1}^{n} Q_{i}\left(N_{i}\right): F_{0} \longrightarrow F_{0}$ is a non-zero map whose image is contained in $F_{0,1}^{\prime}$.

Now let us define a polynomial $D_{l}(x)$ with variable $x:=\left(x_{1}, \ldots, x_{n}\right)$ by

$$
D_{l}(x):=\prod_{i=1}^{n} D_{l, i}\left(x_{i}\right):=\prod_{i=1}^{n}\left\{Q_{i}\left(x_{i}\right) \prod_{k=1}^{n_{i}} \prod_{j=1}^{l}\left(\frac{j-\left(x_{i}-\xi_{i k}\right)}{j-\left(\xi_{i 1}-\xi_{i k}\right)}\right)^{m}\right\} .
$$

On the other hand, let us put $P_{l}(y):=\prod_{j=1}^{l} \frac{(j-1)-y}{j}$. Then we have

$$
\begin{aligned}
D_{l, i}(x) & =Q_{i}(x) \prod_{k=1}^{n_{i}}\left\{\left(\prod_{j=1}^{l} \frac{j}{j-\left(\xi_{i 1}-\xi_{i k}\right)}\right)^{m} \cdot P_{l}\left(x-\left(\xi_{i k}+1\right)\right)^{m}\right\} \\
& =Q_{i}(x) \prod_{k=1}^{n_{i}}\left\{\left(\prod_{j=1}^{l} \frac{j}{j-\left(\xi_{i 1}-\xi_{i k}\right)}\right)^{m} \cdot\left(\sum_{q=0}^{l} P_{l-q}\left(-\left(\xi_{i k}+1\right)\right) P_{q}(x)\right)^{m}\right\} .
\end{aligned}
$$

If we put $c_{i l}:=\prod_{k=1}^{n_{i}}\left(\prod_{j=1}^{l} \frac{j}{j-\left(\xi_{i 1}-\xi_{i k}\right)}\right)^{m}$, we see that $D_{l, i}(x)$ has the form

$$
c_{i l} Q_{i}(x) \cdot\left(\mathbb{Z}_{p} \text {-linear combination of } P_{q_{1}}(x) \cdots P_{q_{m}}(x)\left(0 \leq q_{i} \leq l\right)\right) .
$$


Let us fix some $a^{\prime \prime} \in\left(a^{\prime}, a\right) \cap \Gamma^{*}$. Then, for any $\eta \in(0,1)$, we have the following inequality concerning the norms of operators on $A_{L}^{n}\left[0, a^{\prime \prime}\right]$ :

$$
\begin{aligned}
& \left|D_{l}\left(t \frac{\partial}{\partial t}\right)\right| \eta^{l} \\
\leq & \left|\prod_{i=1}^{n} Q_{i}\left(t_{i} \frac{\partial}{\partial t_{i}}\right)\right| \cdot\left|\prod_{i=1}^{n} c_{i l}\right| \cdot \prod_{i=1}^{n} \sup _{0 \leq q_{1}, \ldots, q_{m} \leq l}\left(\left|P_{q_{1}}\left(t_{i} \frac{\partial}{\partial t_{i}}\right)\right| \ldots\left|P_{q_{m}}\left(t_{i} \frac{\partial}{\partial t_{i}}\right)\right|\right) \eta^{l} \\
\leq & (\text { const })\left|\prod_{i=1}^{n} c_{i l}\right| \eta^{l / 2} \prod_{i=1}^{n} \sup _{0 \leq q_{1}, \ldots, q_{m} \leq l}\left(\left|P_{q_{1}}\left(t_{i} \frac{\partial}{\partial t_{i}}\right)\right| \eta^{q_{1} / 2 n m} \ldots\left|P_{q_{m}}\left(t_{i} \frac{\partial}{\partial t_{i}}\right)\right| \eta^{q_{m} / 2 n m}\right) .
\end{aligned}
$$

On the right hand side on (2.9),$\left|\prod_{i=1}^{n} c_{i l}\right| \eta^{l / 2}$ converges to zero when $l \rightarrow \infty$ and the term $\sup _{0 \leq q_{1}, \ldots, q_{m} \leq l}\left(\left|P_{q_{1}}\left(t_{i} \frac{\partial}{\partial t_{i}}\right)\right| \eta^{q_{1} / 2 n m} \ldots\left|P_{q_{m}}\left(t_{i} \frac{\partial}{\partial t_{i}}\right)\right| \eta^{q_{m} / 2 n m}\right)$ is known to be bounded above, by the log-convergence of $F$. Hence we have shown that $\left|D_{l}\left(t \frac{\partial}{\partial t}\right)\right| \eta^{l}$ converges to zero as $l \rightarrow \infty$.

If $b \in\left(0, a^{\prime \prime}\right] \cap \Gamma^{*}$ is small enough, $F$ is $\Sigma$-unipotent on $A_{L}^{n}[0, b]$. (In fact, if $L$ is as in (2) in Proposition 2.4, it follows from Lemma 1.10, If $L$ is as in (1) in Proposition 2.4. $\operatorname{Spm} L$ admits a finite admissible open affinoid covering $\operatorname{Spm} L=\bigcup_{i} \operatorname{Spm} L_{i}$ such that $\left.F\right|_{\mathrm{Spm} L_{i} \times\{0\}}$ is free. Then, by Lemma 1.10 and Corollaries 1.15, 1.16, $\left.F\right|_{A_{L_{i}}^{n}[0, b]}$ has the form $\mathcal{U}_{[0, b]}\left(F_{i}^{\prime}\right)$ for a unique $F_{i}^{\prime} \in \operatorname{LNM}_{A_{L_{i}}^{n}[0,0], \Sigma}$. Using Corollary 1.16, we can glue $F_{i}^{\prime}$ 's to define an object $F^{\prime}$ in $\operatorname{LNM}_{A_{L}^{n}[0,0], \Sigma}$ with $\mathcal{U}_{[0, b]}\left(F^{\prime}\right)=F$.) Then, the direct calculation similar to the proof of Lemma 2.3 shows that the image of $D_{l+1}\left(t \frac{\partial}{\partial t}\right)-D_{l}\left(t \frac{\partial}{\partial t}\right)$ on $\Gamma\left(A_{L}^{n}[0, b], F\right)$ is contained in $t_{1}^{l} \cdots t_{n}^{l} \Gamma\left(A_{L}^{n}[0, b], F\right)$. Hence the image of $D_{l+1}\left(t \frac{\partial}{\partial t}\right)-D_{l}\left(t \frac{\partial}{\partial t}\right)$ on $\Gamma\left(A_{L}^{n}\left[0, a^{\prime \prime}\right], F\right)$ is contained in

$$
\Gamma\left(A_{L}^{n}\left[0, a^{\prime \prime}\right], F\right) \cap t_{1}^{l} \cdots t_{n}^{l} \Gamma\left(A_{L}^{n}[0, b], F\right)=t_{1}^{l} \cdots t_{n}^{l} \Gamma\left(A_{L}^{n}\left[0, a^{\prime \prime}\right], F\right) .
$$

Hence the operator $\frac{1}{t_{1}^{l} \cdots t_{n}^{l}}\left(D_{l+1}\left(t \frac{\partial}{\partial t}\right)-D_{l}\left(t \frac{\partial}{\partial t}\right)\right)$ is well-defined as an operator on $\Gamma\left(A_{L}^{n}\left[0, a^{\prime \prime}\right], F\right)$.

Now take any $c \in(0,1) \cap \Gamma^{*}$ and $\eta \in(0,1)$ with $\eta<c<a^{\prime \prime}$. Then, since $\left|D_{l}\left(t \frac{\partial}{\partial t}\right)\right|(\eta / c)^{n l}$ converges to zero as $l \rightarrow \infty,\left|D_{l+1}\left(t \frac{\partial}{\partial t}\right)-D_{l}\left(t \frac{\partial}{\partial t}\right)\right|(\eta / c)^{n l}$ converges to zero as $l \rightarrow \infty$ on $A_{L}^{n}\left[0, a^{\prime \prime}\right]$. Hence, for any $\mathbf{v} \in \Gamma\left(A_{L}^{n}\left[0, a^{\prime \prime}\right], F\right)$,

$$
\left|\frac{1}{t_{1}^{l} \cdots t_{n}^{l}}\left(D_{l+1}\left(t \frac{\partial}{\partial t}\right)-D_{l}\left(t \frac{\partial}{\partial t}\right)\right)(\mathbf{v})\right| \eta^{n l}
$$

converges to zero on $A_{L}^{n}\left[c, a^{\prime \prime}\right]$ as $l \rightarrow \infty$. (Here we use [10, 3.1.8].) Then, by [10, 3.1.6], it converges to zero on $A_{L}^{n}\left[0, a^{\prime \prime}\right]$ as $l \rightarrow \infty$. Since this is true for any $\eta<a^{\prime \prime}$ 
as above, we see that, for any $\mathbf{v} \in \Gamma\left(A_{L}^{n}\left[0, a^{\prime \prime}\right], F\right),\left|\left(D_{l+1}\left(t \frac{\partial}{\partial t}\right)-D_{l}\left(t \frac{\partial}{\partial t}\right)\right)(\mathbf{v})\right|$ converges to zero on $A_{L}^{n}\left[0, a^{\prime}\right]$ as $l \rightarrow \infty$. Hence the limit $f(\mathbf{v})=\lim _{l} D_{l}(\mathbf{v})$ exists on $A_{L}^{n}\left[0, a^{\prime}\right]$. We see, by the similar argument to the proof of Lemma 2.3 , that $f(\mathbf{v})$ defines an element in $H_{L, \xi}^{0}\left(A_{L}^{n}\left[0, a^{\prime}\right], F\right)$ (where $\left.\xi:=\left(\xi_{11}, \cdots, \xi_{n 1}\right)\right)$ and it is non-zero for some $\mathbf{v} \in \Gamma\left(A_{L}^{n}\left[0, a^{\prime \prime}\right], F\right)$.

In the case where $L$ is as in (2) in Proposition 2.4, we can define a morphism $M_{\xi} \longrightarrow F$ in $\operatorname{LNM}_{A_{L}^{n}\left[0, a^{\prime}\right], \Sigma}$ which sends 1 to $f(\mathbf{v})$ and it is injective because it is non-zero and the rank of $M_{\xi}$ is one. So we have shown that $F$ has a non-zero $\Sigma$ constant subobject on $A_{L}^{n}\left[0, a^{\prime}\right]$. So the proof of the claim in the first paragraph is done and hence the proof of the proposition is finished in this case.

In the case where $L$ is as in (1) in Proposition 2.4, we need to work more. Let us put $H:=H_{L, \xi}^{0}\left(A_{L}^{n}\left[0, a^{\prime}\right], F\right)$. Then, to finish the proof of the the claim in the first paragraph (and the proof of the proposition), it suffices to prove the following claims:

claim 1. $\quad H$ is a finitely generated $L$-module and the canonical map $\pi_{a^{\prime}}^{*} H \longrightarrow F$ (where $\pi_{a^{\prime}}: A_{L}^{n}\left[0, a^{\prime}\right] \longrightarrow \operatorname{Spm} L$ is the projection) is injective.

claim 2. $\pi_{a^{\prime}}^{*} H$ has a natural structure of a $\Sigma$-constant $\log$ - $\nabla$-module on $A_{L}^{n}\left[0, a^{\prime}\right]$ which is a subobject of $F$.

We give a proof of these claims, following the proof of the corresponding claims in Proposition [2.4. Remember that we have $b \in\left(0, a^{\prime \prime}\right] \cap \Gamma^{*}$ such that $\left.F\right|_{A_{L}^{n}[0, b]}$ has the form $\mathcal{U}_{[0, b]}\left(F^{\prime}\right)$ for some $F^{\prime} \in \operatorname{LNM}_{A_{L}^{n}[0,0], \Sigma}$ (and so it is $\Sigma$-unipotent). Let $\pi_{b}: A_{L}^{n}[0, b] \longrightarrow \mathrm{Spm} L$ be the projection and for a finitely generated $L$-submodule $M$ of $H$, consider the following diagram:

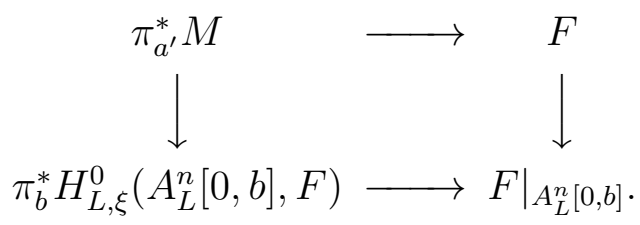

Then the vertical arrows are injective and we can prove the injectivity of the lower horizontal arrow by using the expression $\left.F\right|_{A_{L}^{n}[0, b]}=\mathcal{U}_{[0, b]}\left(F^{\prime}\right)$, as in the proof of Proposition 2.4. So the map $\pi_{a^{\prime}}^{*} M \longrightarrow F$ is injective and by using the Noetherianness of $F$, we can prove that $H$ is finitely generated and that the map $\pi_{a^{\prime}}^{*} H \longrightarrow F$ is injective in the same way as the proof of claim 1 in Proposition 2.4. So we have proved the claim 1.

Then, since $H$ is stable by the action of continuous derivations of $\operatorname{Spm} L$ over

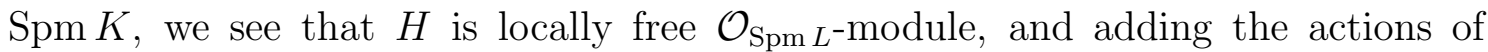
$t_{i}\left(\partial / \partial t_{i}\right)\left(=\right.$ multiplication by $\left.\xi_{i 1}\right)$, we see that $H$ defines a $\Sigma$-constant object in $\operatorname{LNM}_{A_{L}^{n}[0,0], \Sigma}$. Hence $\pi_{a^{\prime}}^{*} H=\mathcal{U}_{\left[0, a^{\prime}\right]}(H)$ has a natural structure of a $\Sigma$-constant log$\nabla$-module on $A_{L}^{n}\left[0, a^{\prime}\right]$ which is a subobject of $F$, as desired. So the proof of the claim 2 is also finished and we are done. 


\section{Monodromy of isocrystals and logarithmic ex- tension}

Let us assume given an open immersion $X \hookrightarrow \bar{X}$ of smooth $k$-varieties with $Z:=$ $\bar{X}-X=\bigcup_{i=1}^{r} Z_{i}$ a simple normal crossing divisor and let us denote the log structure on $\bar{X}$ induced by $Z$ by $M$. In this section, we introduce the notion of 'having $\Sigma$ unipotent monodromy' for an overconvergent isocrystal on $(X, \bar{X}) / K$ and prove that, under the assumption that $\Sigma=\prod_{i=1}^{r} \Sigma_{i} \subseteq \mathbb{Z}_{p}^{r}$ is (NID) and (NLD), any overconvergent isocrystal on $(X, \bar{X}) / K$ having $\Sigma$-unipotent monodromy uniquely extends to an isocrystal on log convergent site $\left((\bar{X}, M) / O_{K}\right)_{\text {conv }}$ with exponents in $\Sigma$.

Also, we can give the following expression of the above result. For an overconvergent isocrystal on $(X, \bar{X}) / K$, we define the notion of the Robba condition (see Definition 3.13 for detail). Then, by combining the result stated in the previous paragraph and a little argument, we prove the following: If we fix a section $\tau:\left(\mathbb{Z}_{p} / \mathbb{Z}\right)^{r} \longrightarrow \mathbb{Z}_{p}^{r}$ of the canonical projection, any overconvergent isocrystal satisfying the Robba condition and the condition (NLD) (which stands for "nonLiouville difference' of exponents and will be defined below) uniquely extends to an isocrystal on log convergent site $\left((\bar{X}, M) / O_{K}\right)_{\text {conv }}$ with exponents in $\tau\left(\bar{\Sigma}_{\mathcal{E}}\right)$, where $\bar{\Sigma}_{\mathcal{E}} \subseteq\left(\mathbb{Z}_{p} / \mathbb{Z}\right)^{r}$ denotes the set of Christol-Mebkhout exponents of $\mathcal{E}$ which will also be defined below. Since the Robba condition is regarded as a $p$-adic analogue of the regular singularity of integrable connections, this result can be regarded as a $p$-adic analogue of the canonical extension of regular singular integrable connections in $[8$, II 5.4].

Throughout this section, we assume that $K$ is discretely valued. First we recall terminologies on frames in [10].

Definition 3.1 ([10, 2.2.4]). A frame (or affine frame) is a tuple $(X, \bar{X}, P, i, j)$, where $X, \bar{X}$ are $k$-varieties, $P$ is a p-adic affine formal scheme topologically of finite type over $O_{K}, i: \bar{X} \hookrightarrow P$ is a closed immersion over $O_{K}, j: X \hookrightarrow \bar{X}$ is an open immersion over $k$ such that $P$ is formally smooth over $O_{K}$ on a neighborhood of $X$. We say that the frame encloses a pair $(X, \bar{X})$.

Definition $3.2([10,4.2 .1])$. A small frame is a frame $(X, \bar{X}, P, i, j)$ such that $\bar{X}$ is isomorphic to $P_{k}$ via $i$ and that there exists an element $f \in \Gamma\left(\bar{X}, \mathcal{O}_{\bar{X}}\right)$ with $X=\{f \neq 0\}$.

Before we introduce some more terminologies on frames, we introduce one terminology which is not standard: For a scheme $Z$, a decomposition of $Z$ into irreducible components or empty schemes is a decomposition $Z=\bigcup_{i \in I} Z_{i}$ such that $Z=\bigcup_{\substack{i \in I \\ Z \\ Z} \emptyset} Z_{i}$ gives the decomposition of $Z$ into irreducible components.

Definition 3.3. Let $X \hookrightarrow \bar{X}$ be an open immersion of smooth $k$-varieties such that $Z:=\bar{X}-X$ is a simple normal crossing divisor. Let $Z=\bigcup_{i=1}^{r} Z_{i}$ be a 
decomposition of $Z$ into irreducible components or empty schemes. Then a standard small frame enclosing $(X, \bar{X})$ is a small frame $\mathcal{P}:=(X, \bar{X}, P, i, j)$ enclosing $(X, \bar{X})$ which satisfies the following condition: There exist $t_{1}, \ldots, t_{r} \in \Gamma\left(P, \mathcal{O}_{P}\right)$ such that, if we denote the zero locus of $t_{i}$ in $P$ by $Q_{i}$, each $Q_{i}$ is irreducible (possibly empty) and that $Q=\bigcup_{i=1}^{r} Q_{i}$ is a relative simple normal crossing divisor of $P$ satisfying $Z_{i}=Q_{i} \times_{P} \bar{X}$. We call a pair $\left(\mathcal{P},\left(t_{1}, \ldots, t_{r}\right)\right)$ a charted standard small frame. When $r=1$, we call $\mathcal{P}$ a smooth standard small frame and the pair $\left(\mathcal{P}, t_{1}\right)$ a charted smooth standard small frame.

Next we recall the relation between isocrystals on log convergent site and $\log -\nabla$ modules. This is explained partly in an abstract way in [13], [14], [15] and explained clearly and explicitly in [10].

Let $X \hookrightarrow \bar{X}$ be an open immersion of smooth $k$-varieties such that $Z:=\bar{X}-X$ is a simple normal crossing divisor and let $Z=\bigcup_{i=1}^{r} Z_{i}$ be the decomposition of $Z$ into irreducible components. Let us denote the $\log$ structure on $\bar{X}$ induced by $Z$ by $M$. Let us take a charted standard small frame $\left((X, \bar{X}, P, i, j),\left(t_{1}, \ldots, t_{r}\right)\right)$ enclosing $(X, \bar{X})$ and let $Q_{i}(1 \leq i \leq r)$ be the zero loci of $t_{i}$ in $P$. Then, by [14, 1.2.7], a locally free isocrystal $\mathcal{E}$ on the log convergent site $\left((\bar{X}, M) / O_{K}\right)_{\text {conv }}$ induces in natural way a $\log$ - $\nabla$-module $E_{\mathcal{E}}$ on $P_{K}$ with respect to $t_{1}, \ldots, t_{r}$. Also, an overconvergent isocrystal $\mathcal{E}$ on $(X, \bar{X}) / K$ induces in natural way a $\nabla$-module on some strict neighborhood of ]$X{ }_{P}$ in $P_{K}$. Following [10, 6.3.1], we make the following definition:

Definition 3.4. Let $X \hookrightarrow \bar{X}$ and $(X, \bar{X}, P, i, j)$ be as above. Then a $\log -\nabla$-module $E$ on $P_{K}$ with respect to $t_{1}, \ldots, t_{r}$ is called convergent if the restriction of $E$ to some strict neighborhood of $] X{ }_{P}$ in $P_{K}$ comes from an overconvergent isocrystal on $(X, \bar{X}) / K$.

Then we have the following proposition:

Proposition 3.5 ([10, 6.4.1]). Let the notations be as above. Then the functor $\mathcal{E} \mapsto E_{\mathcal{E}}$ induces an equivalence of categories

$$
\left(\begin{array}{l}
\text { locally free isocrystals } \\
\text { on }\left((\bar{X}, M) / O_{K}\right)_{\text {conv }}
\end{array}\right) \stackrel{=}{\longrightarrow}\left(\begin{array}{l}
\text { convergent log- } \nabla \text {-modules } \\
\text { on } P_{K} \text { w.r.t. } t_{1}, \ldots, t_{r}
\end{array}\right) .
$$

Keep the above notation. (In particular, we take an isocrystal $\mathcal{E}$ on $\left((\bar{X}, M) / O_{K}\right)$ conv and denote the associated log- $\nabla$-module by $E_{\mathcal{E}}$.) Then, by [10, 6.3.4], the multisequence

$$
\left\{\frac{1}{i_{1} ! \cdots i_{r} !}\left(\prod_{j=1}^{r} \prod_{l=1}^{i_{j}-1}\left(t_{j} \frac{\partial}{\partial t_{j}}-l\right)\right)(\mathbf{v})\right\}_{i_{1}, \ldots, i_{r}}
$$

is $\eta$-null for any $\mathbf{v} \in \Gamma\left(P_{K}, E_{\mathcal{E}}\right)$. For a subset $I$ of $\{1, \ldots, r\}$, let us put $Z_{I}:=$ $\bigcap_{i \in I} Z_{i}, Q_{I}:=\bigcap_{i \in I} Q_{i}$. Then, if $Z_{I}$ is non-empty, the sections $t_{i}(i \in I)$ induce the isomorphism $] Z_{I}\left[{ }_{P} \cong\right] Z_{I}\left[{ }_{Q_{I}} \times A_{K}^{|I|}[0,1)\right.$ (where the coordinate of $A_{K}^{|I|}[0,1$ ) is given by 
$\left.t_{i}(i \in I)\right)$ and for any $\mathbf{v} \in \Gamma\left(P_{K}, E_{\mathcal{E}}\right)$ and for any $\eta \in(0,1), a \in(0,1) \cap \Gamma^{*}$, the multisequence

$$
\left\{\frac{1}{\prod_{j \in I} i_{j} !}\left(\prod_{j \in I} \prod_{l=1}^{i_{j}-1}\left(t_{j} \frac{\partial}{\partial t_{j}}-l\right)\right)(\mathbf{v})\right\}_{i_{j}(j \in I)}
$$

is $\eta$-null on $] Z_{I}\left[Q_{I} \times A_{K}^{|I|}[0, a]\right.$. Hence we have the following:

Proposition 3.6. Let the notations be as above. Then, for any $I \subseteq\{1, \ldots, r\}$, the restriction of $E_{\mathcal{E}}$ to $] Z_{I}[P \cong] Z_{I}\left[Q_{I} \times A_{K}^{|I|}[0,1)\right.$ is log-convergent.

Next we define the notion of 'having exponents in $\Sigma$ ' for an isocrystal on log convergent site.

Definition 3.7. Let $X \hookrightarrow \bar{X}$ be an open immersion of smooth $k$-varieties such that $Z:=\bar{X}-X$ is a simple normal crossing divisor and let $Z=\bigcup_{i=1}^{r} Z_{i}$ be the decomposition of $Z$ by irreducible components. Let us denote the log structure on $\bar{X}$ induced by $Z$ by $M$. Let $\Sigma=\prod_{i=1}^{r} \Sigma_{i}$ be a subset of $\mathbb{Z}_{p}^{r}$. Then we say that a locally free isocrystal $\mathcal{E}$ on $\left((\bar{X}, M) / O_{K}\right)_{\mathrm{conv}}$ has exponents in $\Sigma$ if there exist an affine open covering $\bar{X}=\bigcup_{\alpha \in \Delta} \bar{U}_{\alpha}$ and charted standard small frames $\left(\left(U_{\alpha}, \bar{U}_{\alpha}, P_{\alpha}, i_{\alpha}, j_{\alpha}\right),\left(t_{\alpha, 1}, \ldots, t_{\alpha, r}\right)\right)$ enclosing $\left(U_{\alpha}, \bar{U}_{\alpha}\right)\left(\alpha \in \Delta\right.$, where we put $U_{\alpha}:=$ $\left.X \cap \bar{U}_{\alpha}\right)$ such that, for any $\alpha \in \Delta$ and any $i(1 \leq i \leq r)$, all the exponents of the $\log$ - $\nabla$-module $E_{\mathcal{E}, \alpha}$ on $P_{\alpha, K}$ induced by $\mathcal{E}$ along the locus $\left\{t_{\alpha, i}=0\right\}$ are contained in $\Sigma_{i}$

Then we have the following:

Lemma 3.8. Let $(X, \bar{X}), Z, \Sigma$ be as above. Then a locally free isocrystal $\mathcal{E}$ on $\left((\bar{X}, M) / O_{K}\right)_{\text {conv }}$ has exponents in $\Sigma$ if and only if the following condition is satisfied: For any affine open subscheme $\bar{U} \hookrightarrow \bar{X}$ and any charted standard small frames $\left((U, \bar{U}, P, i, j),\left(t_{1}, \ldots, t_{r}\right)\right)$ enclosing $(U, \bar{U})$ (where we put $\left.U:=X \cap \bar{U}\right)$ such that, for any $i(1 \leq i \leq r)$, all the exponents of the $\log -\nabla$-module $E_{\mathcal{E}}$ on $P_{K}$ induced by $\mathcal{E}$ along the locus $\left\{t_{i}=0\right\}$ are contained in $\Sigma_{i}$.

Proof. Let $\mathcal{E}$ be a locally free isocrystal $\mathcal{E}$ on $\left((\bar{X}, M) / O_{K}\right)_{\text {conv }}$ with exponents in $\Sigma$ and take $\left((U, \bar{U}, P, i, j),\left(t_{1}, \ldots, t_{r}\right)\right), E_{\mathcal{E}}$ as in the statement of the lemma. It suffices to prove that the exponents of $E_{\mathcal{E}}$ along the locus $\left\{t_{i}=0\right\}$ are contained in $\Sigma_{i}$. Note that we may work Zariski locally on $P$.

Let $\bar{X}=\bigcup_{\alpha \in \Delta} \bar{U}_{\alpha}$ and take $\left(\left(U_{\alpha}, \bar{U}_{\alpha}, P_{\alpha}, i_{\alpha}, j_{\alpha}\right),\left(t_{\alpha, 1}, \ldots, t_{\alpha, r}\right)\right)$ and $E_{\mathcal{E}, \alpha}$ as in Definition 3.7. Then, by shrinking $P$, we may assume that $\bar{U}$ is contained in $\bar{U}_{\alpha}$ for some $\alpha$. By shrinking $P_{\alpha}$, we see that we may assume $\bar{U}=\bar{U}_{\alpha}$ to prove the lemma. Moreover, by omitting the indices $i$ with $Z_{i} \cap \bar{U}=\emptyset$, we may assume that $Z_{i} \cap \bar{U} \neq \emptyset$ for any $1 \leq i \leq r$. Under this assumption, we prove that the exponents of $E_{\mathcal{E}}$ and those of $E_{\mathcal{E}, \alpha}$ coincide. 
Let us consider $P$ (resp. $P_{\alpha}$ ) as $\log$ formal scheme with $\log$ structure induced by $\left\{t_{1} \cdots t_{r}=0\right\}$ (resp. $\left\{t_{\alpha, 1} \cdots t_{\alpha, r}=0\right\}$ ) and consider $\bar{U}=\bar{U}_{\alpha}$ as log scheme with log structure induced by $\bar{U} \cap Z$. Then we have the following diagram

$$
\left.P_{\alpha, K}=\right] \bar{U}\left[P_{\alpha} \stackrel{\pi_{1}}{\stackrel{U}{\leftrightarrows}}\right]\left[_ { P _ { \alpha } \times P } ^ { \operatorname { l o g } \cong ] } [ { } _ { \hat { \mathbb { A } } ^ { d } \times P } \stackrel { \pi _ { 2 } } { \longrightarrow } ] \overline { U } \left[{ }_{P}=P_{K},\right.\right.
$$

where $\pi_{i}(i=1,2)$ are the maps induced by the projections, $d=\operatorname{dim} \bar{U}$ and the middle isomorphism is locally defined as follows: Let us choose local parameter of the form $t_{1}, \ldots, t_{d}$ (resp. $t_{\alpha, 1}, \cdots t_{\alpha, d}$ ) of $P$ (resp. $P_{\alpha}$ ), where $t_{i}, t_{\alpha, i}(1 \leq i \leq r)$ are the ones in the data of the charted standard small frames taken above. Let us put

$$
\left(P_{\alpha} \times P\right)^{\prime}:=\left(P_{\alpha} \times P\right) \times_{\operatorname{Spf} O_{K}\left\{t_{1}, \ldots, t_{r}, t_{\alpha, 1}, \ldots, t_{\alpha, r}\right\}} \operatorname{Spf} O_{K}\left\{t_{1}, \ldots, t_{r}, u_{1}, \ldots, u_{r}\right\},
$$

where the left hand side is induced by the map $\operatorname{Spf} O_{K}\left\{t_{1}, \ldots, t_{r}, u_{1}, \ldots, u_{r}\right\} \longrightarrow$ Spf $O_{K}\left\{t_{1}, \ldots, t_{r}, t_{\alpha, 1}, \ldots, t_{\alpha, r}\right\}$ defined by $t_{\alpha, i} \mapsto t_{i}\left(1+u_{i}\right)$. Then we have $] \bar{U}{ }_{P_{\alpha} \times P}^{\log }=$ ] $\bar{U}\left[\left(P_{\alpha} \times P\right)^{\prime}\right.$ by definition and $\left(P_{\alpha} \times P\right)^{\prime}$ is formally smooth over $P$ with relative coordinate $u_{1}, \ldots, u_{r}, t_{r+1}-t_{\alpha, r+1}, \cdots, t_{d}-t_{\alpha, d}$. So we have the isomorphism $] \bar{U}{ }_{P_{\alpha} \times P}^{\log } \cong$

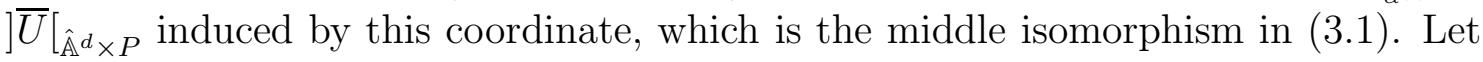
us take the section $s:] \bar{U}\left[_{P} \longrightarrow\right] \bar{U}\left[\hat{\mathbb{A}}^{r \times P}\right.$ of $\pi_{2}$ defined by 'the inclusion into the origin'. Then, by definition, we see that the $\log -\nabla$-module $E_{\mathcal{E}, \alpha}$ restricts to $E_{\mathcal{E}}$ by $\pi_{1} \circ s$. Moreover, by $\pi_{1} \circ s, \operatorname{dlog} t_{\alpha, i}$ is restricted to $s^{*}\left(\operatorname{dlog} t_{i}+\operatorname{dlog}\left(1+u_{i}\right)\right)=\operatorname{dlog} t_{i}$. Hence we see that the residue of $E_{\mathcal{E}, \alpha}$ along $\left\{t_{\alpha, i}=0\right\}$ restricts to the residue of $E_{\mathcal{E}, \alpha}$ along $\left\{t_{i}=0\right\}$ via $\pi_{1} \circ s$. Hence their exponents coincide and so we are done.

Let $X \hookrightarrow \bar{X}$ be an open immersion of smooth $k$-varieties such that $Z:=\bar{X}-X$ is a smooth divisor. Suppose we are given a charted smooth standard small frame $((X, \bar{X}, P, i, j), t)$ enclosing $(X, \bar{X})$ and let $Q$ be the zero loci of $t$ in $P$. Then, as we saw above, an overconvergent isocrystal $\mathcal{E}$ on $(X, \bar{X}) / K$ induces in natural way a $\nabla$-module $E_{\mathcal{E}}$ on some strict neighborhood of $] X{ }_{P}$ in $P_{K}$. In particular, it is defined on $\left\{x \in P_{K}|| t(x) \mid \geq \lambda\right\}$ for some $\lambda \in(0,1) \cap \Gamma^{*}$. Hence we can restrict $E_{\mathcal{E}}$ to $\left.\left\{x \in P_{K}|| t(x) \mid \geq \lambda\right\} \cap\right] Z\left[{ }_{P} \cong\right] Z{ }_{Q} \times A_{K}^{1}[\lambda, 1)=Q_{K} \times A_{K}^{1}[\lambda, 1)$ if $Z$ is non-empty. Using this observation, we define the notion of 'having $\Sigma$-unipotent monodromy' for an overconvergent isocrystal as follows:

Definition 3.9. Let $X \hookrightarrow \bar{X}$ be an open immersion of smooth $k$-varieties such that $Z:=\bar{X}-X$ is a simple normal crossing divisor. Let $Z=\bigcup_{i=1}^{r} Z_{i}$ be the decomposition of $Z$ into irreducible components and let $Z_{\operatorname{sing}}$ be the set of singular points of $Z$. Let $\Sigma=\prod_{i=1}^{r} \Sigma_{i}$ be a subset of $\mathbb{Z}_{p}^{r}$. Then we say that an overconvergent isocrystal $\mathcal{E}$ on $(X, \bar{X}) / K$ has $\Sigma$-unipotent monodromy if there exist an affine open covering $\bar{X}-Z_{\text {sing }}=\bigcup_{\alpha \in \Delta} \bar{U}_{\alpha}$ and charted smooth standard small frames $\left(\left(U_{\alpha}, \bar{U}_{\alpha}, P_{\alpha}, i_{\alpha}, j_{\alpha}\right), t_{\alpha}\right)$ enclosing $\left(U_{\alpha}, \bar{U}_{\alpha}\right)\left(\alpha \in \Delta\right.$, where we put $\left.U_{\alpha}:=X \cap \bar{U}_{\alpha}\right)$ such that, for any $\alpha \in \Delta$, there exists some $\lambda \in(0,1) \cap \Gamma^{*}$ such that the $\nabla$-module $E_{\mathcal{E}, \alpha}$ associated to $\mathcal{E}$ is defined on $\left\{x \in P_{\alpha, K}|| t_{\alpha}(x) \mid \geq \lambda\right\}$ and that the restriction of $E_{\mathcal{E}, \alpha}$ to $Q_{\alpha, K} \times A_{K}^{1}[\lambda, 1)$ is $\Sigma_{i}$-unipotent (where $Q_{\alpha}$ is the zero locus of $t_{\alpha}$ in $P_{\alpha}$ and $i$ is any index with $\bar{U}_{\alpha} \cap Z \subseteq Z_{i}$, which is unique if $\bar{U}_{\alpha} \cap Z$ is non-empty. When $\bar{U}_{\alpha} \cap Z$ is empty, we regard this last condition as vacuous one.) 
Note that the notion of having $\Sigma$-unipotent monodromy depends only on the image $\bar{\Sigma}$ of $\Sigma$ in $\left(\mathbb{Z}_{p} / \mathbb{Z}\right)^{r}$ in the sense that $\mathcal{E}$ has $\Sigma$-unipotent monodromy if and only if $\mathcal{E}$ has $\tau(\bar{\Sigma})$-unipotent monodromy for some (any) section $\tau:\left(\mathbb{Z}_{p} / \mathbb{Z}\right)^{r} \longrightarrow \mathbb{Z}_{p}$ of the canonical projection $\mathbb{Z}_{p}^{r} \longrightarrow\left(\mathbb{Z}_{p} / \mathbb{Z}\right)^{r}$. (See Remark 1.4) Hence we will say also that $\mathcal{E}$ has $\bar{\Sigma}$-unipotent monodromy, by abuse of terminology.

The notion of having $\Sigma$-unipotent monodromy does not depend on the choice of the data chosen in Definition 3.9, under some assumption on $\Sigma$. In fact, we have the following lemma:

Lemma 3.10. Let $(X, \bar{X}), Z=\bigcup_{i=1}^{r} Z_{i}, \Sigma$ be as above and assume that $\Sigma$ is (NID) and $(\mathrm{NLD})$. Then an overconvergent isocrystal $\mathcal{E}$ on $(X, \bar{X}) / K$ has $\Sigma$-unipotent monodromy if and only if the following condition is satisfied: For any affine open subscheme $\bar{U} \hookrightarrow \bar{X}-Z_{\text {sing }}$ and any charted smooth standard small frame $((U, \bar{U}, P, i, j), t)$ enclosing $(U, \bar{U})$ (where we put $U:=X \cap \bar{U})$, there exists some $\lambda \in(0,1) \cap \Gamma^{*}$ such that the $\nabla$-module $E_{\mathcal{E}}$ associated to $\mathcal{E}$ is defined on $\left\{x \in P_{K}|| t(x) \mid \geq \lambda\right\}$ and that the restriction of $E_{\mathcal{E}}$ to $Q_{K} \times A_{K}^{1}[\lambda, 1)$ is $\Sigma_{i}$-unipotent (where $Q$ is the zero locus of t in $P$ and $i$ is any index with $\bar{U} \cap Z \subseteq Z_{i}$, which is unique if $\bar{U} \cap Z$ is non-empty. When $\bar{U} \cap Z$ is empty, we regard this last condition as vacuous one.)

Proof. Let $\mathcal{E}$ be an overconvegent isocrystal on $(X, \bar{X}) / K$ having $\Sigma$-unipotent monodromy, and let $((U, \bar{U}, P, i, j), t), Q$ and $E_{\mathcal{E}}$ (resp. $\bar{X}-Z_{\text {sing }}=\bigcup_{\alpha \in \Delta} \bar{U}_{\alpha},\left(\left(U_{\alpha}, \bar{U}_{\alpha}\right.\right.$, $\left.\left.P_{\alpha}, i_{\alpha}, j_{\alpha}\right), t_{\alpha}\right), Q_{\alpha}$ and $\left.E_{\mathcal{E}, \alpha}\right)$ as in the statement of the lemma (resp. Definition 3.9). It suffices to prove that, under the assumption $\bar{U}=\bar{U}_{\alpha}$ for some $\alpha$ and the assumption $\bar{U} \cap Z \neq \emptyset$, the restriction to $E_{\mathcal{E}}$ to $Q_{K} \times A_{K}^{1}[\lambda, 1)$ is $\Sigma_{i}$-unipotent for some $\lambda$. (Here $i$ is the index with $\bar{U} \cap Z \subset Z_{i}$.)

Let $M$ be the $\log$ structure on $\bar{X}$ induced by $Z$. By assumption, $E_{\mathcal{E}, \alpha}$ is defined on $\left\{x \in P_{\alpha, K}|| t_{\alpha}(x) \mid \geq \lambda\right\}$ for some $\lambda$ and $\Sigma_{i}$-unipotent on $\left\{x \in P_{\alpha, K}|| t_{\alpha}(x) \mid \geq\right.$ $\lambda\} \cap] Z \cap \bar{U}_{\alpha}\left[P_{\alpha} \cong Q_{\alpha, K} \times A_{K}^{1}[\lambda, 1)\right.$. Then, by Corollary 1.15, we may suppose that the restriction of $E_{\mathcal{E}, \alpha}$ to $Q_{\alpha, K} \times A_{K}^{1}[\lambda, 1)$ extends to a $\log$ - $\nabla$-module on $Q_{\alpha, K} \times$ $A_{K}^{1}[0,1)$ with exponents in $\Sigma_{i}$. Therefore, $E_{\mathcal{E}, \alpha}$ extends to a $\log -\nabla$-module on $P_{\alpha, K}$ with respect to $t_{\alpha}$ with exponents in $\Sigma_{i}$, that is, $\mathcal{E}$ extends to an isocrystal $\widetilde{\mathcal{E}}$ on $\left(\left(\bar{U},\left.M\right|_{\bar{U}}\right) / O_{K}\right)_{\text {conv }}$ with exponents in $\Sigma_{i}$. Then $\widetilde{\mathcal{E}}$ induces a $\log -\nabla$-module $E_{\widetilde{\mathcal{E}}}$ on $P_{K}$ with respect to $t$ with exponents in $\Sigma_{i}$ which extends $E_{\mathcal{E}}$. Then the restriction of $E_{\widetilde{\mathcal{E}}}$ to $Q_{K} \times A_{K}^{1}[0,1)$ is log-convergent and has exponents in $\Sigma_{i}$. Hence it is $\Sigma_{i^{-}}$ unipotent by Propsition 2.12. Hence the restriction of $E_{\widetilde{\mathcal{E}}}$ to $Q_{K} \times A_{K}^{1}[\lambda, 1)$, which is nothing but the restriction of $E_{\mathcal{E}}$ to $Q_{K} \times A_{K}^{1}[\lambda, 1)$ if $\lambda$ is sufficiently close to 1 , is also $\Sigma_{i}$-unipotent. So we are done.

We make the following auxiliary definition to prove that the property of 'having $\Sigma$-unipotent monodromy' is generic in some sense:

Definition 3.11. Let $X \hookrightarrow \bar{X}$ be an open immersion of smooth $k$-varieties such that $Z:=\bar{X}-X$ is a simple normal crossing divisor. Let $Z=\bigcup_{i=1}^{r} Z_{i}$ be the decomposition of $Z$ by irreducible components and let $Z_{\operatorname{sing}}$ be the set of singular 
points of $Z$. Let $\Sigma=\prod_{i=1}^{r} \Sigma_{i}$ be a subset of $\mathbb{Z}_{p}^{r}$. Then we say that an overconvergent isocrystal $\mathcal{E}$ on $(X, \bar{X}) / K$ has $\Sigma$-unipotent generic monodromy if there exist affine open subschemes $\bar{U}_{\alpha} \subseteq \bar{X}-Z_{\text {sing }}$ containing the generic point of $Z_{\alpha}(1 \leq \alpha \leq r)$, charted smooth standard small frames $\left(\left(U_{\alpha}, \bar{U}_{\alpha}, P_{\alpha}, i_{\alpha}, j_{\alpha}\right), t_{\alpha}\right)$ enclosing $\left(U_{\alpha}, \bar{U}_{\alpha}\right)$ (where we put $\left.U_{\alpha}:=X \cap \bar{U}_{\alpha}\right)$, fields $L_{\alpha}$ containing $\Gamma\left(Q_{\alpha, K}, \mathcal{O}\right)$ (where $Q_{\alpha}$ is the zero locus of $t_{\alpha}$ in $P_{\alpha}$ ) which is complete with respect to a norm which restricts to the supremum norm on $Q_{\alpha, K}$ satisfying the following condition: For any $1 \leq \alpha \leq r$, there exists some $\lambda \in(0,1) \cap \Gamma^{*}$ such that the $\nabla$-module $E_{\mathcal{E}, \alpha}$ associated to $\mathcal{E}$ is defined on $\left\{x \in P_{\alpha, K}|| t_{\alpha}(x) \mid \geq \lambda\right\}$ and that the restriction of $E_{\mathcal{E}, \alpha}$ to $A_{L_{\alpha}}^{1}[\lambda, 1)$ is $\Sigma_{\alpha}$-unipotent.

Note that the notion of having $\Sigma$-unipotent monodromy depends only on the image $\bar{\Sigma}$ of $\Sigma$ in $\left(\mathbb{Z}_{p} / \mathbb{Z}\right)^{r}$ in the sense that $\mathcal{E}$ has $\Sigma$-unipotent monodromy if and only if $\mathcal{E}$ has $\tau(\bar{\Sigma})$-unipotent monodromy for some (or any) section $\tau:\left(\mathbb{Z}_{p} / \mathbb{Z}\right)^{r} \longrightarrow \mathbb{Z}_{p}$ of the canonical projection $\mathbb{Z}_{p}^{r} \longrightarrow\left(\mathbb{Z}_{p} / \mathbb{Z}\right)^{r}$. (See Remark 1.4.) Hence, as in the case of $\Sigma$-unipotent monodromy, it is allowed to say also that $\mathcal{E}$ has $\bar{\Sigma}$-unipotent generic monodromy by abuse of terminology, where $\bar{\Sigma}$ is the image of $\Sigma$ in $\left(\mathbb{Z}_{p} / \mathbb{Z}\right)^{r}$.

As for the relation between monodromy and generic monodromy, we have the following:

Proposition 3.12. Let $(X, \bar{X}), Z=\bigcup_{i=1}^{r} Z_{i}, \Sigma$ be as above and assume that $\Sigma$ is (NID) and (NLD). Then an overconvergent isocrystal $\mathcal{E}$ on $(X, \bar{X}) / K$ has $\Sigma$ unipotent monodromy if and only if it has $\Sigma$-unipotent generic monodromy.

Proof. It suffices to prove that 'having $\Sigma$-unipotent generic monodromy' implies 'having $\Sigma$-unipotent monodromy'. Let $\mathcal{E}$ be an overconvergent isocrystal on $(X, \bar{X})$ $/ K$ which has $\Sigma$-unipotent generic monodromy, and let us take an affine open subscheme $\bar{U} \hookrightarrow \bar{X}-Z_{\text {sing }}$ and a charted smooth standard small frame $(\mathcal{P}:=$ $(U, \bar{U}, P, i, j), t)$ enclosing $(U, \bar{U})$ (where $U:=X \cap \bar{U})$ with $\bar{U} \cap Z \neq \emptyset$. Let $Q$ be the zero locus of $t$ in $P$ and let $i$ be the index with $\bar{U} \cap Z \subseteq Z_{i}$. Let $E_{\mathcal{E}}$ be a $\nabla$-module on a strict neighborhood of $] U\left[{ }_{P}\right.$ in $P_{K}$ induced by $\mathcal{E}$. It suffices to prove that there exists some $\lambda \in(0,1) \cap \Gamma^{*}$ such that $E_{\mathcal{E}}$ is defined on $\left\{x \in P_{K}|| t(x) \mid \geq \lambda\right\}$ and that the restriction of $E_{\mathcal{E}}$ to $Q_{K} \times A_{K}^{1}[\lambda, 1)$ is $\Sigma_{i}$-unipotent. First let us note that, in the case where $(\mathcal{P}, t)$ is equal to $\left(\mathcal{P}_{\alpha}:=\left(U_{\alpha}, \bar{U}_{\alpha}, P_{\alpha}, i_{\alpha}, j_{\alpha}\right), t_{\alpha}\right)$ in Definition 3.11, this claim is true (with $i=\alpha$ ) by Proposition 2.4.

Now we prove the claim in the previous paragraph in general case. Since $\bar{U} \cap Z$ is non-empty, $\bar{U}$ has non-empty intersection with some $\bar{U}_{\alpha}$. Then, if we replace $\bar{U}$ by a smaller affine open subscheme which is contained also in $\bar{U}_{\alpha}$, the claim is true for $(\mathcal{P}, t)$ by the fact the claim is true for the restriction of $\left(\mathcal{P}_{\alpha}, t_{\alpha}\right)$ to $\bar{U}$ and by Lemma 3.10. Then we see that the claim is true for general $\bar{U}$ and $(\mathcal{P}, t)$, by Proposition 2.4.

Next we introduce the notion of the Robba condition for an overconvergent isocrystal. 
Definition 3.13. Let $X \hookrightarrow \bar{X}$ be an open immersion of smooth $k$-varieties such that $Z:=\bar{X}-X$ is a simple normal crossing divisor. Let $Z=\bigcup_{i=1}^{r} Z_{i}$ be the decomposition of $Z$ by irreducible components and let $Z_{\operatorname{sing}}$ be the set of singular points of $Z$. Then we say that an overconvergent isocrystal $\mathcal{E}$ on $(X, \bar{X}) / K$ satisfies the Robba condition if it satisfies the following condition: There exist affine open subschemes $\bar{U}_{\alpha} \subseteq \bar{X}-Z_{\text {sing }}$ containing the generic point of $Z_{\alpha}(1 \leq \alpha \leq r)$, charted smooth standard small frames $\left(\mathcal{P}_{\alpha}:=\left(U_{\alpha}, \bar{U}_{\alpha}, P_{\alpha}, i_{\alpha}, j_{\alpha}\right), t_{\alpha}\right)$ enclosing $\left(U_{\alpha}, \bar{U}_{\alpha}\right)$ (where we put $\left.U_{\alpha}:=X \cap \bar{U}_{\alpha}\right)$, fields $L_{\alpha}$ containing $\Gamma\left(Q_{\alpha, K}, \mathcal{O}\right)$ (where $Q_{\alpha}$ is the zero locus of $t_{\alpha}$ in $\left.P_{\alpha}\right)$ which are complete with respect to a norm which restricts to the spectral norm on $Q_{\alpha, K}$ satisfying the following condition: For any $1 \leq \alpha \leq r$, there exists some $\lambda \in(0,1) \cap \Gamma^{*}$ such that the $\nabla$-module $E_{\mathcal{E}, \alpha}$ associated to $\mathcal{E}$ is defined on $\left\{x \in P_{\alpha, K}|| t_{\alpha}(x) \mid \geq \lambda\right\}$ and that the restriction $F_{\mathcal{E}, \alpha}$ of $E_{\mathcal{E}, \alpha}$ to $A_{L_{\alpha}}^{1}[\lambda, 1)$ satisfies the Robba condition in the sense of [7, 11.1].

Let the notation be as in Definition 3.13 and let $\mu$ be the rank of $\mathcal{E}$. Then, for each $1 \leq \alpha \leq r$, we have the notion of an exponent $\operatorname{Exp}\left(F_{\mathcal{E}, \alpha}\right)$ of $F_{\mathcal{E}, \alpha}$ in the sense of [7, 11.4], which is an element in $\mathbb{Z}_{p}^{\mu} / \stackrel{e}{\sim}$, where $\stackrel{e}{\sim}$ is a certain equivalent relation (defined in [7, 10.4]). We call an overconvergent isocrystal $\mathcal{E}$ on $(X, \bar{X}) / K$ satisfying the Robba condition is (NLD) if, for each $1 \leq \alpha \leq r$, the difference of any two components of any lift of $\operatorname{Exp}\left(F_{\mathcal{E}, \alpha}\right)$ to $\mathbb{Z}_{p}^{\mu}$ is $p$-adically non-Liouville.

Let the situation be as above and assume that $\mathcal{E}$ is an overconvergent isocrystal $(X, \bar{X}) / K$ which satisfies the Robba condition and which is (NLD). In this case, it is known $([7,10.5])$ that $\operatorname{Exp}\left(F_{\mathcal{E}, \alpha}\right)$ is well-defined as an element of $\left(\mathbb{Z}_{p} / \mathbb{Z}\right)^{\mu}$ up to order. So, if we take a representative $\left(a_{1}, \ldots, a_{\mu}\right) \in\left(\mathbb{Z}_{p} / \mathbb{Z}\right)^{\mu}$ of $\operatorname{Exp}\left(F_{\mathcal{E}, \alpha}\right)$, the set $\bar{\Sigma}_{\mathcal{E}, \alpha}:=\left\{a_{1}, \ldots, a_{\mu}\right\} \subseteq \mathbb{Z}_{p} / \mathbb{Z}$ does not depend on the representative. Let us put $\bar{\Sigma}_{\mathcal{E}}:=\prod_{\alpha=1}^{r} \bar{\Sigma}_{\mathcal{E}, \alpha} \subseteq\left(\mathbb{Z}_{p} / \mathbb{Z}\right)^{r}$. Then, by [6, 6-2.6] and [7, 12.1], we see that $\mathcal{E}$ has $\bar{\Sigma}_{\mathcal{E}}$-unipotent generic monodromy. Noting the fact that $\bar{\Sigma}_{\mathcal{E}}$ is (NLD) (hence $\tau\left(\bar{\Sigma}_{\mathcal{E}}\right)$ is (NLD) and (NLD) for any section $\tau:\left(\mathbb{Z}_{p} / \mathbb{Z}\right)^{r} \longrightarrow \mathbb{Z}_{p}^{r}$ of the canonical projection) and using Proposition 3.12, we have the following:

Proposition 3.14. Let $X, \bar{X}$ be as above and let $\mathcal{E}$ be an overconvergent isocrystal on $(X, \bar{X}) / K$ which satisfies the Robba condition and which is (NLD). Then, if we define $\bar{\Sigma}_{\mathcal{E}} \subseteq\left(\mathbb{Z}_{p} / \mathbb{Z}\right)^{r}$ as above, $\mathcal{E}$ has $\bar{\Sigma}_{\mathcal{E}}$-unipotent monodromy.

Remark 3.15. On the other hand, we can prove directly that an overconvergent isocrystal $\mathcal{E}$ having $\Sigma$-unipotent generic monodromy for some $\Sigma$ which is (NID) and (NLD) satisfies the Robba condition and in this case, $\bar{\Sigma}_{\mathcal{E}}$ is contained in the image of $\Sigma$ in $\left(\mathbb{Z}_{p} / \mathbb{Z}\right)^{r}$.

Now we state and prove the main result in this paper, which says that, under certain condition, overconvergent isocrystals having $\Sigma$-unipotent monodromy extends uniquely to an isocrystal on log convergent site with exponents in $\Sigma$ :

Theorem 3.16. Let $X \hookrightarrow \bar{X}$ be an open immersion of smooth $k$-varieties such that $Z:=\bar{X}-X$ is a simple normal crossing divisor and let $Z=\bigcup_{i=1}^{r} Z_{i}$ be the 
decomposition of $Z$ into irreducible components. Let us denote the log structure on $X$ induced by $Z$ by $M$. Let $\Sigma:=\prod_{i=1}^{r} \Sigma_{i}$ be a subset of $\mathbb{Z}_{p}^{r}$ which is (NID) and (NLD). Then we have the canonical equivalence of categories

$$
j^{\dagger}:\left(\begin{array}{l}
\text { isocrystals on } \\
\left((\bar{X}, M) / O_{K}\right)_{\text {conv }} \\
\text { with exponents in } \Sigma
\end{array}\right) \stackrel{=}{\longrightarrow}\left(\begin{array}{l}
\text { overcongent isocrystals } \\
\text { on }(X, \bar{X}) / K \text { having } \\
\Sigma \text {-unipotent monodromy }
\end{array}\right),
$$

which is defined by the restriction.

Remark 3.17. By using the image of $\bar{\Sigma}$ of $\Sigma$ in $\left(\mathbb{Z}_{p} / \mathbb{Z}\right)^{r}$ instead of $\Sigma$, we can express Theorem 3.16 in the following way: Let $X \hookrightarrow \bar{X}, Z=\bigcup_{i=1}^{r} Z_{i}$ and $M$ be as above. Let Let $\bar{\Sigma}:=\prod_{i=1}^{r} \bar{\Sigma}_{i}$ be a subset of $\left(\mathbb{Z}_{p} / \mathbb{Z}\right)^{r}$ which is (NLD). Then, for each section $\tau:\left(\mathbb{Z}_{p} / \mathbb{Z}\right)^{r} \longrightarrow \mathbb{Z}_{p}$ of the canonical projection $\mathbb{Z}_{p}^{r} \longrightarrow\left(\mathbb{Z}_{p} / \mathbb{Z}\right)^{r}$, we have the canonical equivalence of categories

$$
j^{\dagger}:\left(\begin{array}{l}
\text { isocrystals on } \\
\left((\bar{X}, M) / O_{K}\right)_{\text {conv }} \\
\text { with exponents in } \tau(\bar{\Sigma})
\end{array}\right) \stackrel{=}{\longrightarrow}\left(\begin{array}{l}
\text { overcongent isocrystals } \\
\text { on }(X, \bar{X}) / K \text { having } \\
\bar{\Sigma} \text {-unipotent monodromy }
\end{array}\right),
$$

which is defined by the restriction.

Before the proof of Theorem [3.16, we state an immediate corollary, which is regarded as a $p$-adic analogue of [8, II 5.4]:

Corollary 3.18. Let $X \subseteq \bar{X}, Z=\bigcup_{i=1}^{r} Z_{i}$ and $M$ be as above and let $\mathcal{E}$ be an overconvergent isocrystal on $(X, \bar{X}) / K$ which satisfies the Robba condition and which is (NLD). Then, for each $\tau:\left(\mathbb{Z}_{p} / \mathbb{Z}\right)^{r} \longrightarrow \mathbb{Z}_{p}$ of the canonical projection $\mathbb{Z}_{p}^{r} \longrightarrow$ $\left(\mathbb{Z}_{p} / \mathbb{Z}\right)^{r}, \mathcal{E}$ extends uniquely and canonically to an isocrystal on $\left((\bar{X}, M) / O_{K}\right)_{\mathrm{conv}}$ with exponents in $\tau\left(\bar{\Sigma}_{\mathcal{E}}\right)$, where $\bar{\Sigma}_{\mathcal{E}}$ is the subset of $\left(\mathbb{Z}_{p} / \mathbb{Z}\right)^{r}$ defined in the paragraph before Proposition 3.14 .

Proof. Immediate from Theorem 3.16 and Proposition 3.14 .

Now we give a proof of Theorem 3.16:

Proof of Theorem 3.16. First let us see tht the functor is well-defined, that is, for an isocrystal $\mathcal{E}$ on $\left((\bar{X}, M) / O_{K}\right)$ conv with exponents in $\Sigma$, the restriction $j^{\dagger} \mathcal{E}$ of $\mathcal{E}$ to the category of overconvergent isocrystal on $(X, \bar{X}) / K$ has $\Sigma$-unipotent monodromy. To see this, let us take an affine open subscheme $\bar{U} \hookrightarrow \bar{X}-Z_{\text {sing }}$ and a charted smooth standard small frame $((U, \bar{U}, P, i, j), t)$ enclosing $(U, \bar{U})$ (where $U:=X \cap \bar{U}$ ) with $\bar{U} \cap Z \neq \emptyset$. Let $Q$ be the zero locus of $t$ in $P$. Then $\mathcal{E}$ induces a $\log -\nabla$-module $E_{\mathcal{E}}$ on $P_{K}$ with respect to $t$ whose exponents along $Q_{K}$ is in $\Sigma_{i}$, where $i$ is the index with $\bar{U} \cap Z \subseteq Z_{i}$. Then the restriction of $E_{\mathcal{E}}$ to $] \bar{U} \cap Z\left[{ }_{P}=Q_{K} \times A_{K}^{1}[0,1)\right.$ has exponents in $\Sigma_{i}$ and it is log-convergent. Hence it is $\Sigma$-unipotent by Proposition 2.12 and so is 
the restriction of it to $Q_{K} \times A_{K}^{1}[\lambda, 1)$ for any $\lambda \in(0,1) \cap \Gamma^{*}$. So $j^{\dagger} \mathcal{E}$ has $\Sigma$-unipotent monodromy.

Next we see that, if we are given a morphism $f: \mathcal{E} \longrightarrow \mathcal{F}$ of overconvergent isocrystals on $(X, \bar{X}) / K$ having $\Sigma$-unipotent monodromy and if we have extensions $\widetilde{\mathcal{E}}, \widetilde{\mathcal{F}}$ of $\mathcal{E}, \mathcal{F}$ to isocrystals on $\left((\bar{X}, M) / O_{K}\right)_{\text {conv }}$ with exponents in $\Sigma, f$ extends uniquely to the morphism $\widetilde{f}: \widetilde{\mathcal{E}} \longrightarrow \widetilde{\mathcal{F}}$. To see this, we may work Zariski locally on $\bar{X}$. Hence we may assume that there exists a charted standard small frame $\left((X, \bar{X}, P, i, j),\left(t_{1}, \ldots, t_{r}\right)\right)$ enclosing $(X, \bar{X})$. Let $\varphi: E_{\mathcal{E}} \longrightarrow E_{\mathcal{F}}$ be a morphism of $\nabla$-modules on a strict neighborhood of $] X\left[P\right.$ in $P_{K}$ induced by $f$ and let $E_{\widetilde{\mathcal{E}}}, E_{\widetilde{\mathcal{F}}}$ be the $\log -\nabla$-module on $P_{K}$ with respect to $t_{1}, \ldots, t_{r}$ induced by $\widetilde{\mathcal{E}}, \widetilde{\mathcal{F}}$. Then there exists some $\lambda \in(0,1) \cap \Gamma^{*}$ such that both $E_{\mathcal{E}}, E_{\mathcal{F}}$ are defined on $\mathfrak{Y}:=\left\{x \in P_{K}|\forall i,| t_{i}(x) \mid \geq\right.$ $\lambda\}$. Let us consider the admissible covering $P_{K}=\bigcup_{I \subseteq\{1, \ldots, r\}} \mathfrak{X}_{I}$, where $\mathfrak{X}_{I}$ is defined by

$$
\mathfrak{X}_{I}:=\left\{x \in P_{K}|| t_{i}(x)|<1(i \in I),| t_{i}(x) \mid \geq \lambda(i \notin I)\right\} .
$$

This covering induces the admissible covering $\mathfrak{Y}=\bigcup_{I \subseteq\{1, \ldots, r\}} \mathfrak{Y}{ }_{I}$, where

$$
\mathfrak{Y}_{I}:=\left\{x \in P_{K}|\lambda \leq| t_{i}(x)|<1(i \in I),| t_{i}(x) \mid \geq \lambda(i \notin I)\right\} .
$$

By assumption, $E_{\widetilde{\mathcal{E}}}, E_{\widetilde{\mathcal{F}}}$ have exponents in $\Sigma$ and log-convergent on $\left\{x \in P_{K} \mid t_{i}(x)=\right.$ $0(i \in I)\} \times A^{|I|}[0,1)$. Hence they are $\Sigma$-unipotent on it. Hence they are $\Sigma$-unipotent on

$$
\mathfrak{X}_{I}=\left\{x \in P_{K}\left|t_{i}(x)=0(i \in I),\right| t_{i}(x) \mid \geq \lambda(i \notin I)\right\} \times A^{|I|}[0,1),
$$

which extends the restriction of $E_{\mathcal{E}}, E_{\mathcal{F}}$ to

$$
\mathfrak{Y}_{I}=\left\{x \in P_{K}\left|t_{i}(x)=0(i \in I),\right| t_{i}(x) \mid \geq \lambda(i \notin I)\right\} \times A^{|I|}[\lambda, 1) .
$$

Hence, by Corollary 1.16, there exists a unique morphism $\widetilde{\varphi}: E_{\widetilde{\mathcal{E}}} \longrightarrow E_{\widetilde{\mathcal{F}}}$ on $\mathfrak{X}_{I}$ which extends the map $\varphi$ on $\mathfrak{Y}_{I}$. On

$$
\begin{aligned}
\mathfrak{X}_{I} \cap \mathfrak{X}_{J}=\left\{x \in P_{K}|\lambda \leq| t_{i}(x) \mid<1(i\right. & \in(I \cup J)-(I \cap J)), \\
\lambda & \left.\leq\left|t_{i}(x)\right|(i \notin I \cup J)\right\} \times A_{K}^{|I \cap J|}[0,1),
\end{aligned}
$$

the maps $\widetilde{\varphi}$ we constructed on $\mathfrak{X}_{I}$ and on $\mathfrak{X}_{J}$ coincide because they both extends the $\operatorname{map} \varphi$ on

$$
\begin{aligned}
\mathfrak{Y}_{I} \cap \mathfrak{Y}_{J}=\left\{x \in P_{K}|\lambda \leq| t_{i}(x) \mid<1\right. & (i \in(I \cup J)-(I \cap J)), \\
\lambda & \left.\leq\left|t_{i}(x)\right|(i \notin I \cup J)\right\} \times A_{K}^{|I \cap J|}[\lambda, 1) .
\end{aligned}
$$

Therefore we can glue them and so we have a unique extension $\widetilde{\varphi}: E_{\widetilde{\mathcal{E}}} \longrightarrow E_{\widetilde{\mathcal{F}}}$ of the map $\varphi$ which gives the desired map $\widetilde{f}: \widetilde{\mathcal{E}} \longrightarrow \widetilde{\mathcal{F}}$.

Finally, we prove the essential surjectivity of the functor $j^{\dagger}$, that is, any overconvergent isocrystal $\mathcal{E}$ on $(X, \bar{X}) / K$ having $\Sigma$-unipotent monodromy necessarily 
extends to an isocrystal on $\left((\bar{X}, M) / O_{K}\right)_{\text {conv }}$ with exponents in $\Sigma$. Since we have already shown the uniqueness of the extension, we may work locally on $\bar{X}$, that is, we may assume that there exists a charted standard small frame $\left((X, \bar{X}, P, i, j),\left(t_{1}, \ldots\right.\right.$, $\left.t_{r}\right)$ ) enclosing $(X, \bar{X})$. Let $Q_{i}$ be the zero locus of $t_{i}$ in $P$ and put $Z_{i}^{0}:=Z_{i}-\bigcup_{j \neq i}\left(Z_{i} \cap\right.$ $Z_{j}$ ). By definition, $\mathcal{E}$ induces a $\nabla$-module $E:=E_{\mathcal{E}}$ on $\left\{x \in P_{K}|\forall j,| t_{j}(x) \mid \geq \lambda\right\}$ which is $\Sigma$-unipotent on

$$
\left.\left\{x \in P_{K}|\forall j,| t_{j}(x) \mid \geq \lambda\right\} \cap\right] Z_{i}^{0}\left[{ }_{P}=\left\{x \in Q_{i, K}|\forall j \neq i,| t_{j}(x) \mid=1\right\} \times A_{K}^{1}[\lambda, 1)\right.
$$

for some $\lambda \in(0,1) \cap \Gamma^{*}$.

It suffices to prove that $E$ extends to a $\log -\nabla$-module on $P_{K}$ with respect to $t_{1}, \ldots, t_{r}$ with exponents in $\Sigma$. Hence we are reduced to proving that, for any $0 \leq$ $a \leq r$, there exists some $\lambda$ such that $E$ extends to a $\log -\nabla$-module on $\left\{x \in P_{K} \mid \forall j>\right.$ $\left.a,\left|t_{j}(x)\right| \geq \lambda\right\}$ with respect to $t_{1}, \ldots, t_{r}$ with exponents in $\Sigma$. We prove this claim by induction on $a$. (The case $a=0$ is true by assumption.)

Assume that the claim is true for $a-1$ (for some $\lambda$ ). For any $\lambda^{\prime} \in[\lambda, 1) \cap \Gamma^{*}$, we have an admissible covering $P_{K}=\left\{x \in P_{K}|| t_{a}(x) \mid \geq \lambda^{\prime}\right\} \cup\left\{x \in P_{K}|| t_{a}(x) \mid<1\right\}$. Hence we have the induced admissible coverings

$$
\begin{aligned}
& \left\{x \in P_{K}|\forall j>a-1,| t_{j}(x) \mid \geq \lambda^{\prime}\right\} \\
= & \left\{x \in P_{K}|\forall j>a-1,| t_{j}(x) \mid \geq \lambda^{\prime}\right\} \cup\left\{x \in P_{K}|\forall j>a,| t_{j}(x)\left|\geq \lambda^{\prime}, \lambda^{\prime} \leq\right| t_{a}(x) \mid<1\right\} \\
= & \left\{x \in P_{K}|\forall j>a-1,| t_{j}(x) \mid \geq \lambda^{\prime}\right\} \cup\left(\left\{x \in Q_{a, K}|\forall j>a,| t_{j}(x) \mid \geq \lambda^{\prime}\right\} \times A_{K}^{1}\left[\lambda^{\prime}, 1\right)\right),
\end{aligned}
$$

$$
\begin{aligned}
& \left\{x \in P_{K}|\forall j>a,| t_{j}(x) \mid \geq \lambda^{\prime}\right\} \\
= & \left\{x \in P_{K}|\forall j>a-1,| t_{j}(x) \mid \geq \lambda^{\prime}\right\} \cup\left\{x \in P_{K}|\forall j>a,| t_{j}(x)\left|\geq \lambda^{\prime},\right| t_{a}(x) \mid<1\right\} \\
= & \left\{x \in P_{K}|\forall j>a-1,| t_{j}(x) \mid \geq \lambda^{\prime}\right\} \cup\left(\left\{x \in Q_{a, K}|\forall j>a,| t_{j}(x) \mid \geq \lambda^{\prime}\right\} \times A_{K}^{1}[0,1)\right) .
\end{aligned}
$$

By induction hypothesis, the $\log -\nabla$-module $E$ with exponents in $\Sigma$ is defined on $\left\{x \in P_{K}|\forall j>a-1,| t_{j}(x) \mid \geq \lambda\right\}$ and it is $\Sigma$-unipotent on $\left\{x \in Q_{a, K} \mid \forall j \neq\right.$ $\left.a,\left|t_{j}(x)\right|=1\right\} \times A_{K}^{1}[\lambda, 1)$. By (3.3) and (3.4), we see that it suffices to extend the restriction of $E$ to $\left\{x \in Q_{a, K}|\forall j>a,| t_{j}(x) \mid \geq \lambda^{\prime}\right\} \times A_{K}^{1}\left[\lambda^{\prime}, 1\right)$ to some log- $\nabla$ module on $\left\{x \in Q_{a, K}|\forall j>a,| t_{j}(x) \mid \geq \lambda^{\prime}\right\} \times A_{K}^{1}[0,1)$ with exponents in $\Sigma$, for some $\lambda^{\prime} \in(\lambda, 1) \cap \Gamma^{*}$.

Take any closed aligned subinterval $[b, c] \subseteq(\lambda, 1)$ of positive length. Then, by the $\Sigma$-unipotence of $E$ on $\left\{x \in Q_{a, K}|\forall j \neq a,| t_{j}(x) \mid=1\right\} \times A_{K}^{1}[\lambda, 1)$ and Proposition 2.7. we see that there exists some $\lambda^{\prime} \in(c, 1) \cap \Gamma^{*}$ such that $E$ is $\Sigma$-unipotent on

$$
\left\{x \in Q_{a, K}|\forall j>a,| t_{j}(x)\left|\geq \lambda^{\prime}, \forall j<a,\right| t_{j}(x) \mid=1\right\} \times A_{K}^{1}[b, c] .
$$

Then, by Proposition 2.4, we see that $E$ is $\Sigma$-unipotent on

$$
\left\{x \in Q_{a, K}|\forall j>a,| t_{j}(x) \mid \geq \lambda^{\prime}\right\} \times A_{K}^{1}(b, c) .
$$


So it can be extended to a $\log -\nabla$-module with exponents in $\Sigma$ on

$$
\left\{x \in Q_{a, K}|\forall j>a,| t_{j}(x) \mid \geq \lambda^{\prime}\right\} \times A_{K}^{1}[0, c) .
$$

Hence we can glue it with $E$ on $\left\{x \in Q_{a, K}|\forall j>a,| t_{j}(x) \mid \geq \lambda^{\prime}\right\} \times A_{K}^{1}[\lambda, 1)$ to give a $\log$ - $\nabla$-module with exponents in $\Sigma$ on

$$
\left\{x \in Q_{a, K}|\forall j>a,| t_{j}(x) \mid \geq \lambda^{\prime}\right\} \times A_{K}^{1}[0,1),
$$

as desired. So the proof of the theorem is finished.

\section{References}

[1] F. Baldassarri and B. Chiarellotto, On Christol's theorem. A generalization to systems of PDE's with logarithmic singularities depending upon parameters, Contemporary Math. 133, 1-24.

[2] F. Baldassarri and B. Chiarellotto, Formal and p-adic theory of differential systems with logarithmic singularities depending upon parameters, Duke Math. J. 72(1993), 241-300.

[3] F. Baldassarri and B. Chiarellotto, Algebraic versus rigid Cohomology with logarithmic coefficients, in Barsotti Symposium in Algebraic Geometry, Academic Press.

[4] P. Berthelot, Cohomologie rigide et cohomologie rigide à supports propres première partie, prépublication de l'IRMAR 96-03. Available at http://perso.univ-rennes1.fr/pierre.berthelot/

[5] G. Christol, Un théorème de transfert pour les disques singuliers réguliers, Astérisque 119-120(1984), 151-168.

[6] G. Christol and Z. Mebkhout, Sur le théorème de l'induce des équations différentielles p-adiques II, Ann. of Math. 146(1997), 345-410.

[7] G. Christol and Z. Mebkhout, Équations différentielles p-adiques et coefficients p-adiques sur les courbes, Astérisque 279(2002), 125-183.

[8] P. Deligne, Equations différentielles à points singuliers réguliers, Lecture Note in Math. 163, Springer-Verlag, 1970.

[9] B. Dwork, G. Gerotto and F. J. Sullivan, An introduction to G-functions, Annals of Math. Studies 133, Princeton University Press (1994).

[10] K. S. Kedlaya, Semistable reduction for overconvergent F-isocrystals, I: Unipotence and logarithmic extensions, Compositio Math.. 143(2007), 11641212 . 
[11] K. S. Kedlaya, Semistable reduction for overconvergent F-isocrystals, IV: Local semistable reduction at nonmonomial valuations, arXiv:0712.3400v3(2009).

[12] R. Kiehl, Die de Rham Kohomologie algebraischer Manningfaltigkeiten über einem bewerteten Körper, Pub. Math. IHES 33(1967), 5-20.

[13] A. Shiho, Crystalline Fundamental Groups I - Isocrystals on Log Crystalline Site and Log Convergent Site, J. Math. Sci. Univ. Tokyo, 7(2000), 509-656.

[14] A. Shiho, Crystalline fundamental groups II - Log convergent cohomology and rigid cohomology. J. Math. Sci. Univ. Tokyo, 9(2002), 1-163.

[15] A. Shiho, Relative log convergent cohomology and relative rigid cohomology I, arXiv:0707.1742v2(2008). 